\title{
Exploring Family-Centered Care Among Pediatric Oncology Nurses
}

\section{Lyndsay Jerusha MacKay}

Bachelor's of Nursing, University of Lethbridge, 2006

\author{
A Thesis \\ Submitted to the School of Graduate Studies \\ of the University of Lethbridge \\ in Partial Fulfilment of the \\ Requirements for the Degree

\section{MASTERS OF HEALTH SCIENCES (NURSING)}

\author{
Health Sciences \\ University of Lethbridge \\ LETHBRIDGE, ALBERTA, CANADA
}

C Lyndsay MacKay, 2009 


\section{Dedication}

To all of the children at the Alberta Children's Hospital who have fought cancer; those who have won and those who have lost.

To all of the pediatric oncology nurses at the Alberta Children's Hospital; for putting your heart and soul into caring for children with cancer and their families. 


\section{ABSTRACT: EXPLORING FAMILY-CENTERED CARE AMONG PEDIATRIC}

ONCOLOGY NURSES AT THE ALBERTA CHILDREN'S HOSPITAL

Family-centered care (FCC) is important within the practice of pediatric oncology nurses. Such nurses face challenges and barriers when attempting to provide FCC. The purpose of this study was to understand the experiences of pediatric oncology nurses in relation to FCC; identify how pediatric oncology nurses implemented FCC into their practice; identify what facilitated and enabled pediatric oncology nurses to implement FCC; and discern the barriers and challenges that were present in their setting when implementing FCC. A qualitative approach utilizing person-centered interviewing was used to collect data. Nurses ( $\mathrm{N}=20)$ from the Alberta Children's Hospital were recruited through purposeful convenience sampling and were then interviewed. Five major themes were identified from the data set: ACH support f FCC, How participants defined FCC, Establishing FCC, Enhancing FCC, and Barriers and Challenges to providing FCC. Recommendations for future research and implications for practice and education are offered. 


\section{Acknowledgments}

I would like to especially thank Dr. David Gregory for taking me under your supervision as a graduate student and investing much of your precious time on my work. You were extremely helpful as you taught me with enthusiasm and encouraged me along the way.

Thank-you to Dr. Ruth Grant-Kalischuk and Dr. Barry Hall for sitting on my committee and faithfully providing productive and insightful feedback that helped me to grow and mature at conducting qualitative research.

Thank-you to Dr. Sonya Grypma for graciously encouraging and inspiring me to pursue a master's degree. Thank-you to Catherine Lang for supporting my research thesis within the pediatric oncology program at the Alberta Children's Hospital (ACH). Thankyou to Dr. Aru Narendran for helping me to get this study through ethics. Thank-you to all of the pediatric oncology nurses at the $\mathrm{ACH}$ that took time out of their busy schedules to be part of this project.

Finally, I would like to thank my husband and mother for being there to support me along the way. 


\section{TABLE OF CONTENTS}

TITLE PAGE i

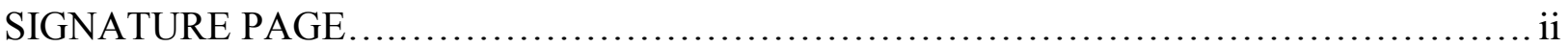

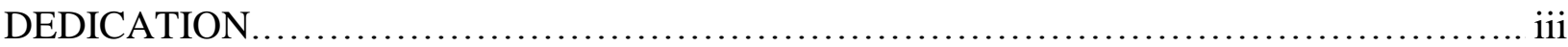

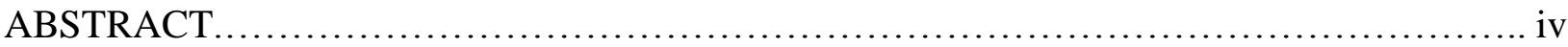

ACKNOWLEDGMENTS ................................................................

TABLE OF CONTENTS ................................................................. vi

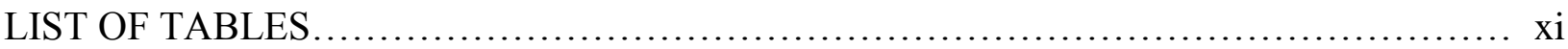

LIST OF CHARTS .....................................................................

CHAPTER ONE - STATEMENT OF THE PROBLEM

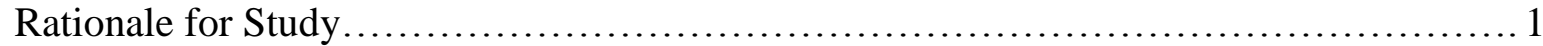

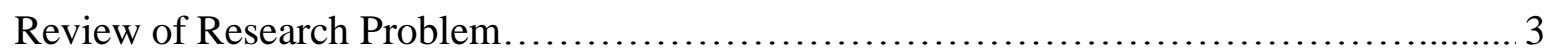

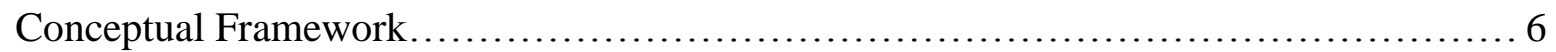

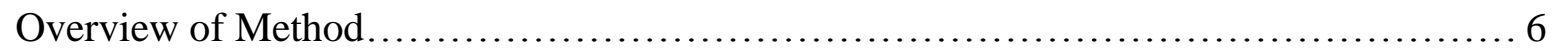

Problem Statement and Purpose...................................................... 8

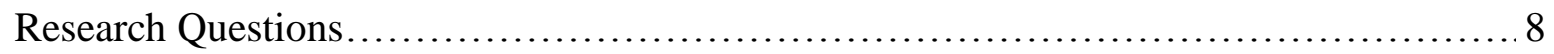

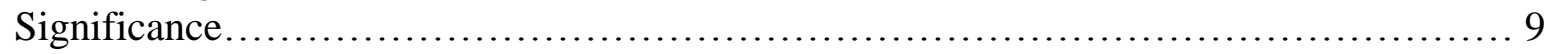

CHAPTER TWO - REVIEW OF THE LITERATURE

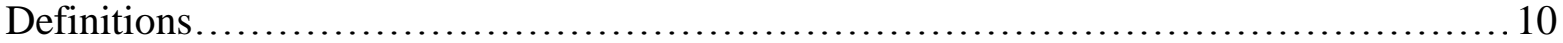

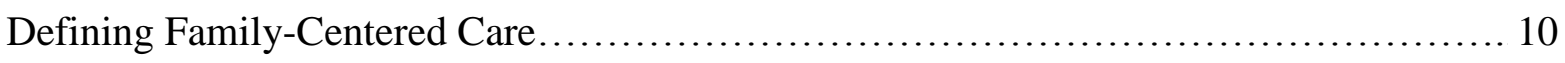

History of Family Centered Care in Pediatrics..................................... 13

Family-Centered Care in Pediatrics.................................................. 14

Parent as the Expert ..................................................................... 16

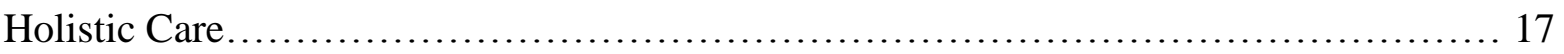

Listening and Communication.................................................... 17

Patients and Families Deserve Control.............................................. 18

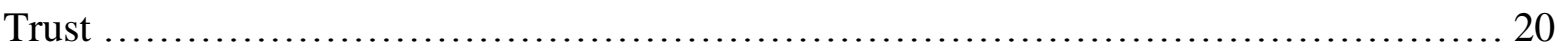

Importance/Significance of Family-Centered Care in Pediatrics......................... 20

Family-Centered Care as an Anti-Oppressive Practice..................................... 24

Parents Perception of Family-Centered Care ............................................. 25

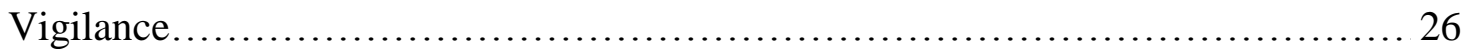

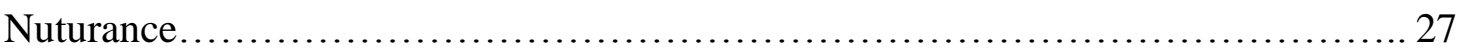

Understanding the Lack of Family-Centered Care in Pediatric Nursing: 29 
Challenges and Barriers.

Current Difficulty Implementing Family-Centered Care........................... 33

Lack of Institutional Support for Family-Centered Care.......................... 35

Pediatric Oncology Nursing and FCC ......................................... 37

Childhood Cancer Statistics................................................ 37

Stress from Childhood Cancer............................................. 38

Nursing Pediatric Oncology Patients........................................ 40

Family-Centered Care among Pediatric Oncology Nurses........................ 44

Summary of Literature ........................................................ 48

\section{CHAPTER THREE - RESEARCH DESIGN}

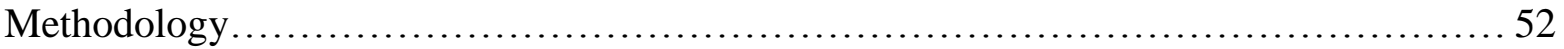

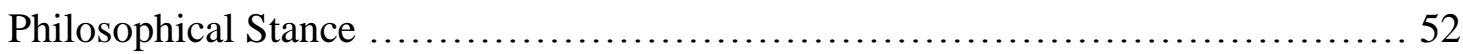

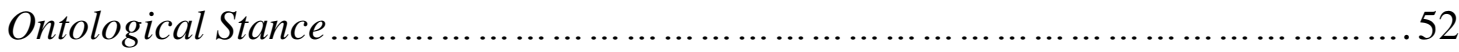

Epistemological Stance................................................... 53

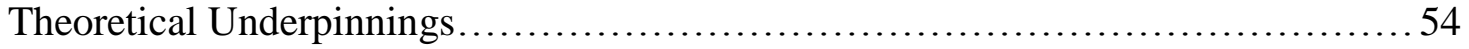

Researcher's Personal Perspective.............................................. 54

Method......................................................................... 55

Person Centered Interviewing ........................................... 56

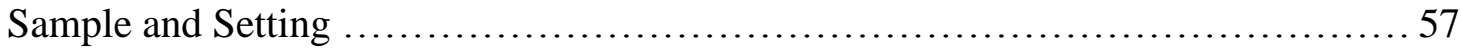

Access and Recruitment................................................. 58

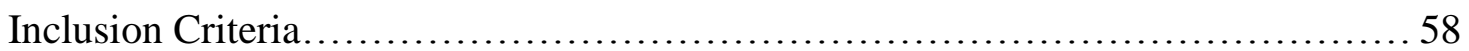

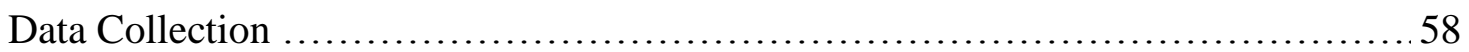

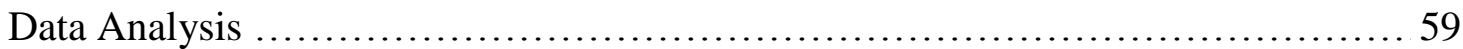

Ethical Approval Process.....................................................6 61

Ethical Issues............................................................ 62

Scientific Rigor .......................................................... 64

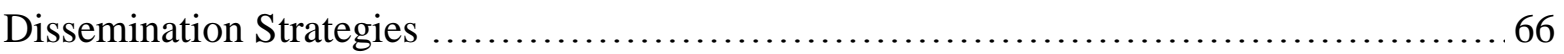

\section{CHAPTER FOUR - FINDINGS}

Does the ACH Support Family-Centered Care (FCC)?............................................. 71

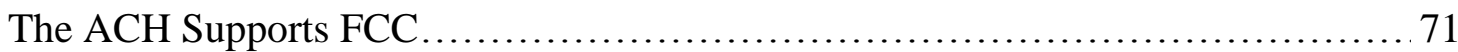

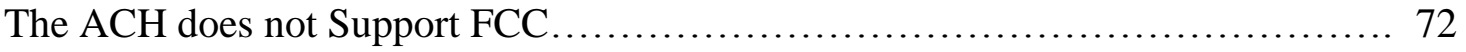

How Participants Defined FCC ............................................... 73

Encompassing the Whole Family ......................................... 74

The Family Identified who Constituted their Members........................ 74

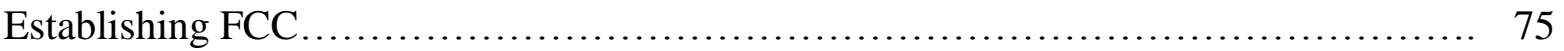

Collaborating with the Patient-Family: The Three Legged Race................. 75

Patient-Family are Part of the Care Team................................ 75

Offering Choices and Fostering Control.................................... 77

Parent as the Expert.................................................. 80 


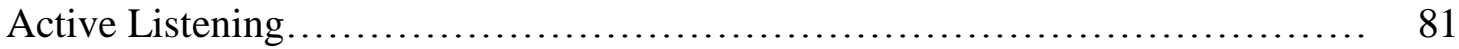

Respecting Culture and Beliefs........................................... 82

Not Judging the Patient-Family ........................................ 82

Learning about Specific Cultures and Beliefs........................... 84

Enhancing FCC. 86

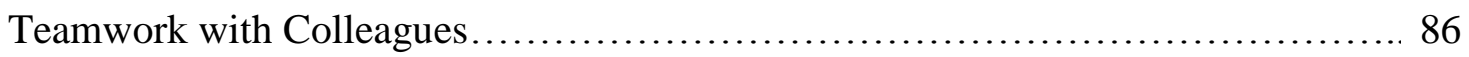

Importance of Teamwork with Colleagues.................................. 86

Social Work, Child Life, Physicians and Spiritual Care........................ 87

Nurse Patient-Family Relationship........................................... 89

Fostering Comfort and Tailoring Nursing Care ........................... 90

Small Things make a Big Difference.................................... 92

Nursing Presence and According Personhood to Patient-Families................ 93

View the Patient-Family as Human Beings.................................... 93

Outcomes Associated with the Nurse Patient-Family Relationship............. 96

Letting Parents Cope in their Own Way..................................... 96

Encouraging the Cancer Community......................................... 97

Having the Attitude that there is Always an Opportunity......................... 98

Being Flexible Rather than Dictating and Controlling............................. 99

Caring for the Parent.................................................... 100

Educating the Patient-Family ............................................. 102

Different Stages and Types of Learning ................................. 102

Education Enables and Empowers..................................... 103

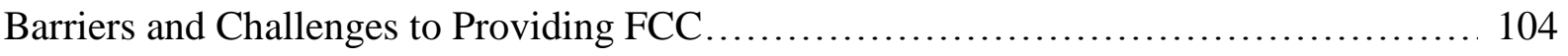

Not Understanding Patient and Family Culture........................................ 104

Fear of Precipitating a Negative Event or Outcome

when Lacking Cultural Knowledge............................................. 106

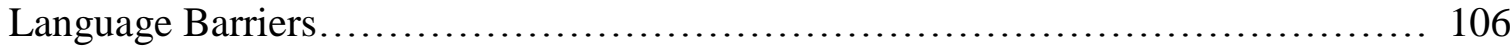

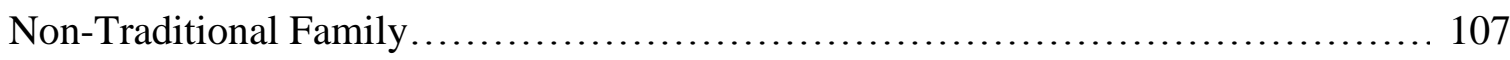

Contradictions and Tensions between Nurses and Families.......................... 108

Disagreeing with Decisions Made by Patient-Families......................... 109

Disagreement with Families' Spiritual and Religious Beliefs................... 112

Unable to Fulfill Parents' Requests and Wishes............................ 115

Lack of Time and Increasing Acuity of Patients................................ 116

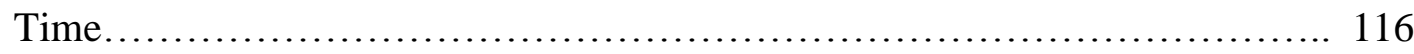

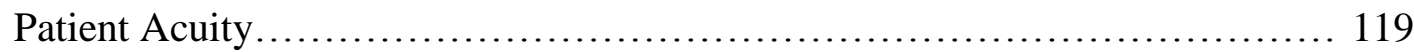

Inadequate Staffing ..................................................... 120

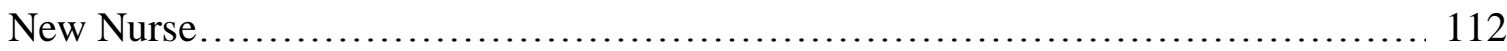

Budget and Resources......................................................... 122

Limited Space and Lack of Resources......................................... 123

Lack of Education about FCC for Nurses and Health Care Providers................ 125

Difficult Working Relationships............................................. 126 
Non Trusting Families.................................................. 126

Difficult Families...................................................... 128

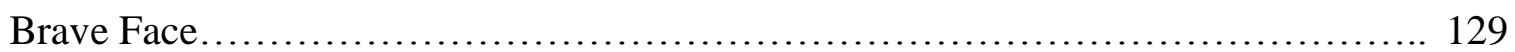

Not Passing Along Information............................................. 130

From Nurse to Nurse ............................................... 130

From Health Care Professional to Patient-Family ........................ 131

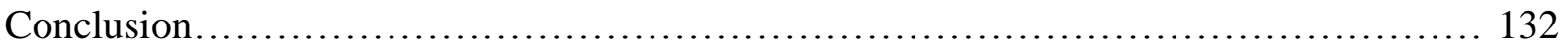

\section{CHAPTER FIVE - DISCUSSION}

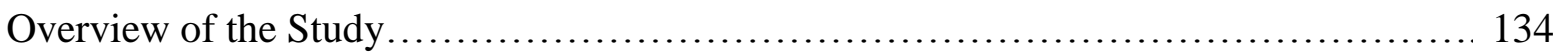

Usefulness of the Theoretical Framework....................................... 137

Implementation of FCC; Strategies and Barriers................................ 138

FCC from a Systems Based Perspective ............................................. 139

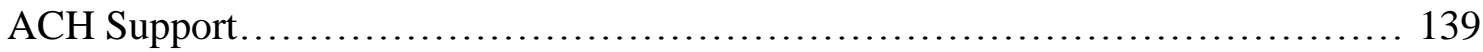

Involving Parents in Rounds.............................................. 140

Interdisciplinary Approach................................................ 141

Nursing Care Perspective ........................................................ 143

Mentoring the New Nurse ................................................ 144

Nurse and Patient-Family Perspective ........................................... 146

Not Discussing the Dying Child or the Word Cancer.............................. 146

Moral Distress.......................................................... 147

Care for Whole Family in Contest of the Patient Family.......................... 149

Nursing Presence........................................................ 150

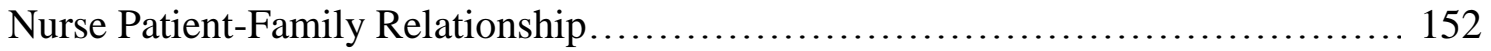

Cultural Diversity of the Patient-Family..................................... 155

Educating the Patient-Family............................................ 157

Barriers at all Levels.............................................................. 158

Is FCC the Solutions? The Ideal, Reality and Making Sense of it all.................. 160

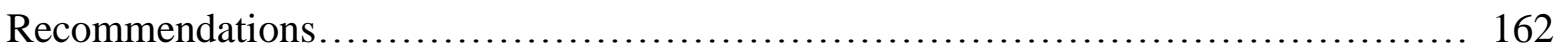

Practice Administrative Recommendations......................................... 164

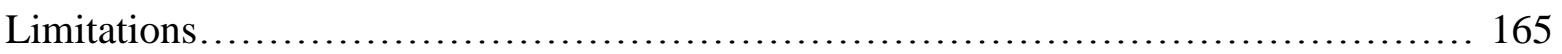

Future Research.................................................................... 166

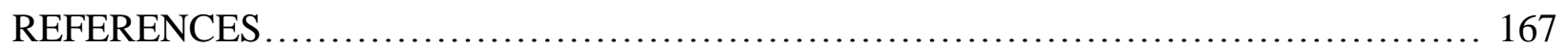

\section{APPENDICES}

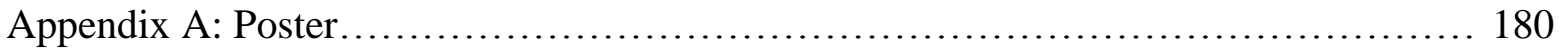

Appendix B: Information Sheet............................................... 181

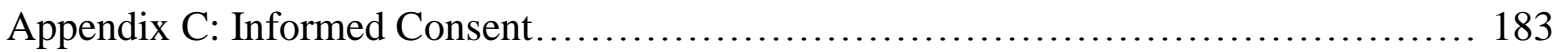

Appendix D: Demographic Questionnaire ...................................... 187

Appendix E: Interview Guideline............................................... 188 


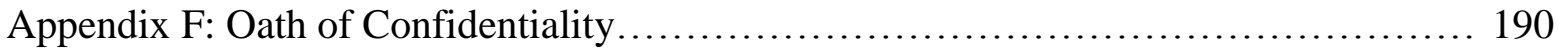

Appendix G: Timeline and Budget............................................. 191 


\section{LIST OF TABLES}

Table 4.1 Major themes Extracted from Data....................................68

Table 4.2 Demographic Information of Participants............................ 70

Table 5.0 Strategies and Barriers to FCC Among Pediatric Oncology Nurses.........139 


\section{LIST OF CHARTS}

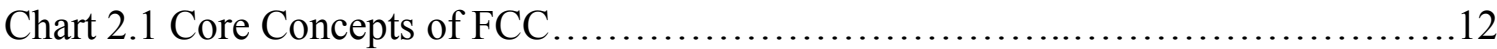

Chart 2.2 Core Principles of FCC in Pediatrics................................... 15

Chart 2.3 Summary of the Literature .................................................. 


\section{CHAPTER ONE: STATEMENT OF THE PROBLEM}

Introduction: Rationale for Study

Family-Centered Care (FCC) is recognized at the Alberta Children's Hospital $(\mathrm{ACH})$ as an important part of care provided to children and their families. Childhood cancer is very complex and requires nurses to perform technical procedures and provide complicated treatments to patients. Pediatric oncology nurses face many challenges and barriers in attempting to implement FCC within their practice. Unfortunately, there is limited information in the nursing literature that gives pediatric oncology nurses direction and guidance on providing FCC.

There is currently no formal FCC education or training that is accessible to nurses at the $\mathrm{ACH}$. The $\mathrm{ACH}$ does not hold education sessions on $\mathrm{FCC}$ or make the philosophy of FCC readily available for nurses to learn. As well, there is no working policy of FCC that provides nurses with guidance, knowledge and information on how to implement FCC into their practice and make it a standard of care for patients and their families. This is of concern because nurses are the front line staff who have direct and frequent contact with patients and their families.

The Decision and Research Support Team (DSRT) distributed the "Patient Family Feedback Form" questionnaire to 171 parents and guardians of patients from the inpatient oncology unit at the ACH between September 2006 and September 2007 (Chan \& Kennedy, 2008). In the questionnaire, respondents, who had their child stay on the inpatient oncology unit, were asked to choose an aspect of FCC that they felt needed improvement. The following three aspects of FCC were found to need improvement: helping you to be an active part of getting the desired resources and support; helping you 
learn about and understand what was happening to your child; and responding to your concerns and observations.

Respondents were also asked to indicate the level of agreement with their inpatient experience in the following areas: medication, information, access to nurses, navigating the hospital, and discharge planning (Chan \& Kennedy, 2003). Access to nurses received the lowest overall score. The following negative comments were made regarding their inpatient experience: "some nurses/doctors... I'm sure they're tired or stressed due to so many ill kids to care for," "Overworked? Short staffed? I think so," "I wish people who cared for my child had listened to my concerns... I felt that they had placed too much emphasis on my child's quantitative findings rather than her clinical signs and symptoms, which greatly increased and prolonged my child's suffering to the point of her nearly dying," "More nurses! I feel they work too hard and can become quite tired," and "Of course we need more nursing staff but that can't be helped."

As a result of these findings, the DSRT recommended implementing quality improvement initiatives to foster the successful implementation of FCC. This study has occurred at a pivotal time point within the oncology department at the $\mathrm{ACH}$ because it can help inform the quality improvement initiative that addresses FCC among the nurses.

According to Shields (2007) "health care practitioners must critically examine family-centered care and how it is delivered" (p. 869). Baker (1995) suggests that pediatric nurses need to attain particular skills, knowledge and experience to implement family-centered care into their practice with children. Such information does not appear to have been given consideration from nursing leaders, such as hospital administration 
and nurse managers (Baker, 1995). There is a lack of understanding on how to successfully deliver FCC and on the knowledge of nurses' experiences.

The ACH recognizes that FCC is important in caring for children and their families. This is confirmed by their new facility which is designed based on FCC and their Family-Resource Centre.

\section{Review of the Research Problem}

FCC has become a term that is used widely among pediatric health care worldwide (Shields \& Tanner, 2004). FCC is increasingly perceived to be essential in the delivery of care to children and their families (Regan, Curtin \& Vorderer, 2006). Family members have the greatest influence and impact on a child's life and they are the experts about the care that their children require during their hospital stay (Institute for FamilyCentered Care, n.d.).

At the core of FCC in pediatrics is the understanding that the child's family is the primary source of strength and support for him/her (American Academy of Pediatrics, 2003). The family is a central part of the environment in which children grow, develop and function. Families play a critical role in the development and nurturing of children (Denboba et al., 2006). Thus, health care professionals are encouraged to understand and work within the context of the family in order to have a positive impact on children's health and well-being (Begun, 1996).

"Pediatric oncology nursing is based on family-centered care" (Ogle, 2006, p. 29). The children and their families become the heart of pediatric oncology nursing because children live within the context of their families (Ogle, 2006). Nurses are wise to involve 
the family in the care provided for children with cancer because the family is the constant support that enables children to endure their cancer treatment journey.

There have been many advances in treatment outcomes and survival rates among children with cancer, which have influenced the objectives and goals of nurses caring for such children (Cantrell, 2007). Currently, pediatric oncology nursing care focuses on implementing clinical trial protocols, educating families about the short term and long term side effects of chemotherapy and radiation, and providing optimal psychosocial supportive care during treatment as a means to enhance patient's quality of life during their therapy and survivorship (Cantrell, 2007). This means that "the nature of care provided in inpatient, outpatient and community settings continues to change, with clinical advances, and children with cancer requiring highly specialized care" (Tomlinson, 2004, p. 646). Such advancements make it more difficult and complex for nurses to care for children with cancer and their families.

In the midst of such complex care environments, it is important that pediatric oncology nurses do not become task orientated and lose sight of the holistic and human aspects of nursing practice. "If the artistic nature and caring presence are stripped from the practice of pediatric oncology, patients and families will surely suffer, and positive treatment outcomes will decline" (Cantrell, 2007, p. 137). FCC is a method of care delivery that encourages pediatric oncology nurses to focus on the humanist aspect of their patients, and foster the art of nursing.

Schwartz, A 40-year-old health care lawyer with cancer, publicly expressed gratitude to his nurses for "moments of exquisite compassion" and an "extraordinary array of human and humane responses" (1995, p.1). To Schwartz (1995), "quiet acts of 
humanity have felt more healing than the high-dose radiation and chemotherapy that hold the hope for cure" (p.2). As a result of such acts, Schwartz founded a center that is dedicated to strengthening the relationship between the patient and caregiver. From his perspective, it is the relationship between the nurse and patient that truly fosters holistic healing for patients as they undergo their treatment journey. FCC is the vehicle that nurses can use as a means to create such relationships with patients and families.

Unfortunately, FCC does not exist in all health care settings, including pediatrics (Hemmelgarn, Glisson \& Dukes, 2001). Not all pediatric nurses incorporate FCC into their practice. According to Bruce and Ritchie (1997), researchers have found that "nurses do not view the needs of the family as their responsibility," and may lack the skills and knowledge to provide FCC (p. 214). As a result, parents often believe health care professionals lack respect for and recognition of their expertise as caregivers (Bruce and Ritchie, 1997).

FCC lacks in clarity in both the literature and practice contexts (MacKean, Thurston \& Scott, 2005). There are various definitions and theories of what FCC entails, but little information provides practical guidance and direction for nurses. Upon conducting the literature review for this research study, there was a lack of concrete examples and ways to implement FCC into nursing practice. Also, there was a lack of evidence and information on FCC in the pediatric oncology setting. According to Galvin et al. (2000), there is often limited support, from an organizational perspective, to implement FCC into nursing practice. In order for FCC to be successfully incorporated into practice, the institution in which health-care professionals work must actively support and encourage such practices. 
A qualitative study about the conceptualization of FCC was conducted by MacKean, Thurston and Scott (2005) at the Alberta Children's Hospital. They found that health-care providers recognized the current health care system was not user-friendly, and parents and health-care providers felt that there was a need for system change. Opportunities for change included: help with advocacy and care coordination, information about services, coordination of service transition, coordination within and between services, increase resources and services that are truly driven by family needs. Thus, there is a need to explore FCC among pediatric oncology nurses at the ACH.

This study was necessary and relevant because pediatric oncology nursing is a complex specialty that comes with a challenging environment in which to incorporate FCC. No concrete examples about how to implement FCC were located in the literature. There was also limited information in the nursing literature concerning pediatric oncology nurses and FCC. This is unfortunate because pediatric oncology nurses care for children with chronic and sometimes fatal diseases in which the family is extremely involved. Therefore, conducting this study was justified given the limited knowledge about FCC and pediatric oncology nursing.

\section{Conceptual Framework}

The conceptual framework used to guide this study arose from the Institute of Family-Centered Care. This framework directed the development of the semi-structured interview guide that was used for this study. According to the Institute of FamilyCentered Care, FCC is an innovative approach to the planning, delivery, and evaluation of health care, and is grounded in the mutually beneficial partnership among health care providers, patients and families (The Institute for Family-Centered Care, n.d.). This 
framework consists of four core concepts; dignity and respect, information sharing, participation, and collaboration (The Institute for Family-Centered Care, 2008). Dignity and respect directs health care practitioners to listen to their patient and family's choices and perspectives regarding their healthcare, and to act on them accordingly. Also, the patient and his/her family's knowledge, beliefs, values, and cultural backgrounds are integrated into the planning and delivery of care. Information sharing requires health care practitioners to communicate and share unbiased and complete information with patients and their families. Such information is provided in a timely, complete and accurate manner so that patient and their families are able to actively engage in care and decisionmaking. Participation includes encouraging patients and families to engage in decisionmaking and care to the extent to which they choose. Collaboration involves health care leaders consulting with patients and their families to develop policies and programs, implementation and evaluation of care, and professional education.

\section{Overview of Method}

The research methodology chosen for this study was qualitative, the research method entailed person-centered interviewing (Levy \& Hollan, 1998). In this study, 20 pediatric nurses from the oncology department at the $\mathrm{ACH}$ were interviewed for approximately one hour regarding their perspectives of FCC, skills and approaches they used to provide such care, and barriers and challenges to FCC. Person-centered interviewing is used to gain an understanding that goes beyond role-determined surface scripts, and to reveal underlying elements of the organization of persons, the sociocultural environment, and their interactions among one another (Levy \& Hollan, 1998). 
In this study, the interviews were transcribed verbatim and read over multiple times. Thematic analysis was used to discover over-arching themes (Polit \& Hungler, 1999). Such themes described and offered insight into the phenomenon of FCC among pediatric oncology nurses at the $\mathrm{ACH}$. A better understanding into $\mathrm{FCC}$ among oncology pediatric nurses at the $\mathrm{ACH}$ was obtained from this study.

\section{Problem Statement and Purpose}

Although FCC has been recognized as fundamental to pediatric oncology nursing care at the $\mathrm{ACH}$, barriers hinder it from being practiced by all nurses and successfully implemented as intended. To date, research examining FCC within the specialty of pediatric oncology nursing remains limited.

The purpose of this study was to understand the experiences and nature of FCC among pediatric oncology nurses, identify how pediatric oncology nurses implement FCC into their practice, identify what facilitated and enabled pediatric oncology nurses to implement FCC into their practice, and discern the barriers and challenges that were present in their setting when implementing FCC.

\section{Research Questions}

The research questions that guided this study were:

1) To what extent do pediatric oncology nurses at the $\mathrm{ACH}$ report practicing within the FCC framework?

2) What are the barriers and challenges faced by pediatric oncology nurses at the ACH when providing FCC?

3) What practical skills and approaches are used by pediatric oncology nurses to provide FCC in the midst of barriers and challenges? 
4) What strengths and capacities are associated with FCC that aid pediatric oncology nurses in providing care to children with cancer and their families?

5) How does the ACH support and encourage the implementation of FCC by nurses within the oncology program?

\section{Significance}

The results of this study will ultimately benefit pediatric oncology nurses, managers, patients and their family members within the oncology program at the $\mathrm{ACH}$ as a greater understanding of FCC is gained. The practical skills and approaches pediatric oncology nurses used to implement such care were identified in this study and they will be shared with the pediatric oncology nursing environment. Barriers and challenges faced by pediatric oncology nurses as they implemented FCC into their practice were also identified. The strengths and benefits of FCC among pediatric oncology patients were established. The findings also have implications regarding the potential creation of a FCC policy. 


\section{CHAPTER TWO: REVIEW OF THE LITERATURE}

Family-centered care (FCC) has become an increasingly important topic among the profession of nursing. In the following section, FCC will be defined and explained. More specifically, FCC will be defined and explained from a pediatric perspective. Following these definitions, a brief history of FCC in relation to pediatrics will be provided. At the end of this section, FCC will be discussed from the perspective of pediatric oncology nursing; caring for children with cancer and their families. A chart at the end of this chapter summarizes the seven major studies that form the majority of this literature review (see Chart 2.1). The studies included in this chart provide an overview of the nature of research conducted regarding FCC up to date.

\section{Definitions}

For the purpose of ensuring clarity of key concepts used throughout this thesis, the following definitions are provided. Family is "two or more persons who are joined together by bonds of sharing and emotional closeness and who identify themselves as being part of a family" (Friedman, Bowden \& Jones, 2003, p. 651). Family nursing is "the provision of care to families and family members in health and illness situations" (Friedman, Bowden \& Jones, 2003, p. 651). Pediatric oncology nursing is planning and providing nursing care to children, from birth -18 , with cancer that involves their physical, emotional, mental and spiritual well-being.

\section{Defining Family-Centered Care}

FCC, its philosophy, definition, principles and benefits have been studied extensively since the 1970's (Peterson, Cohen \& Parsons, 2004). Fields such as 
psychology, education, social work, medicine, nursing, and allied health have recognized the significance of working with families in caring for patients (Begun, 1996).

FCC is an innovative approach to the planning, delivery, and evaluation of health care, and is grounded in the mutually beneficial partnership among health care providers, patients and families (The Institute for Family-Centered Care, n.d.). The core concepts of FCC include dignity and respect, information sharing, participation, and collaboration (The Institute for Family-Centered Care, 2008). Dignity and respect requires that health care practitioners listen to the patient and family's choices and perspectives regarding their healthcare and act on them accordingly. The knowledge, beliefs, values, and cultural backgrounds of the patient and family are integrated into the planning and delivery of care. Information sharing entails health care practitioners to communicate and share unbiased and complete information with patients and families. Such information is provided in a timely, thorough and accurate manner so that patients and families are able to actively participate in care and decision-making. Participation includes encouraging patients and families to participate in care and decision-making to the extent in which they choose. Collaboration requires the administrators of hospitals to consult with patients and families in the development of policies and programs, implementation and evaluation of care, and the creation of professional in-service education. 


\section{Chart 2.1}

\section{Core Concepts of FCC}

1. Dignity and Respect - Health care practitioners listen to and honor patient and family perspectives and choices. Patient and family knowledge, values, beliefs and cultural backgrounds are incorporated into planning and delivery of care.

2. Information Sharing - Health care practitioners communicate and share complete and unbiased information with patients and families in ways that are affirming and useful. Information is timely, complete and accurate.

3. Participation - Patients and families are encouraged and supported in participating in care and decision-making at any level they choose.

4. Collaboration - Patients, families, health care practitioners and hospital leaders collaborate in policy and program development, implementation, and evaluation; in health care facility design; and in professional education, as well as in the delivery of care.

Institute for Family-Centered Care (2008)

Originally, nursing practice was founded upon the traditional medical model of care that emphasized restricting patients and families (Institute for Family Centered Care, n.d.). Patients and families were expected to act in accordance to the wishes of the healthcare professionals (Institute for Family-Centered n.d.). In contrast, the familycentered model recognizes the strengths that families and patients can bring to the healing process and promotes the incorporation of them in a collaborative manner (Institute for Family-Centered Care, n.d.).

FCC is based on the following assumptions: (1) people are basically good, (2) people have strengths, (3) people have skills, abilities and knowledge, (4) people need support and encouragement, (5) families have hopes, dreams and wishes for their children, (6) families are resourceful, but all do not necessarily have access to recourses, (7) families should be assisted in ways that help them maintain their dignity and hope, and (8) 
families should be equal partners in the relationship with service providers (Keyser, 2006).

After exploring the definition and description of FCC it becomes apparent why it is important to incorporate it in the care provided to children. This is because children rely heavily on their parents and family members because adults are their guardian and primary caregiver. The following section will explore FCC among pediatrics.

\section{History of Family-Centered Care in Pediatrics}

The health of children became a concern to society in the 1880 's because of urbanization, industrialization, and massive immigration (Deatrick, 2006). At the same time, there was an increase in recognition of death and suffering among high-risk infants and children from infectious diseases. Such concern and recognition influenced a movement to enhance the health and well-being of infants and children (Deatrick, 2006). As a result, hospitals and centers were created to care for ill infants and children.

From 1920 and to the end of World War II, nursing was characterized by the battle of science against infectious disease (Jolley \& Shields, 2009). Antibiotics were not present, thus health professionals were in a battle against the spread of infections; priority was not given to the emotional needs of the child (Jolley \& Shields, 2009).

Up until the 1950's, parents were viewed as a negative factor in the care of hospitalized children (Jonkees, 1955). In the 1950's and 1960's there were restrictive visiting policies in place; parents were only allowed to see their children for a couple of hours each day (MacKean, Thurston \& Scott, 2005). Health-care providers discouraged the involvement of families, and boldly told them what to do (MacKean, Thurston \& Scott, 2005). 
Jolley (2004) examined the history of children's nursing within the hospital setting in the UK between 1920 and 1970. She found that the patients were traumatized when hospitalized because the staff separated them from their parents for extended periods of time. Also, the staff that cared for the patients were construed as being nonhuman and lacked affection.

World War II caused an increase in the recognition of psychological health issues among adults and children (Jolley \& Sheilds, 2009). As a result, it was acknowledged that it was stressful for children to be in a hospital and separated from their parents (Paliadelis et al., 2005). The idea of including parents in children's care was introduced by James Robertson (Alsop-Shields \& Mohay, 2001). James Robertson used evidence collected by John Bowlby, an internationally recognized theorist, to demonstrate the great distress children experienced when separated from their parents (Alsop-Shields \& Mohay, 2001). James Robertson used such information to campaign and form strong vocal and lobby groups to improve the conditions for children in hospitals. Their work influenced and facilitated change to hospital policies that improved the care provided to children in countries such as: Canada, USA, Britian, Australia, and European nations (Alsop-Shields \& Mohay, 2001). As a result, families were included in the care of children during hospital stays and FCC was introduced to health care systems worldwide.

\section{Family-Centered Care in Pediatrics}

FCC has become a term that is used widely among pediatric health care worldwide (Shields \& Tanner, 2004), and is perceived to be essential in the delivery of care to children (Regan, Curtin \& Vorderer, 2006). This is because family members have the greatest influence and impact on a child's life, and families are the experts about the 
care that their children require during their hospital stay and at home (Institute for Family-Centered Care, 2008).

The practice of pediatrics is unique among medical specialties in many ways, among which is the nearly certain presence of a parent when health care services are provided for the patient. Regardless of whether parents or other family members are physically present, their influence is pervasive. Families are the most central and enduring influence on children's lives. (American Academy of Pediatrics, 2003, p. 1541)

At the core of FCC in pediatrics is the understanding that the child's family is the primary source of strength and support for him/her (American Academy of Pediatrics, 2003). Families play a critical role in the development and nurturing of their children (Denboba, McPherson, Kenney, Strickland, \& Newacheck, 2006). The family is the environment in which children grow, develop and function. Thus, health care professionals must understand and work within the context of the family in order effectively support children (Begun, 1996). See Chart 2.2 below for the summary of the importance of FCC among pediatrics.

\section{Chart 2.2}

Core Principles of FCC in Pediatrics

1. Families and professionals work together in the best interest of the child and family

2. Everyone's skills and expertise are bought into the relationships

3. Trust is fundamental

4. Communication and information sharing is open and objective

5. There is a willingness to negotiate

Denboba et al. 


\section{Parents as the Expert}

Parents are the constant in a child's life. They are with the child on a continuous basis, through a variety of settings, and through the different developmental stages; therefore, they are the 'experts' (Shelton \& Stepanek, 1994). In order for pediatric care to be family-centered, parents' choices, recommendations, and observations must be taken seriously (Shelton \& Stepanek, 1994).

In pediatrics, FCC acknowledges that the child's family is pivotal in the care provided by health care professionals (Bruce \& Ritchie, 1997). The family is the professional and has a say in designing how health services are to be delivered (Shelton \& Stepanek, 1994). FCC within the pediatric specialty entails that the health care professionals focus their efforts on developing supports and resources for children and their families that will be continued when health care professionals are absent (Shelton \& Stepanek, 1994). Health care professionals are not present when the child goes home, therefore, it is important to ensure the family has the required supports and are competent with respect to the care of their child. FCC depends on true partnership, where the health care professionals and family members "are of mutual benefit to each other as an integral members of the child's care team through the sharing of information from their unique perspectives" (Shelton \& Stepanek, 1994, p. 7).

Within FCC, the family is the constant in the child's life and the expert on the well-being and needs of the child (Allen \& Petr, 1996). Parents should be partners with health-care professionals in the planning of care for children (Allen \& Petr, 1996). FCC includes moving from paternalism to enablement and from a judgmental to a supportive stance (Epps \& Jackson, 2000). The ultimate goal of FCC within pediatrics is to be able 
to discharge a child into a family that is empowered and able to continue promoting and nurturing the child's health and well-being when at home.

\section{Holistic Care}

The nursing profession prides itself in that it endeavors to provide care that is holistic; care that meets the physical, emotional, mental and spiritual needs of a person. "Family-centered care is a holistic approach to working with children and their families" (Keyser, 2006, p. 12), because it encourages nurses to create a relationship with the patient and family, not merely conduct technical tasks. It is the means in which nurses can provide care that meets the physical, emotional, mental and spiritual needs of children.

\section{Listening and Communication}

Listening is a key component in FCC among pediatric nursing. According to Shelton and Stepanek (1994), "listening to and understanding how the family defines itself is the first, and most critical, step to providing family-centered care" (p. 6). If nurses define the families they care for based on stereotypes or their own limited experience, then their ability to implement FCC is jeopardized (Shelton \& Stepanek, 1994). Within the FCC framework nurses are attentive and give children and their family member's opportunities to voice their opinions, beliefs, values and expectations. Nurses also incorporate such information into the planning and delivery of care.

The provision of family-centered care requires nurses to recognize the importance of communications in developing trusting relationships with patients and their families (Regan, Curtin \& Vorderer, 2006). According to Yingling (2000), nurses are providers of information and emotional support, and they have the ability to explain medical 
procedures in ways that children and family members can understand. Within FCC, nurses are aware of their communication method when providing care to children and their families because communication can either ease and comfort the patient and their family or create stress and uneasiness.

\section{Patients and Families Deserve Control}

When practicing within the FCC framework nurses seek to empower children in ways that are appropriate for their age and development. The child's right to exercise choice is inexorably linked to FCC as it encourages them to be partners in their care and enhances their control and independence (Regan, Curtin \& Vorderer, 2006). This can be accomplished by giving the patients the ability to choose and control their own care while in the hospital. When nurses provide FCC they have the ability to prevent children from becoming dependent on health-care professionals and empower them to make decisions regarding their own health and well-being.

If critical choices are to be made, FCC demands that families are involved in the decision making process (Shelton \& Stepanek, 1994). Health care professionals must provide information and create opportunities for choices to be made by patients and their families (Shelton \& Stepanek, 1994). Thus, nurses who practice the FCC framework collaborate with family members and provide opportunities for families to voice their suggestions and wishes in the decision making process. As a result, care decisions will be tailored to the needs of the individual child and their families, and an optimal caring environment will be created.

Shields, Pratt and Hunter (2006) define FCC as "a way of caring for children and their families within health services which ensures that care is planned around the whole 
family, not just the individual child/person, and in which all the family members are recognized as care recipients" (p. 1318). Family members are affected by the child's illness and hospitalization because it is a stressful event that has an impact on their daily routine and can be emotionally draining. Therefore, the family members must be an integral part in the care children receive.

FCC involves creating collaborative relationships with patients and families. It does not include teaching families how to care for their children, then leaving the families with the entire responsibility (MacKean, Thurston \& Scott, 2005). Fundamental aspects of FCC include empowerment-enhancing methods and participatory components (Dunst, Trivette \& Deal, 1994). A relational component is central to family-centered care and is effectively demonstrated by respectful communication, positive professional attitudes about family competencies and wisdom (Dunst, Trivette \& Deal, 1994). Therefore, FCC involves nurses creating a trusting relationship with the patient and their family as a means to empower them to actively participate in their care. As a result of a collaborative teamwork effort, the health and well-being of the child are enhanced.

Within the FCC model, the role of family members extends beyond providing care for the child. The various roles of family members include: peer support to other families, advocacy for one's own children, membership on hospital committees, involvement in systems change in health care, and education of clinicians (Dokken \& Ahmann, 2006). Family members are useful resources in helping other families cope and understand the illness process children. They are also important sources of information when creating programs and developing policies within pediatric hospitals. As well, 
family members are valuable in educating health care professionals on ways to provide FCC effectively.

\section{Trust}

Research has shown that trust is a key characteristic in FCC (Deatrick, 2006). "Family-centered care cannot be accomplished without mutual respect and trust on the level of the providers and families" (Deatrick, 2006, p. 46). Such trust should be established regardless of the patient's condition, situation of care, and setting (Deatrick, 2006).

Bricher (1999) found that developing trust is inherent in the relationship between the nurse and child, as well, the nurse and parents. Such trust can be challenged or lost during frightening or painful experiences (Bricher, 1999). Ways in which nurses can establish trust include: providing honest explanations, conveying a sense of genuine respect and like for the child, getting to know the child as a unique person, sharing one's personal self with the patient and family, not avoiding emotional detachment as a way to protect oneself, and recognizing that it is essential to incorporate physical touch, such as hugs and cuddles, into practice (Bricher, 1999). When providing FCC, it is important that nurses use such techniques in developing a trusting relationship with the child and family members.

\section{Importance/Significance of Family-Centered Care in Pediatrics}

There are an increasing number of chronically ill children; thus there is an increase in the number of families assuming the care of such children (Bruce \& Ritchie, 1997). This is placing demands on health care systems to find new approaches to meet such needs (Bruce \& Ritchie, 1997). As a result, FCC has received more attention and 
significance as a way of addressing the increase in number of chronically ill children. Also, health care professionals are realizing the importance and significance of including families in the planning, implementation and evaluation of care for children.

When health care professionals incorporate FCC into their practice they are more likely to meet the needs of families. This is important because if the family's needs are not met and the family is not involved in the care provided to their child, the child has an increase chance of suffering and the hospital stay can become longer and more challenging (Ryan \& Steinmiller, 2004). Therefore, FCC is important in caring for children because it can actually decrease a child's suffering; which is the ultimate goal of pediatric nursing.

FCC improves the satisfaction among patients and their families with the hospitalization experience (Van Riper, 2001). It helps to inform and involve parents in the care provided to their children, thus empowering them to become confident and competent when participating in care (Van Riper, 2001). As a result of FCC, parents are more satisfied with the care their children receive because they are able to be part of the health care team.

FCC involves open communication between nursing staff and family members. It helps parents to feel comfortable voicing their opinions, concerns and expectations. When parents' expectations are not met, feelings of anger can erupt; such feelings can be upsetting for the nursing staff (Griffin, 2003). As a result, nurses can actually begin to feel that their care is not valued or appreciated, thus they experience feelings of anger and become defensive (Griffin, 2003). A family-centered philosophy of care provides 
guidance for health care professionals on how to reduce conflict in such situations (Griffin, 2003).

The philosophy of FCC encourages nurses to provide care that involves emotional aspects of patients and their families. As shown in research, children are able to recover faster and parents are increasingly cooperative when emotional support is provided to parents during their child's hospital stay (Pratt \& Chitakis, 1998). Thus, family-centered principles help children recover faster because the emotional aspects of children and their family are tended to and cared for.

"Pediatric emergencies create apprehension and anxiety for both children and parents that can threaten the psychosocial functioning and well-being of the family" (Hemmelgarn, Glisson \& Dukes, 2001, p. 94). The emotional well-being of the family is affected by the response of the health care providers during such periods of stress (Hemmelgarn, Glisson \& Dukes, 2001). Thus, it is important for nurses to be educated and enabled to provide care that is sensitive to the emotions of patients and family members: FCC enables nurses to do just that.

Coyne (2006) conducted a grounded theory study to gain knowledge and insight into children's experiences of being hospitalized. Eleven children from ages seven to fourteen were interviewed, and the data was used to create four main categories. They include: separation from family and friends, receiving treatments and investigations, being in an unfamiliar environment, and loss of self-determination. These findings confirm that children can experience great distress during their hospital stay and require nurses to take steps to reduce such distress. FCC is a vehicle that enables nurses to provide care that meets the children and their families needs, thus reducing distress. 
"Patient and family-centered care has been linked to effective team performance, which can, in turn, have a positive impact on quality" (Ponte et. al, 2004, p.212). In the past, health-care professionals controlled the care decision for patients and did not include families in collaborative team efforts. When following the principles of FCC, such as including family members in the health care team, the risk of conducting medical errors decreases and patient safety is maximized (Ponte et. al, 2004).

Lundqvist and Nilstun (2007) conducted a qualitative study using open nonparticipation observation to elucidate if dignity remains intact for the family members of children during their hospital stay. Their findings indicate that dignity remains intact when FCC is provided where all parties encourage each other in a collaborative relationship. When practitioners care from their own perspective and do not see the individual in front of them, dignity is undermined (Lundqvist \& Nilstun, 2007). Therefore, when FCC is used in nursing practice the dignity of children and their families remains intact.

In summary, FCC is important to nursing practice for the following reasons:

- decreases the suffering experienced by children

- decreases the length of hospital stay

- decrease distress experienced by children

- decreases family-professional conflict

- increases care that is emotionally sensitive

- decreases errors and increase patient safety, and

- ensure the patient and their family dignity is intact. 


\section{Family-Centered Care as an Anti-Oppressive Practice}

In order for "nurses to act as empowerers, a new approach must be adopted" other than the traditional medical model to guide their practice (Martin \& Younger, 2000, p. 60). The medical model has previously dominated the way nurses practice and provide care to patients and their families. An anti-oppressive approach should be utilized by nurses that overrides aspects of rigid professionalism and paternalism, which is the basis of the medical model (Martin \& Younger, 2000).

Anti-oppressive practice involves equity, inclusion, transformation and social justice (Campbell, 2003). It considers the nurse and patient as equal partners who both possess valuable knowledge (Campbell, 2003). It entails the nurse to include the patient in decisions about care and treatment options. According to Hopton (1997), antioppressive practice seeks to overcome inequalities in power and encourage partnership in work. Anti-oppressive practice places the nurse and patient at the same level and seeks to empower patients and their families.

Nurses need to overcome disempowering situations in spite of the pressures imposed upon them by the traditional definition of their roles (Martin \& Younger, 2000). The medical model is a disempowering culture that is task orientated, a paternalistic approach to care, and remains caught in a rigid hierarchical structure (Martin \& Younger, 2000). There is a strict hierarchy between the 'expert professional self' of those who have clinical expertise and the patient and family (Hart \& Freeman, 2004). In this model, the nurse is the expert who holds clinical expertise and power; he or she controls and dictates the care provided to patients and their families. Thus, patients and their families are expected to listen and do what the nurse commands. 
A fundamental aspect of how people see the world is that they define themselves as members of a preferred group and create 'other' categories of groups where less preferred people belong (Hart \& Freeman, 2004). Under the medical model, nurses feel and believe that they are the preferred group with knowledge and power to control patients and their families. The potential for patient and family decision making and a holistic approach to patient care is therefore impossible (Martin \& Younger, 2000).

"For anti-oppressive practice to become possible the balance will have to shift in favor of the patient. It is the nurse who is able to bring about this shift, by insuring the patient's boundaries of knowledge and controls are extended" (Martin \& Younger, 2000, p. 60). FCC is a method that nurses can use as a way to move away from the medical model to a more anti-oppressive nursing practice. This is because FCC encourages the nurse to view the patient and family members as the expert, collaborate with them, and listen to their requests and concerns.

Parent's Perception of Family-Centered Care

FCC involves respecting and incorporating the parents and families in care;

therefore, it is important to take their perspectives into consideration when providing care to children. Behaviors that were demonstrated in studies conducted by Vincent et al., (1996), and Turner, Tomlinson and Harbaugh (1996) reveal that parents can identify good nursing care provided to their children. Understanding the perceptions of parents offers nurses insight in how to provide quality care to children that meet the needs of the family unit and is truly family-centered.

Vincent, Alexander and Patterson (1996), conducted a phenomenological study to describe the perceptions held by parents of nurse's caring behaviors in a Pediatric 
Intensive Care Unit (PICU). They used semi-structured interviews of ten pairs of mothers and fathers of ill children in four PICU's located in the Southeast United States. Caring behaviors were reported in four areas. The first identified caring behavior was the 'characteristic of the nurse,' which included cuddling the child, being nice, helpful, compassionate, expressing emotions, and possessing knowledge and confidence. The second identified behavior was 'meeting the child's needs', such as responding to alarms and changing diapers. Third, 'meeting the parents' needs' was described by providing information, encouraging parenting, and providing support. The fourth reported caring behavior was 'feeling touched by caring', such as, providing security, relief of stress, and the child being cared for.

In a study by Turner et.al, (1996), parents described the nurses that cared for their child as being notable because they demonstrated vigilance, efficiency, competence, protection, and nurturance. The confidence of parents in their child's nurse depended on whether the nurse talked to, held, or comforted their child. Such actions demonstrated special interest in their child and taking special consideration to alleviate the negative effects of the hospital stay. Also, parents identified it as important for the nurse to extend their empathetic attitude to parents and families.

Harbaugh, Tomlinson and Kirschbaum (2004) conducted a qualitative study to describe parents' perceptions of nurses' care giving behaviors in the Pediatric Intensive Care Unit. Two major behavioral groups emerged: Vigilance: Watching and Protecting, and Nurturance: Giving Affection and Caring.

Vigilance: Watching and Protecting included the nurse watching in a way that the parents could see that their child was cared for and protected against error or mishaps. It 
brought peace to parents when nurses made it possible for the parents to be present during procedures, whereas, when parents were not allowed to be present negative consequences occurred. When watching was combined with open communication and information sharing, parents felt their child was receiving the best protection.

Nurturance: Giving Affection and Caring lessened the negative impact parents felt as they lost their role as a parent during their child's hospitalization.

Parents perceived some nurses as genuinely appreciating the humanity, the uniqueness, and the vulnerability of their child when they observed nurses being emotionally warm to the child, when they touched the child affectionately, and when they were able to inform them of everyday indicators of the child's personality and comfort. (p. 171)

Communication of the child's condition also decreased parental anxiety.

Examples of nurses' behaviors that help to make the unfortunate situation of a child's hospitalization more manageable for parents include: (1) allowing access and maintaining proximity to the child; (2) reducing stress and uncertainty with ongoing, accurate information and reassurance; (3) conducting nursing care in a competent, coordinated manner; (4) appreciating the individuality of the child; and (5) keeping parents informed of their child's daily responses and progress (Harbaugh, Tomlinson \& Kirschbaum, 2004).

MacKean, Thurston and Scott (2005) identified what parents expect of FCC in the caring process through their grounded theory research study. Parents desired to have a more input when their roles were defined, and more help from health-care professionals in making decisions about services, ongoing management, and the ability to advocate for their child's best interest. Parents valued relational competency of health-care professionals above technical, treatment and diagnostic competencies. Relational 
competencies included: caring, communicating with parents, and interacting with children. Caring involved being compassionate, respectful and providing care that was personalized. In MacKean et al.'s study (2005), it was found that health-care professionals valued providing information over their role as a caring person, collaborator and helper. This study demonstrated that health care personnel value different aspects of FCC in comparison to parents. This reinforces the importance of nurses being aware and understanding of parents' perceptions of FCC when providing care to children.

Parents identified the following important factors of FCC in Regan, Curtin and Vorderer's (2006) study: communication, responsiveness, honesty and emotional support. Miles (2003) found that parents want to be kept informed on their child's progress because mistrust in caregivers can occur when there are delays in obtaining answers to questions or when annoyance is conveyed by professionals.

According to Kristensson-Hallstrom and Elander (1997) some parents actually feel like outsiders in settings where FCC is not regularly practiced. Also, such parents feel that in order to ensure their child receives the best quality of care they have to limit their preferred level of involvement (Kristensson-Hallstrom \& Elander, 1997). Therefore, when FCC is not implemented, parents feel like a stranger to their own children and as though they cannot participate or contribute to their own child's health and recovery.

Regan et al. (2006) found that parents need emotional support from nurses. In order for parents to care for their child, they feel that they themselves need to be cared for and require nurses to acknowledge that they are undergoing great stress from their child's hospitalization. According to Meleski (2002), nurses can care for parents in the following ways: (1) assist parents in coping with a child's illness, (2) encourage family members to 
express their needs, (3) identify existing support systems outside the family, (4) assist parents in accepting their child's condition, (5) assist parents in developing realistic expectations, and (6) help the family to adapt and reorganize in order to meet the child and family's needs.

The parent's caregiver role changes when their child is hospitalized (Ryan \& Steinmiller, 2004). As a result, they are unable to hold, protect and comfort their child during unfamiliar and painful procedures, which causes them to feel helpless (Ryan \& Steinmiller, 2004). Such helplessness can contribute to an increase in the family's level of frustration and anxiety (Scott, 1998). When nurses provide FCC they have the potential to alleviate such frustration and anxiety of parents by involving them in care and decision making.

Identifying and understanding parent's perspectives of FCC is important for nurses to be aware of in order to truly practice FCC. The above studies illustrated the actions and attitudes parents feel nurses should incorporate into their practice so that they can comfortably place their child in the hands of health care professionals. Unfortunately, FCC is not always practiced the way parents feel is best. The following section explains the current lack of FCC in pediatric nursing.

Despite the benefits of FCC, it is often lacking in many pediatric health care settings. Reasons for its lack in practice are discussed in the following section.

Understanding the Lack of Family-Centered Care in Pediatric Nursing: Challenges and Barriers

FCC has been established as vital to effective children's health care (Hemmelgarn, Glisson \& Dukes, 2001). The positive benefits of providing emotional support to patients 
and their families through the implementation of FCC have been confirmed.

Unfortunately, such practices do not exist in all medical settings, including pediatrics (Hemmelgarn, Glisson \& Dukes, 2001). There are barriers and challenges that hinder nurses from implementing FCC in their practice.

Not all pediatric nurses incorporate FCC in their practice. According to Bruce and Ritchie (1997), some researchers have found that "nurses do not view the needs of the family as their responsibility," and may lack the skills and knowledge needed to provide FCC (p. 214). As a result, parents often believe health care professionals lack respect for and recognition of their expertise as caregivers.

Frequently, health care professionals do not operate according to the philosophical framework of FCC (Bradshaw, Coleman \& Smith, 2003). Parents are involved in various degrees of care and treated differently according to what each individual nurse practices. Thus, families have the potential to receive care that is confusing and disjointed (Bradshaw, Coleman \& Smith, 2003). Such instances can be damaging to the child's therapeutic progress and prevent families from developing trusting relationships with health care professionals (Bradshaw, Coleman \& Smith, 2003) According to McGrath (2001), some nurses believe that providing FCC interferes with the care given to the patient, induces job stress, and is simply not part of their job. This is supported by Bruce and Ritchie's descriptive study (1997), as they found that "nurses saw collaboration with families as being the least necessary and least practiced element of family-centered care" (p. 220). Bruce and Ritchie (1997) also found that "nursing practice continues to reflect inconsistencies in the level of desired FCC of 
children and their families" and nurses did not have expectations of such or provide such care (p. 219).

Brown and Ritchie (1990) found that nurses feel that they need to be gatekeepers regarding the extent of care parents should provide, and how many decisions parents should make while their child is hospitalized. For many years, professionals thought it was in the best interest of families to make the decisions and be in total control of the nursing care provided to children (Shelton \& Stepanek, 1994). FCC challenges such practice because it emphasizes the importance of helping patients and families build on their own strengths and share in opportunities that enhance their involvement (Regan, Curtin \& Vorderer, 2006).

According to Galvin et al. (2000), health care providers are afraid that adhering to FCC principles will threaten their professional identities. Pediatric nurses are in a position of power and it can be intimidating for them to give up such power and control. Nurses often define themselves and find security in their job because of such authority, but FCC encourages nurses to share control with the child and their families. Nurses' values and philosophical biases can impact the implementation of FCC (Regan et al., 2006). Therefore, nurses who value such authority likely do not fully implement FCC into their practice.

Nurses are caring for increasing numbers of patients and families from various ethnic and cultural backgrounds that are unlike their own (Craft-Rosenberg, Kelley \& Schnoll, 2006). Also, nurses find it hard to understand families' needs and prioritize because nurses and the families do not always have the same values (Ryan \& Steinmiller, 2004). As a result, nurses can find it difficult to create trusting relationships and offer 
true collaboration to patients and their families when they have different ethnic backgrounds, values and beliefs than those of the patient and family. The principles of FCC encourage nurses to listen to the values and beliefs of the patient and their families and incorporate their cultural background into the care provided, and as a result a trusting relationship will be formed.

The medical model is still influential in our health care system today (MacKean, Thurston \& Scott, 2005). Included in the medical model are strong socially defined roles of health-care professionals and families; it is a professional expert model where the professional holds the knowledge and authority without considering input from the patient or family (MacKean, Thurston \& Scott, 2005). "Rather than developing collaborative relationships characterized by open communication, trust, and negotiation, there is a tendency for health professionals as experts to define family-centered care and the roles that parents are expected to play" (MacKean, Thurston \& Scott, p. 82). The medical model leaves little control and flexibility for the family's input because the nurses assume responsibility for the problem and orchestrate care (Bruce \& Ritchie, 1997). Therefore, the current medical model is a barrier to FCC because collaboration and teamwork with families are core principles of FCC.

Pediatric nurses simply lack the time to offer family-centered care at all times (Craft-Rosenberg, Kelley \& Schnoll, 2006). Nurses feel their efficiency is compromised if they are asked to include families in procedures, reallocate or increase time spent on tasks necessary to include, and instruct parents during treatments (Regan, Curtin \& Vorderer, 2006). Elements of FCC are less interested in in-the-moment efficiency, but it emphasizes taking time to address parent's anxiety (Regan, Curtin \& Vorderer, 2006). As 
a result, nurses may find it difficult to consistently implement FCC into their practice because nurses are pressed for time.

\section{Current Difficulty Implementing Family-Centered Care}

There is a lack in clarity of FCC in both the literature and practice (MacKean, Thurston \& Scott, 2005). There are various definitions and theories of what encompasses FCC. Upon conducting the literature review for this research study, there was a lack of concrete examples and ways to implement FCC into nursing practice.

Patterson and Hovey (2000) question whether the philosophy of FCC has actually taken hold in practice and policy, or if it is just rhetoric. This is because they found that families feel unheard and little seems to have changed in the pediatric care setting. Thus, FCC has not been implemented as intended among pediatric health care providers because if it was, families would have reported an increase in satisfaction with care provided to their children, not state they feel unheard.

Research studies show that the extent to which FCC is practiced among nurses is a function of their experience, education and orientation of the individual provider (Hemmelgarn, Glisson \& Dukes, 2001). Also, research has discovered that the differences in the practices and perceptions of FCC is a function of the health care professional's education, age, and individual characteristics (Hemmelgarn, Glisson \& Dukes, 2001). Therefore, such factors play a key role in the successful implementation of FCC in practice: when these factors are lacking, so is FCC.

According to Shields (2007), it is important that rigorous evidence supports health care practices, therefore, "health care practitioners must critically examine familycentered care and how it is delivered" (p. 869). Pediatric nurses need to attain particular 
skills, knowledge and experience in order to implement FCC into their practice adequately; such information does not appear to have been given consideration (Baker, 1995). There is a lack of research on how FCC is delivered, as well as, a lack of literature that provides knowledge and experience on FCC. This contributes to the current difficulty in implementing FCC into nursing practice.

Hutchfield (1999) conducted a concept analysis of FCC. She discovered during her field work that nurses were unsure of whether they were required to facilitate a relationship between themselves and the family and if they had time to help families with problems that were not related to the ill child. Also, co-operation, collaboration and negotiation of care were not always evident in the care provided to children and their families. Hutchfield's (1999) findings imply that there are difficulties implementing FCC; therefore, further research is required.

Outcome research revealed that FCC decreased child and parent anxiety, increased patient and family satisfaction, had a positive impact on the mental health of mothers with ill children, decreased recovery time from medical procedures, increased staff satisfaction and decreased healthcare costs (American Academy of Pediatrics, 2003). However, the interpretation and implementation of FCC differ among institutions and practitioners (Regan, Curtin \& Vorderer, 2006). In order for health care professionals to fully provide FCC, practitioners must alter institutional structures and policies to encourage and inform such types of practice. The following section will address the lack of institution support for FCC. 


\section{Lack of Institutional Support for Family-Centered Care}

According to Galvin et al. (2000), there is not strong support from an organizational perspective to implement FCC into nursing practice. In order for FCC to be put into practice, the institution in which health-care professionals work must support and encourage such practices. Earlier research also established that an institution's commitment to FCC influences the operationalization of such practices (Bruce \& Ritchie, 1997). Thus, nurses require guidelines, standards, and educational opportunities to practice FCC.

A qualitative study was conducted by MacKean et al. (2005) at the Alberta Children's Hospital to develop a conceptualization of FCC. It was found that health-care providers recognized that the system was not user-friendly; parents and health-care providers felt that there was a need for system change. These changes included help with advocacy and care coordination, information about services, coordination of service transition, coordination within and between services, increase resources and services that are truly driven by family needs.

According to Titone et. al, (2004), in order for an organization to efficiently provide FCC, the following ought to be acknowledged. The organization must focus on family strengths and not deficits. It should be a collaborative partnership model where parents are free to provide vital input and not a medical model in which professionals have total control. The organization should be a model of supporting patients and family's positive attributes rather than focusing on their negative aspects. It is essential that the organization moves away from a model of rigidity and patient dependence to a model of empowerment and flexibility. This entails a model of information sharing that 
does not "gate-keep" by the nurses, but encourages collaboration and communication with the patient and their families (Titone et al., 2004).

One way that institutions can support nurses with the implementation of FCC is by creating educational opportunities that inform and educate them on FCC. LeGrow and Rossen (2005) implemented an 8-hour education day on the Family-Systems Nursing Framework and provided nurses with various types of material on this topic. Following the education sessions, LeGrow and Rossen (2005) completed an evaluation project to elicit nurses' and families' experiences of the changes and differences when nurses follow a family systems nursing approach to guide practice. As a result of this education, nurses had an increased focus on nursing the family as a whole unit, had an increased ability to resolve problems effectively, changed their thinking in regards to caring for the family, had an increase in their work satisfaction, and found meaning in their practice. Family-nurse relationships were characterized as being more open, supportive, caring, mutual, trusting, helpful, respectful and positive. Nurses reported taking more time to listen to families' concerns. Families noted there were improvements in their emotional and psychological health, an increase in friendliness from nurses, an increase in frequency of conversations, consistency of nursing care and a better rapport with nurses. There was a reported decrease in family problems, family complaints and days spent in hospital. As shown, education opportunities on FCC increase the implementation of FCC in nursing practice. Also, such educational opportunities result in an increase in patient and family satisfaction, as well as an increase in nurse's satisfaction.

As previously noted, the institution in which nurses practice greatly influences the way that care is delivered. When an institution supports and encourages nurses to provide 
care that is family-centered, there are positive results. Now that FCC has been discussed and explained in detail, including the barriers and parent's perspectives, FCC in the context of pediatric oncology nursing will be discussed.

\section{Pediatric Oncology Nursing and FCC}

\section{Childhood Cancer Statistics}

Approximately 850 children in Canada, between the ages of birth - 14, are diagnosed with cancer each year (Canadian Cancer Society, 2008). The number of deaths from childhood cancer is one-sixth of the number of newly diagnosed cases (Canadian Cancer Society, 2008). Cancer incidence rate among children has been relatively constant since 1985, but the number of deaths continues to decline (Canadian Cancer Society, 2008). The improvement in childhood cancer survival is due to several factors: development of multi-modal therapies, centralization of care and support services, and better diagnostic procedures (Canadian Cancer Society, 2008). The current survival rate among childhood cancer is now $82 \%$, which increases the need for long term follow-up of survivors because of the side effects from harsh cancer treatment regimens (Canadian Cancer Society, 2008). Cancer among children creates a disproportionate impact on health, social and economic welfare systems as a result of losing young lives (Canadian Cancer Society, 2008). As noted above, childhood cancer is an important health issue among Canadians today.

"Tumors in children have short latency periods, often grow rapidly and are aggressive, invasive and frequently spread to other parts of the body" (Canadian Cancer Society, 2008, p. 60). The most common type of cancer in children is leukemia, followed by cancer of the central nervous system and lymphomas respectively (Canadian Cancer 
Society, 2008). In Canada, it is estimated that $80 \%$ of children diagnosed with cancer are enrolled in a clinical trial or are treated according to a standard treatment that was developed from a clinical trial (Canadian Cancer Society, 2008).

Stress from Childhood Cancer

"When a child is diagnosed with cancer, the cancer becomes a threat to the child's life and to the family's security" (Björk, Nordström, \& Hallström, 2006, p. 211). The cancer experience affects the individual as well as the family as a whole; this is especially true with childhood cancers (Kerr et. al, 2007). According to Woodgate and Degner (2003), childhood cancer has an intense impact on the lives of children and their families because it forces them to face many stressors and challenges.

A common result of a child's illness is an increase in stress experienced by parents (Trask et. al, 2003). Children with cancer and their families experience life-long consequences and emotional trauma (Canadian Cancer Society, 2008). Cancer treatment is carried out over long periods of time and is often painful and strenuous (Björk et al., 2006). Families work less because they often stay home with their sick child, which places great stress financially on the family unit (Canadian Cancer Society, 2008).

Children with cancer experience great distress as they undergo treatment and face the many challenges of living with cancer. Hedstrom et al. (2003) conducted a study to identify distressing events that children and adolescents with cancer undergo. They found that physical aspects of distress include; pain from diagnostic procedures and treatments, fatigue and nausea. Emotional aspects of distress included feelings of alienation, confinement, worry before medical procedures and worry about death. 
Treatments such as chemotherapy, radiation, surgery and bone marrow transplants are exhausting treatments that children with cancer undergo (Clarke \& Fletcher, 2003). Such intense therapy has increased the amount and intensity of side effects that children with cancer experience (Fochtman, 2006). Such side effects involve the physical, psychosocial and spiritual; all which have increased the complexity of care (Fochtman, 2006).

Björk, Wiebe and Hallström (2005) conducted a phenomenological study to elucidate the family's lived experience of having a child in their family diagnosed with cancer. They discovered that all that was safe, secure and familiar in the family's life was replaced by fear, uncertainty, loneliness and chaos. The families described feeling like their life world was falling apart and that they lost their foothold in life; they became vulnerable and aware of mortality (Björk et al., 2005). Family members felt as though they were "thrown into the unknown" and that life became intimidating because it was as though life came to a standstill. Parents expressed great longings to escape because it all felt unreal, but they could not escape, because this was their new real life.

Parents found it extremely challenging and heartrending when their child begged for help to escape unpleasant or painful situations (Björk et al., 2005). One mother's description of such situation is as follows:

She would scream at me, Mommy please get me out of here! I felt like I wanted to take my child away from there. Don't hurt my child, but at the same time you know that you have to let them [the staff] take those tests. They do it in the best, easiest way, but at the same time it is horrible to have to see your child go through what they have to. (p. 270)

Parents feel helpless as they watch their child suffer from cancer. "The very nature of cancer, with its uncertain prognosis and painful treatments, contributes to the 
vulnerability and powerlessness that parents feel” (Holm, Patterson \& Gurney, 2003, p. 310).

Gibson et al. (2005) found in their phenomenological study that children with cancer who undergo intense treatments experience fatigue. Fatigue was described as "absolute and complete exhaustion experienced after treatment" (Gibson et al., 2005, p. 654). Such fatigue left the children weak, inactive, unmotivated and unable to participate in normal activities because of aching and painful limbs (Gibson et al., 2005). Gibson et al. (2005) also found that children with cancer experienced mental fatigue, inability to sleep, social isolation, apprehension and confusion about life itself, nightmares about treatment, and stressful visions that disrupted any sense of rest and tranquility. Children with cancer still want to play and enjoy their childhood; as a result of intense treatment they no longer can.

The diagnosis and treatment of cancer among children is a traumatic experience for them and their families; as well the "families face many changes to their everyday life" (Mitchell, Clarke \& Sloper, 2006, p. 805). The entire family is affected by the many demands of the illness and treatment (Wilkins \& Woodgate, 2005). Families are forced to adjust to the reality of a devastating diagnosis and balance family functioning all at the same time (Wilkins \& Woodgate, 2005). Pediatric oncology nurses are in an optimal position to provide strength and comfort in a time such as this.

\section{Nursing Pediatric Oncology Patients}

There have been many advances in treatment outcomes and survival rates among children with cancer, which has influenced the objectives and goals of nursing care for such children (Cantrell, 2007). Currently, pediatric oncology nursing is focused on 
implementing clinical trial research protocols, providing optimal supportive care, providing psychosocial support during treatment, and educating families about the short term and long term side effects of chemotherapy (Cantrell, 2007). All of these activities are to enhance the quality of the patient's life during therapy and survivorship (Cantrell, 2007).

The advances in medical treatments for children with cancer means that "the nature of care provided in inpatient, outpatient and community settings continues to change, with clinical advances, and children with cancer requiring highly specialized care" (Tomlinson, 2004, p. 646). Such advancements make it more difficult and complex for nurses to care for patients and their families.

Holm, Patterson and Gurney (2003) conducted a qualitative study to address the gap in the nursing literature regarding the experience of caring for a child with cancer. They hoped to provide additional information and insight for medical professionals who promote parental involvement in their child's care. It was found that parents often advocate for their child by making critical decisions and were "an incredibly important member of their child's medical team" (p. 304). Parents advocated for their child because no one knew what their child was feeling or going through as well as they did (Holm et al., 2003). However, parents often found it difficult to advocate for their child because parents had limited knowledge of medical procedures and terms (Holm et al., 2003). Also, parents did not know when it was okay to press the medical team with questions or concerns because they were afraid of being disrespectful (Holmet al., 2003). Pediatric oncology nursing involves working with parents over long periods of time and involving them in the medical team as advocates. Therefore, it is important to create trusting 
relationships with the parents of children with cancer so that they can feel comfortable advocating for their child.

Parents truly know their child best. Thus, it is important for the medical staff to listen to parents when they are worried about their child. In the study by Holm et al. (2003), one third of parents had to be persistent in order for their child to receive an accurate diagnosis after they recognized that their child was unwell with unusual symptoms. Often times, parents had to continue to bring their child to the hospital and demand further testing in order for treatment to begin (Holm et al., 2003). If these parents had not been persistent, their children would not have been properly diagnosed and started on life saving treatment. Hence, parents have insight into their child's health and well-being that medical staff must be open to and consider when providing care to children with cancer.

Holm et al. (2003) found that what sets parents of children with cancer apart from other parents "is that they face a life-and-death situation, which added to their vigilance, active support, and intercession on their child's behalf' (p. 310). When parents are able to advocate for their children, they feel that they are protecting their child in the best way possible, and are "able to restore some small sense of control" (Holm et al., 2003, p. 310). Parents are the expert on their child's care, therefore, it is important to listen and include parents in the care by allowing them to actively advocate for their child. Medical staff should collaborate with parents in the decision making process in order to make informed decisions that truly includes the child's best interest. One mother stated it as follows:

I think that when they [parents] are first brought in and they're told all these thousands of things, they need to be told what their role is. People don't know what their role is.... So right away, the medical team needs to identify them as you're an important part of this team, a nonmedical person, but there's a lot of 
nonmedical people on your team - and tell them how important it is to be that advocate, to learn what they can, to pay attention, to document. (Holm, Patterson \& Gurney, 2003, p. 311-312)

Parents express that they experience great chaos when their child is diagnosed with cancer and undergoing treatment (Björk et al., 2005). Therefore, it is difficult for them to understand information that is given to them at such times. In Björk et al. (2005) phenomenological study, one mother noted that "the doctor kept saying over and over again that $75 \%$ of those who got leukemia survived, but it took several days before she understood that it was possible to survive following childhood cancer" (p. 271). Also, when the nursing staff was in a hurry, information was unclear and inconsistent; this caused parents to experience an increase in chaos (Björk et al., 2005). Increasing a parent's chaos is absolutely not what pediatric oncology nurses intend to do. Unfortunately, parents experience chaos when their child has cancer because today's health care system is focused on treatment and technology rather than caring for patients and their family's holistic needs. FCC is a means to reduce chaos experienced by parents because it seeks to create therapeutic relationships and provide clear and understandable information.

The Association of Pediatric Oncology Nurses has placed an increased emphasis on nurses to be knowledgeable regarding the health-related quality of life (HRQOL) for children with cancer during treatment (Cantrell, 2007). An adequate HRQOL can be accomplished if oncology nursing practice is based on a caring and therapeutic nursepatient-family relationships (Cantrell, 2007). FCC promotes and fosters the development of a caring nurse-patient and nurse-family relationship. Therefore, it is important for pediatric oncology nurses to incorporate FCC into their practice. 


\section{Family-Centered Care among Pediatric Oncology Nurses}

FCC is important to pediatric oncology nursing because it encompasses caring for children with parents who are highly involved. Often times, FCC is not easily implemented into pediatric oncology nursing practice because nurses are under pressure to perform specialized tasks, such as the administration of chemotherapy drugs from protocols and clinical trials. "If the artistic nature and caring presence are stripped from the practice of pediatric oncology, patients and families will surely suffer, and positive treatment outcomes will decline" (Cantrell, 2007, p. 137). Therefore, it is important that pediatric oncology nurses do not become strictly task orientated and lose sight of the holistic and human aspects of nursing practice. FCC is a vehicle that pediatric oncology nurses can use to ensure that their care encompasses the holistic aspects of patients and their families.

Schwarts (1995), a 40-year-old health care lawyer who fought cancer, publicly expressed gratitude for "moments of exquisite compassion" and an "extraordinary array of human and humane responses" from the medical team during his cancer treatment (p.1). To Schwartz (1995), "quiet acts of humanity have felt more healing than the highdose radiation and chemotherapy that hold the hope for cure" (p.2). As a result of his experiences, Swartz founded a center that is dedicated to strengthening the relationship between the patient and caregiver. Thus, it is important that oncology nurses do not become primarily focused on the treatment aspects of cancer care, but also on the simple acts of humanity that bring about holistic well-being. FCC is a philosophy nurses can use to guide their practice because it will enhance the relationships that they create with their patients and their families. 
"Pediatric oncology nursing is based on family-centered care" (Ogle, 2006, p. 29). The children and their families become the heart of pediatric oncology nursing because such children live within the context of their families (Ogle, 2006). When nurses care for children with cancer, they provide care that is family-centered, because the family is going to be a constant support that enables the child to endure the long journey that cancer treatment brings.

A qualitative study conducted by Clarke and Fletcher (2003) revealed that parents of children with cancer felt that they know their child better than the health care providers; but they did not know medical oncology, its practice, or its science. Thus, parents and health care providers are required to work together as a collaborative team in order to care for children with cancer. A relationship with open and honest communication is needed to ensure that child's needs are being met.

In order to provide care to children with cancer that is family-centered, nurses must recognize that the one-size-fits-all approach does not work (Ogle, 2006). Care must be tailored to meet the unique needs of each patient and family, on and individual basis. "Nurses and other health care professionals must work collaboratively with families to identify and implement the best approach to treatment" (Ogle, 2006, p. 31).

Parents of children with cancer are greatly involved and responsible for their child during treatment periods. Mothers and fathers are involved in the provisions of care such as; chemotherapy administration, central line hygiene, maintaining oral care, and monitoring the various side effects of treatments (Clarke \& Fletcher, 2003). Also, when children are in hospital, health care professionals rely on help from parents for child management, monitoring of symptoms, child minding, and taking tests and treatments 
(Clarke \& Fletcher, 2003). According to Holm et al. (2003), parents are included in the care for their child as a coach and co-therapists to reduce pain, distress, and anxiety during cancer-related medical procedures such as lumbar punctures, venipunctures, intramuscular and intravenous injections, bone marrow aspirates, and accessing ports. Parents play a large role in the care provided to their children, thus nurses and other health care providers must be aware and knowledgeable on the principles of FCC when caring for children with cancer.

"Good communication is essential for good outcomes when a child is diagnosed with cancer" (Sobo, 2004, p. 150). Pediatric cancer involves toxic pharmaceuticals and medication regimens that change frequently; this demands good communication between health care professionals, patients and families in order for the child to receive safe care (Sobo, 2004). Miscommunication can lead to medication errors and negatively impact adherence to treatment protocols. The philosophy of FCC includes open and honest communication; thus, it is important to incorporate FCC in pediatric oncology nursing practice as a way to ensure parents are informed, miscommunication does not happen, and errors are avoided.

Childhood cancer is a life-threatening disease that many children must endure. According to Ward-Smith et al. (2005) "few health care professionals have the training or the time to fully address the social, spiritual, and psychological issues associated with life-threatening illnesses" (p. 321). As a result, health care professionals often only talk about cure rates and treatment options rather than holding discussions about what the family values, creating therapeutic relationships, and providing care that is sensitive to the individual needs of each patient and family (Ward-Smith, et. al, 2005). It is 
unfortunate that during such a sensitive and delicate time in a child and families lives, when they would need the most support and spiritual/psychological care, health care professionals are unable to do so. FCC is a philosophy that encourages health care professionals to listen to patients and their families talk about their values, thus enabling them to provide care that is sensitive to the social, spiritual and psychological needs of the patient and their family.

As demonstrated and discussed above, FCC is essential for the care of children with cancer. In concluding this extensive literature review, valuable information from a parent's perspective on caring for children with cancer will be presented to help the reader understand the importance of FCC among pediatric oncology nurses.

Valerie McDonald (1999/2000) wrote an article from a parent's perspective on how to provide compassionate and effective care to children with cancer. Two of McDonald's three daughters were diagnosed with cancer; one that has since passed from leukemia. During her experiences of having children with cancer she has encountered the "greatest frustration in areas of communication, coordination of care, consistency of care and trying to achieve care that addresses the needs of the whole person" (McDonald, 1999/2000, p. 20). McDonald states that effective communication is critical to good patient care, such as providing adequate information and taking time to answer questions. At times in her children's care, lack in coordination of care was simply unforgivable because of the pain her children had to endure as a result. Her and her family found it extremely frustrating to have a different nurse for each clinic visit because her daughters found it frightening to form new relationships on a daily basis. Most importantly, the most obvious way nurses showed care for the whole person was when her daughters were 
treated with respect and consideration; when staff took time out of their busy workday to speak to her and her family and learn how to address their needs.

From McDonald's article (1999/2000), ways that nurses can potentially provide FCC to children include: incorporate honest and open communication, ensure that the coordination between services runs smoothly, have consistent teams of health-care professional's care for children and care for the whole person by treating them with dignity and respect. McDonald stated that one of the worst things about having children with cancer was being forced to give up control during a time in her life when she most needed it (1999/2000). When health-care professionals provided FCC, it gave her back some control in her life and made her experiences of caring for two daughters with cancer more bearable. Pediatric oncology nurses are truly privileged and in an extraordinary position to help children with cancer and their families endure and carry on through a dreadful and vulnerable situation.

\section{Summary of the Literature}

FCC is at the core of pediatric nursing. Understanding and acknowledging that parents are the experts in their child's health is central to providing FCC. Unfortunately, not all pediatric nurses incorporate FCC into their practice because there are barriers and challenges that prevent them from doing so. Such barriers and challenges include: the prevalence of the traditional medical model, nurse's lack of understanding regarding FCC, time pressures on nurses, medical technology, staff shortages, an increase in the cultural diversity of patients, nurses' values and beliefs, absence of formalized FCC policies, and an increase in level of job related stress. 
Children with cancer and their families experience tremendous stress as a result of the various treatment regimens and plethora of tests they must undergo. Caring for such families can be complicated and requires specific skills and expertise. FCC among pediatric oncology nurses is germane because the families are extremely involved in the care that their child receives. It is hoped that these barriers and challenges can be identified in this study as a means to better incorporate FCC into the practice of pediatric oncology nurses.

The following chart highlights seven major studies that this literature review was built upon. These studies were central to the literature review. They were placed in chart form to summarize and highlight the key points of each study. The studies included in this chart provide an overview of research conducted regarding FCC up to date. 
Chart 2.3

Summary of the Literature

\begin{tabular}{|c|c|c|c|}
\hline Author(s) and Year & Purpose & Method & Major Findings \\
\hline Bruce \& Ritchie (1997) & $\begin{array}{l}\text { To examine pediatric nurses' } \\
\text { perceptions and practices of } \\
\text { FCC. }\end{array}$ & $\begin{array}{l}\text { Exploratory and descriptive: } \\
\text { using the Family-Centered } \\
\text { Care Questionnaire }\end{array}$ & $\begin{array}{l}\text { The nurses perceived that they performed all elements significantly less in their current practice } \\
\text { than they believed necessary. Perceptions about necessary components varied with age and clinical } \\
\text { position. Nurses indicated knowledge of FCC, but reported that they did not incorporate this } \\
\text { knowledge into practice. There was a lack of organizational guidance and direction, of recognition } \\
\text { for professional contribution, of confidence in and support for the professional autonomy of } \\
\text { nurses, and of the skills to integrate knowledge to perform FCC. }\end{array}$ \\
\hline $\begin{array}{l}\text { American Academy of } \\
\text { Pediatrics (2003) }\end{array}$ & $\begin{array}{l}\text { To provide a definition of } \\
\text { FCC. }\end{array}$ & $\begin{array}{l}\text { No method used. This paper } \\
\text { provides an in depth } \\
\text { explanation and definition of } \\
\text { FCC. }\end{array}$ & $\begin{array}{ll}\text { Nine core principles of FCC exist. They are: } \\
\text { 1. } \\
\text { 2. } \\
\text { 3. Hospect each child and his or her family } \\
\text { Support and facilitate choice, for the child and family about approaches to care and } \\
\text { 4. } \\
\text { 5. } \\
\text { 6ecognize and build on strengths of child and family } \\
\text { E. Share hoxesibility in policies, procedures and provider practices } \\
\text { 7. } \\
\text { 8. Provirming } \\
\text { 9. } \\
\end{array}$ \\
\hline $\begin{array}{l}\text { Holm, Patterson \& Gurney } \\
\text { (2003) }\end{array}$ & $\begin{array}{l}\text { Explore parent's descriptions } \\
\text { of their participation in } \\
\text { medical care for their children } \\
\text { with cancer. }\end{array}$ & $\begin{array}{l}\text { Seven focus groups were } \\
\text { conducted with } 45 \text { parents of } \\
26 \text { children who had } \\
\text { completed cancer treatment. }\end{array}$ & $\begin{array}{l}\text { Parents emphasized their role as advocates during the diagnosis and treatment phases by informing } \\
\text { themselves about their children's medical conditions, making medical care decisions, limiting the } \\
\text { actions of medical professionals and affirming and supporting medical professionals. The results } \\
\text { emphasize the need to employ a family-centered approach in cancer care medical settings by } \\
\text { fostering and supporting the active inclusion of parents in their child's treatment and disease } \\
\text { management. }\end{array}$ \\
\hline $\begin{array}{l}\text { Petersen, Cohen \& Parsons } \\
\text { (2004) }\end{array}$ & $\begin{array}{l}\text { To determine nurses' } \\
\text { perceptions and practices of } \\
\text { identified elements of family- } \\
\text { centered care. }\end{array}$ & $\begin{array}{l}\text { Survey, Family-Centered Care } \\
\text { Questionnaire (FCCQ). } \\
\text { Descriptive and Inferential } \\
\text { statistical analyses were } \\
\text { performed on data. }\end{array}$ & $\begin{array}{l}\text { Nurses understand and believe in the value of FCC but do not seem to execute the concept when } \\
\text { caring for children and families. Organization barriers to health care system design and lack of } \\
\text { guidance and direction were the biggest barrier. It is not sufficient to merely endorse the } \\
\text { philosophy of FCC, resources must be dedicated. Nurses with less experience identified elements } \\
\text { of FCC more important and reported using them more often than more experienced nurses. This } \\
\text { study demonstrates a need for more education for nurses on FCC. }\end{array}$ \\
\hline $\begin{array}{l}\text { MacKean, Thurston \& Scott } \\
\text { (2005) }\end{array}$ & $\begin{array}{l}\text { To develop a } \\
\text { conceptualization of FCC } \\
\text { grounded in the experiences of } \\
\text { families and direct health-care } \\
\text { providers }\end{array}$ & $\begin{array}{l}\text { Qualitative methods following } \\
\text { the grounded theory tradition. } \\
\text { Data collection was through } \\
\text { focus groups and semi- } \\
\text { structured interviews. }\end{array}$ & $\begin{array}{l}\text { Findings strongly support the importance of the relational component of FCC, specifically the } \\
\text { development of a true collaborative relationship between families and health-care providers. } \\
\text { Parents want to work collaboratively with health-care providers and work on implementing a } \\
\text { dynamic care plan that will work best for the child and family. }\end{array}$ \\
\hline Shields, Pratt \& Hunter (2006) & $\begin{array}{l}\text { To systematically review } \\
\text { qualitative studies on the use } \\
\text { of FCC in children's hospitals. }\end{array}$ & $\begin{array}{l}\text { Systematic Review; papers } \\
\text { found in a wide range of } \\
\text { databases, hand searching and } \\
\text { contacting authors when } \\
\text { necessary. }\end{array}$ & $\begin{array}{l}\text { Negotiation between staff and families, and perceptions held by both parents and staff roles } \\
\text { influenced delivery of FCC. A sub-theme of cost of FCC to families and staff was discovered and } \\
\text { this included both financial and emotional costs. }\end{array}$ \\
\hline $\begin{array}{l}\text { Johnson, Abraham, Conway, } \\
\text { Simmons, Edgman-Levitan, }\end{array}$ & $\begin{array}{l}\text { To make recommendations for } \\
\text { partnering with patients and }\end{array}$ & $\begin{array}{l}\text { No methods used. This paper } \\
\text { was written for health care }\end{array}$ & $\begin{array}{l}\text { Four concepts of FCC exist. They are: } \\
\text { Dignity and Respect - Health care practitioners listen to and honor patient and family perspectives }\end{array}$ \\
\hline
\end{tabular}




\begin{tabular}{|l|l|l|l|}
\hline $\begin{array}{l}\text { Sodomka, Schlucter, \& Ford } \\
(2008)\end{array}$ & $\begin{array}{l}\text { families to design a patient- } \\
\text { and family-centered health } \\
\text { care system. }\end{array}$ & $\begin{array}{l}\text { professionals by the Institute } \\
\text { of Family-Centered Care to } \\
\text { education staff and leaders. }\end{array}$ & $\begin{array}{l}\text { and choices. Patient and family knowledge, values, beliefs and cultural backgrounds are } \\
\text { incorporated into planning and delivery of care. } \\
\text { Information Sharing - Health care practitioners communicate and share complete and unbiased } \\
\text { information with patients and families in ways that are affirming and useful. Information is timely, } \\
\text { complete and accurate. } \\
\text { Participation - Patients and families are encouraged and supported in participating in care and } \\
\text { decision-making at any level they choose } \\
\text { Collaboration - Patients, families, health care practitioners and hospital leaders must collaborate in } \\
\text { policy and program development, implementation, and evaluation; in health care facility design; } \\
\text { and in professional education, as well as in the delivery of care. }\end{array}$ \\
\hline
\end{tabular}




\section{CHAPTER THREE: RESEARCH DESIGN}

Research methodology represents a broad understanding of social-organizational context, ethical principles, and philosophical assumptions (Neuman, 2006). In contrast, the research method is a set of specific techniques that measures, observes, gathers and defines data; as well provides direction to reporting the results (Neuman, 2006). In this chapter the methodology and method used for this research study are addressed. Philosophical stance, data analysis and method, scientific rigor, and ethical considerations are also discussed.

\section{Methodology}

\section{Philosophical Stance}

Various paradigms are used to guide qualitative research studies. A paradigm is viewed as a set of basic beliefs that involves a set of ultimates or first principles (HesseBiber \& Leavy, 2004). It is a world view that defines the nature of the "world" and the researcher's place in it (Hesse-Biber \& Leavy, 2004). Paradigms are associated with the nature of reality (the ontological stance) and the relationship between the researcher and that being studied (the epistemological stance) (Cresswell, 1998).

Ontological Stance. Ontological stance identifies the nature of reality for the researcher (Creswell, 1998). In this study, my own paradigm or world view arises from constructivism. Constructivists believe that "realities are apprehendable in the form of multiple, intangible mental constructions, socially and experientially based, local and specific in nature" (Guba \& Lincoln, 2004, p. 26). Constructs are shared among many individuals within societies and across cultures, and their content and form are dependent the individual persons or groups who hold the constructions (Guba \& Lincoln, 2004). 
Constructs can be altered because they are not "true" in any absolute sense, but more or less informed or sophisticated (Guba \& Lincoln, 2004). Constructivist orientation assumes that the interactions and beliefs of people create reality; thus, reality is a product of social processes (Neuman, 2006).

Epistemological Stance: Epistemological assumptions address the relationship between the researcher and that being researched (Creswell, 1998). The constructivist paradigm assumes that the investigators and the participants are interactively linked, so that the findings are created as the investigation is carried out (Guba \& Lincoln, 2004). The voices and interpretation by the participants is key to understanding the phenomenon (Polit \& Hungler, 1997). Since the investigator and the participants are dependent on each other, knowledge is maximized when the distance between the inquirer and the investigator is minimized (Polit \& Hungler, 1997). The researcher emphasizes and acknowledges the dynamic, holistic and individual aspects of the participants.

Constructivists seek to understand the constructs that people hold and aim towards consensus, but are open to new interpretations as information improves and becomes more sophisticated (Guba \& Lincoln, 2004). The goal is for people to be more informed and formulate more sophisticated constructs, and to be aware of the meaning of competing constructions (Guba \& Lincoln, 2004).

This study used person-centered interviewing as a means to understand the constructs that pediatric oncology nurses at the ACH hold regarding the subject of FCC. The skills and techniques these nurses used to provide FCC were revealed and barriers and challenges also became evident through the data analysis. Person-centered 
interviewing was used as a means to create an interactive relationship with the participants so that meaningful findings could be created from the research process.

\section{Theoretical Underpinnings}

Arising from constructivism, the theoretical underpinning of this research study was one of naturalistic inquiry. "Naturalistic methods of inquiry attempt to deal with the issue of human complexity by exploring it directly" (Polit \& Hungler, 1999, p. 13). Researchers in the naturalistic tradition recognize the complexity of humans, the idea that truth is composed of multiple realities, and that humans have the ability to shape their own experiences (Polit \& Hungler, 1999). "Naturalistic studies result in rich, in-depth information that has the potential to elucidate the multiple dimensions of a complicated phenomenon" (Polit \& Hungler, 1999, p. 14). In this study, the experiences of pediatric oncology nurses in relation to FCC were explored directly through person-centered interviews as each nurse was a unique individual. It was recognized that each nurse had various realities that were shaped by his or her own experience; such as education, culture, values and beliefs. Collectively, however, the nurses provided insight into and understanding of FCC. Therefore, this study resulted in rich data that revealed the multiple dimensions of FCC among pediatric oncology nurses.

\section{Researcher's Personal Perspective}

I believe that as an implicated researcher it is essential that I share information about myself and my perspective and opinions about FCC. I am a 26 year old, married, of middle class living in Calgary, Alberta Canada. I attained my Bachelor's degree in Nursing from the University of Lethbridge in 2006. I am a staff nurse on unit 2 at the Alberta Children's Hospital. I am currently pursuing a Master's degree in Health 
Sciences from the University of Lethbridge. I have worked as a pediatric Registered Nurse for over three and a half years, 1.4 years as a research nurse within the hematology/oncology/transplant program at the $\mathrm{ACH}$, and have gained insight into what it is like to care for the pediatric oncology population.

It was through my pediatric nursing experience that I developed a keen interest in FCC. I came to realize the importance of incorporating FCC in my nursing practice as a way to provide quality care that meets the diverse needs of patients and their families. I have a great passion to provide care that is holistic and meets the emotional, spiritual, and psychological needs of patients and their families; not merely the physical. I found that FCC is a philosophy that aids me in providing such care.

I believe that families are greatly impacted by the illness of their children. I also believe that families know and understand their children best; they are the experts. Thus, it is important to listen and incorporate families in the care provided to their children. I understand that at times, nurses at the $\mathrm{ACH}$ are busy and experience great pressure and stress; as a result, they are unable to provide FCC. This understanding has given me a desire to explore how nurses provide care that is family-centered, the barriers and challenges that prevent them from doing so, and the extent to which the ACH encourages and supports nurses in this application of FCC.

\section{Method}

This thesis was an exploratory descriptive qualitative research study. The method used was person-centered interviewing (Levy \& Hollan, 1998). In this study, pediatric oncology nurses from the Hematology, Oncology and Transplant (HOT) program at the $\mathrm{ACH}$ were interviewed regarding their perspectives of FCC and the skills and approaches 
they used to provide such care. Finally, the barriers and challenges associated with the provision of FCC were addressed.

\section{Person-centered Interviewing}

Person-centered interviewing is a qualitative method of gathering and collecting data. Person centered interviews are not just samples of discourse, but are rather a way to "attenuate and disrupt ordinary and conventional patterns of social discourse" (Levy \& Hollan, 1998, p. 334). This method is used to understand experiences that go beyond role-determined surface scripts and to reveal the underlying elements of the organization of persons, socio-cultural environments and their interactions (Levy \& Hollan, 1998). Such interviewing is not made up of reliable techniques such as those used by scientific technicians to assure that what they find is valid and reliable (Levy \& Hollan, 1998). Rather, it is like a performing art; a musical score where you need to make use of social skills, psychological skills, social knowing and interpersonal interaction (Levy \& Hollan, 1998).

During person-centered interviews the interviewee is a respondent and an informant. The researcher engages the interviewee as an informant; one who is knowledgeable and can tell the researcher about the culture and behavior in their environment (Levy \& Hollan, 1998). The researcher also engages the interviewee as a respondent because they are the object of systematic study and observation (Levy \& Hollan, 1998). As the respondent, the researcher explores the interviewee's experiences, feelings, hopes and desires (Levy \& Hollan, 1998).

"Person-centered interviews move back and forth between the informant and the respondent modes" (Levy \& Hollan, 1998, p. 336). This was helpful for this study 
because during the interview the participants were informants who informed me about FCC, but were also respondents who shared their experiences and ideas about FCC within their own practice. This helped to provide an in-depth understanding of FCC that addressed both philosophical and theoretical, as well as, practice and "real" aspects of FCC. Methods such as phenomenology and narrative approaches do not necessarily provide the means for such information and data to be generated as effectively.

\section{Sample and Setting}

The population of interest was registered nurses and nurse practitioners who worked within the pediatric oncology program at the $\mathrm{ACH}$; both the inpatient unit and outpatient clinic. Twenty nurses were interviewed, and data saturation was attained as reoccurring categories and themes were distinguished after interview number 14 . The interview process continued after data saturation was attained because the interview appointments were already arranged. However, the six additional interviews validated the findings from the previous 14 interviews and further enriched the findings. Data for this study were collected directly from these registered nurses during the 2009 academic year.

There are no simple formulas for qualitative research that guide researchers in the size of their sample; generally they should use the largest sample possible (Polit \& Hungler, 1999). The larger the sample, the more representative of the population the data is likely to be (Polit \& Hungler, 1999). A sample size of 20 nurses was estimated for this study because typically, and with articulate participants, theoretical saturation can be attained (Morse, 2000).

This sample was a purposeful convenience sample, meaning the participants were recruited based on the most conveniently available people or subjects (Polit \& Hungler, 
1999). In addition, the sampling was purposeful, i.e., oncology pediatric nurses providing FCC. The limitations associated with convenience sampling is that the participants may be atypical of the population they represent. For example, the participants of this study may only have been nurses who support and practice FCC, therefore, not accounting for nurses who do not practice FCC and feel it is unnecessary.

\section{Access and Recruitment}

A poster (see Appendix A) with pamphlets (see Appendix B) was placed in the break room, report room and staff room on the in-patient oncology unit and the outpatient oncology clinic. The pamphlet outlined a brief description of the study and the inclusion criteria; as well, my name and contact information were on the pamphlet. Nurses who were interested in participating contacted me directly. The first 20 nurses who responded were added to the list of interviewees.

\section{Inclusion Criteria}

Prospective participants were registered nurses or nurse practitioners who worked on either the in-patient oncology unit or in the out-patient oncology clinic. Each nurse was registered with CARNA, was male or female, had any amount of nursing experience, was able to read, speak and understand English, and had the desire to talk about FCC and their related experiences. The participants were willing to voluntarily sign the informed consent form.

\section{Data Collection}

The pediatric oncology nurses participated in semi-structured, person-centered interviews. The interviews were conducted in a mutually agreed upon setting that was convenient and comfortable for the participant; all of the interviews occurred on the unit 
in an available report room. Each interview lasted approximately one hour. Informed consent (see Appendix C) and demographic data (see Appendix D) were obtained. Participants were provided with an opportunity to ask questions before beginning the interview. The participants were then asked a set of open-ended questions and were encouraged to share their experiences and extent to which they practiced FCC. An interview outline (semi-structured guide) was used to provide the interview with some structure (see Appendix E). The interview guide was created from the core concepts of FCC arising from the Institute of Family-Centered Care (2008) and did not gain content validity. I was attentive in allowing the interview to move in directions not expected. The interviews were digitally recorded and then transcribed verbatim.

After the interview I provided each participant with a gift certificate of $\$ 5.00$ to the local café in the hospital to demonstrate appreciation of their participation in this study. An opportunity was provided for the participants to debrief their experiences. If mentioned by the participant, I provided them with information about the Employee Assistants Program (EAP) within the Calgary Health Region. None of the participants asked for this service or demonstrated any sign of distress. The participants were thanked for voluntarily participating in the study at the commencement of the interview.

\section{Data Analysis}

"Qualitative analysis is a very labor-intensive activity that requires insight, ingenuity, creativity, conceptual sensitivity, and sheer hard work" (Polit \& Hungler, 1999, p. 573). The purpose of qualitative data analysis is to create order for a large amount of information as a way for the data to be synthesized, interpreted and communicated (Polit \& Hungler, 1999). Data analysis of qualitative data occurs simultaneously with data 
collection; the researcher searches for important patterns and themes right from the beginning of the data collection process (Polit \& Hungler, 1999). I accomplished this by journaling after each interview; ideas and possible themes were recorded in a diary. Qualitative data analysis is constructivist in nature because it involves putting together various segments of transcripts into a meaningful conceptual pattern (Polit \& Hungler, 1999). Meaningful patterns, concepts and themes were developed for this study through the process of thematic analysis.

Thematic analysis was conducted to analyze the data for this study (Polit \& Hungler, 1999). The transcripts were read carefully with an eye to identify any underlying patterns and clusters of patterns (Polit \& Hungler, 1999). Aggregating these patterns into concepts then took place. Related concepts were grouped together to develop a category scheme or theme (Polit \& Hungler, 1999). The data were then re-read for content and coded according to the themes identified. Occasionally, when reading the data for the third or fourth time it was discovered that the initial category system was inadequate or incomplete; new categories and/or themes were then created and previous themes were changed or altered (Polit \& Hungler, 1999). This process was facilitated by my supervisor and committee members.

Files were created on a computer and named according to each theme. The corresponding data were copied and pasted in each file as a way to organize the data so that all of the content of a particular theme was retrieved easily.

In qualitative analysis, "interpretation and analysis of the data occur virtually simultaneously" (Polit \& Hungler, 1999, p. 585). That is, the data were interpreted as they were categorized and thematically analyzed; the themes were integrated into a 
unified whole (Polit \& Hungler, 1999). The themes were used to describe and better understand the nature of FCC among pediatric oncology nurses, the challenges and barriers they experienced, how the institution supported or failed to support nurses in their endeavors, and the practical skills nurses used to incorporate FCC into their practice. It was anticipated that there would be contextual differences between the nurses who worked on the inpatient unit compared to the nurses who worked in the outpatient clinic. Such differences were anticipated because patients in the inpatient unit are more acutely ill, newly diagnosed or relapsed, which can be more stressful for nurses. The patients in the outpatient clinic are usually healthier and not as acutely ill, which can be less stressful for nurses. These potential differences were accounted for by separating out the data from nurses who worked in the outpatient clinic from data of nurses who worked in the inpatient unit. Transcripts for each care area were looked at separately to see if there were similarities or differences in the themes and concepts that were extracted from the data. It was noted during the data analysis that there were no differences between the groups; the practices of FCC were similar. These findings are noted further in Chapter 5, Discussion of the Findings.

Ethical Approval Process

Ethical approval to conduct this study was obtained from the Human Subject Research Committee at the University of Lethbridge and the Research Ethics Board (REB) at the University of Calgary, where the ethics for the ACH is located. An application form for ethical review of human subject research was completed and submitted to the Human Subject Research Committee and the REB. The Patient Care 
Manager (PCM) for the Oncology/Hematology Department at the ACH indicated full approval, in principle, of this study.

"Free and informed consent lies at the heart of ethical research involving human subjects" (Tri Council, 2005, p. 2.1). Therefore, a consent form was signed the beginning of each interview that addressed various facets of the ethical review process along with consent for recording and transcribing the interviews (see Appendix C). Participants' identities were kept anonymous because the data and audio records were coded with a pseudonym so that no personal information or names were present. The data and personal information, including a list of participants name and pseudonyms, were locked in a filing cabinet; only the project researcher had access to the information. After 25 years, the transcripts will be destroyed completely by shedding the paper, and the audio records will be deleted; which is a mandatory requirement of the REB at the University of Calgary. All paper-based data will be appropriately disposed of in a confidential waste bin.

The purpose of the study was explained in detail to each participant. He/she had an opportunity to ask questions and voice any concerns. The participants were informed that they were free to withdraw from the study at anytime, without reason, or negative consequences. In the following section, ethical considerations for this study are discussed further.

\section{Ethical Issues}

Ethical issues are the dilemmas, concerns, and conflicts that arise over the proper way to conduct research (Neuman, 2006). According to Neuman (2006), ethical conduct 
ultimately depends on the researcher; therefore, the following section includes how I ensured that actions were taken during the conduct of this study to ensure it was ethical.

"Free and informed consent must be voluntarily given, without manipulation, undue influence or coercion" (Tri-council, 2005, p. 2.4). I explained the purpose of the study and assured the participants that they had the right to withdraw from the study at anytime. Informed consent was attained by having the participants sign a consent form that laid out the terms and conditions of the study. I explained the details of the consent form thoroughly before their signature was obtained (See Appendix C). In signing the consent form, the participants verified that they had adequate information regarding the research, were capable of comprehending the information, had the power of free choice, and were voluntarily participating (Polit \& Hungler, 1997). "Throughout the process of free and informed consent, the researcher must ensure that prospective subjects are given adequate opportunities to discuss and contemplate their participation" (Tri-council, 2005, p. 2.5). Therefore, consent was an ongoing process throughout the length of this study. This study followed the principles of non-maleficence and beneficence. Nonmaleficence is the "duty to avoid, prevent or minimize harms to others" (Tri-council, 2005, p. i.6). This was accomplished by maintaining confidentiality of the participants. Beneficence is a "duty to benefit others and, in research ethics, a duty to maximize net benefit" (Tri-council, 2005, p. i.6). The anticipated outcome of this study is a better understanding of FCC from the perspective of pediatric oncology nurses.

The principles of beneficence and justice were be upheld by providing confidentiality and anonymity for research participants (Streubert \& Carpenter, 1995). The identity of the participants was kept anonymous; only I had access to participant's 
identification. Data was locked in a filing cabinet to which only I had access. The electronic files were locked on a computer with a password that only I knew. The participants' names were not present in the thesis or publications because pseudonyms were used.

The topic of FCC among pediatric oncology patients could have included sensitive issues for the participants. These sensitive issues could have placed participants in a vulnerable situation as I asked probing questions to gain an in depth understanding of their experiences and the phenomenon of FCC (Streubert \& Carpenter, 1995). Contact information for the Employee Assistance Program within the Alberta Health Services was provided so that I could refer the participant if needed; however, it was not required for any of the participants.

Overall "the foreseeable harms should not outweigh the anticipated benefits" (Tricouncil, 2005, p. i.6). The benefits of this study definitely outweighed the possible harms. Scientific Rigor

No systematic rules exist for analyzing and presenting qualitative data; as a result, critics often call qualitative research "soft" (Polit \& Hungler, 1999). Therefore, methods of scientific rigor, such as member checking and investigator triangulation, are important to enhance trustworthiness of the data (Polit \& Hungler, 1999). Also, scientific rigor ensures that the analysis and interpretation of the data and present conclusions are valid and patently clear (Polit \& Hungler, 1999).

Certain steps were taken to ensure that the study's findings accurately represented the participants' perspectives and opinions. This was accomplished by including my reflection on the personal meaning of the experience and ensuring that the description 
was an accurate portrayal of such meaning (Moustakas, 1994). Secondly, "intersubjective validity" was accomplished by turning outward to those being interviewed and ensuring that the description in the findings accurately portrayed what they experienced themselves (Moustakas, 1994). This was accomplished through a member check. Three participants were asked to carefully examine the data analysis to see if it their experiences of FCC were captured in the findings. The nurses that participated in the member checks included: a nurse practitioner with extensive clinical experience and a registered nurse with little experience. The nurses involved in the member check did not identify that changes needed to be made to the data analysis; they felt it was an accurate representative of their perspective and experiences of FCC.

Confirmability is the objectivity or neutrality of data so the there is an agreement between independent people about the data's relevance or meaning (Guba \& Lincoln, 1985; Polit \& Hungler, 1999). This was accomplished through external audits. External audits were present throughout the process of this study. The supervisor for this study has experience with person-centered interviewing and a background in nursing. Both of the thesis committee members have extensive experience with qualitative research and one has a nursing background. I met regularly with my supervisor to gain assistance with the exploration, identification, and development of themes. The committee members were invited to provide constructive feedback that aided in the analysis process. This ensured that a high quality and accurate description of FCC was accounted for in the data analysis.

Transferability is essential to the ability to transfer findings to other settings or groups (Polit \& Hungler, 1999; Lincoln \& Guba, 1985). Transferability was used for this study to ensure that the data were accurate and complete. In the Discussion Chapter of 
this thesis, the findings are discussed in relation to other research studies on FCC in various nursing settings. Furthermore, transferability occurs when the reader examines the findings in light of her/his context and determines the extent to which the findings can be applied (transferred).

Dissemination Strategies

Once this research study is completed, research findings will be shared with participants, staff, nursing educators and program leaders within the Hematology, Oncology and Transplant program at the ACH. PowerPoint presentations will be provided to the nursing staff on the unit, nursing staff in the oncology clinic, and residents and physicians at Team Rounds. Also, I anticipated submitting a manuscript to a nursing journal for possible publication. 


\section{CHAPTER FOUR: FINDINGS}

In this chapter, I present the findings from the interviews of 20 pediatric oncology nurses at the Alberta Children's Hospital $(\mathrm{ACH})$. The interviews were digitally recorded and transcribed verbatim. Thematic analysis was conducted to analyze the data for this study (Polit \& Hungler, 1999). The transcripts were read carefully with an eye to identify any underlying and reoccurring concepts and patterns (Polit \& Hungler, 1999). The related concepts and patterns were grouped together to develop overarching themes (Polit \& Hungler, 1999). The data were then re-read for content and examined for goodness of fit with the themes.

The research questions that guided this study were:

1) To what extent do pediatric oncology nurses at the ACH report practicing within the FCC framework?

2) What are the barriers and challenges faced by pediatric oncology nurses at the ACH when providing FCC?

3) What practical skills and approaches are used by pediatric oncology nurses to provide FCC in the midst of barriers and challenges?

4) What strengths and capacities are associated with FCC that aid pediatric oncology nurses in providing care to children with cancer and their families?

5) How does the ACH support and encourage the implementation of FCC by nurses within the oncology program?

The concepts that were generated from the interview data were grouped into 5 major themes, and are presented in Table 4.1 . 
Table 4.1

Major Themes Extracted from Data

\begin{tabular}{cll}
\hline Theme & Sub-Theme \\
\hline 1. Does ACH & The ACH Supports FCC \\
Support FCC & The ACH Does not Support FCC \\
2. How Participants & Encompassing the Whole Family \\
Defined FCC & The Family Identified who Constituted their Members \\
3. Establishing & Collaborating with the Patient-Family: The Three Legged Race \\
FCC & Parent as the Expert \\
& Active Listening \\
& Respecting Culture and Beliefs \\
& Teamwork with Colleagues \\
& Nurse Patient-Family Relationship \\
& Letting Parents Cope in their Own Way \\
& Encouraging the Cancer Community \\
& Having the Attitude that there is Always and Opportunity \\
& Being Flexible rather than Dictating and Controlling \\
& Caring for the Parent \\
& Educating the Patient-Family \\
& Not understanding Patient and Family Culture \\
& Contradictions and Tensions between Nurses and Families \\
Charriers and & Lack of time and increasing acuity of patients \\
Providing FCC & Limited Space and Lack of Resources \\
& Lack of Education about FCC for Nurses and Health Care \\
& Providers \\
& Difficult Working Relationships \\
& Brave Face \\
& Not Passing Along Information
\end{tabular}

The participants were asked if they felt that the ACH supported FCC within their practice setting. The majority of participants $(80 \%, \mathrm{~N}=16)$ stated that "yes" the ACH supported FCC in their setting; 20\% (N=4) stated that "no" the ACH does not support FCC in their practice setting. The participants were also asked to describe what FCC meant to them. All of the participants identified that FCC meant incorporating and encompassing the whole family in the care provided to the child. The participants explained ways to establish basic FCC in their practice, as well as ways to enhance FCC 
in their practice and provide care that went above and beyond basic expectations.

Participants spent a great deal of time discussing barriers that prevented them from providing FCC. Also, it became apparent that the participants in this study faced many real and significant challenges that hindered them from providing the type of FCC they aspired to provide.

Demographic information for each participant was collected at the beginning of the interview. This information is presented in Table 4.2. 
Table 4.2

Demographic Information of Participants

\begin{tabular}{|c|c|c|}
\hline \multicolumn{3}{|l|}{ Variable } \\
\hline $\mathrm{N}$ & & 20 \\
\hline \multirow[t]{4}{*}{ Age } & $20-29$ & 10 \\
\hline & $30-39$ & 6 \\
\hline & $40-49$ & 3 \\
\hline & $50-59$ & 1 \\
\hline \multirow[t]{2}{*}{ Gender } & Male & 0 \\
\hline & Female & 20 \\
\hline \multirow[t]{6}{*}{ Education } & Diploma & 0 \\
\hline & Bachelors & 15 \\
\hline & Masters of & 2 \\
\hline & Nursing & \\
\hline & Nurse & 3 \\
\hline & Practitioner & \\
\hline \multirow[t]{4}{*}{ Number of years working as a nurse } & $0-2$ & 5 \\
\hline & $3-5$ & 4 \\
\hline & $6-10$ & 4 \\
\hline & $10+$ & 7 \\
\hline \multirow[t]{4}{*}{ Number of years working in pediatric oncology } & $0-2$ & 5 \\
\hline & $3-5$ & 5 \\
\hline & $6-10$ & 3 \\
\hline & $10+$ & 7 \\
\hline Completed a course in FCC & & 10 \\
\hline In-patient nurse & & 7 \\
\hline Out-patient nurse & & 5 \\
\hline Both In-patient AND Out-patient nurse & & 8 \\
\hline
\end{tabular}

General observations about the participants in relation to the demographic data have been formulated. A large portion of the participants $(50 \%)$ were between the ages of 20-29, all were female, and all held at least a bachelors degree. Five of the participants held a master's degree; three of whom were nurse practitioners. The years of experience 
as a nurse varied and a majority of the participants had more than seven years of experience $(\mathrm{N}=7)$. Half of the participants took a course in FCC and a majority of the participants worked in the inpatient and outpatient setting. The demographic data was examined with respect to the findings and no patterns were identified.

Throughout this section, the patient and family have been coupled into the concept 'patient-family' as a way to refer to this entity. In reference to excerpts from the transcripts, FCC means Family-Centered Care, the number following FCC is the participant study ID number, and p. is the page number from the transcript.

Does the ACH Support Family-Centered Care (FCC)?

Participants were asked if they believed that the Alberta Children's Hospital (ACH) supported FCC within their practice setting. The majority of participants (80\%, $\mathrm{N}=16$ ) stated that "yes" the ACH supported FCC and 20\% (n=4) stated that "no" the ACH did not support FCC. Overall, most of the participants felt strongly that the ACH supported FCC within their practice setting.

\section{The ACH Supports FCC}

Sixteen participants offered specific examples of how ACH supported FCC within their practice setting. Such participants observed that the institution supported and encouraged an environment of FCC through various modes, such as having FCC in the mission statement and the layout of the building.

Oh, totally. They're so good - like, I don't know. I think compared to a regular adult hospital Children's is just like leaps and bounds above where everyone else is in family centered care. And it's - even just like, you know, designing it so that each room has a parent bed in it is providing - I think that's affecting family centered care because that means that the parents can stay overnight. (FCC-13; p.2).

Yeah. I mean, I think certainly a whole group of staff that work in a Children's Hospital I think there's a premise that we're always working with the family, and the patient is as an entity... So certainly it's absolutely I think part and parcel of this 
hospital. And between trying to explain it, having it in the mission statement, and having workshops on it or whatever and - but each one of us within that I think develops one's own concept of how you're going to translate that belief. And I don't believe you could work here without having a good belief in, whichever way you want to define it, but you have to be willing to work with families when you work with children and I think within this institution and other disciplines as well you see it all the time. So I do think this institution fosters family centered care... But yes, in this institution I think we're encouraged to practice that way and I don't believe you'd be happy if you didn't or - you just can't. That's the bottom line. (FCC-04; p.4).

The participants incorporated a strong a family focus in their practice because the family was always present with the child. Those who felt the ACH supported FCC in their setting indicated that other disciplines embraced FCC as well.

Yes, very much so. No doubt in my mind. I think you wouldn't have it any other way here, especially because it's pediatrics, because you are not getting a two-year-old walking in on their own and saying, "What do I need to do here?" You have to, have to focus on the family centered care. And I know we are a multi-disciplinary team, we all focus on it as a team. There's not one person versus the other that says, 'No, I'm not focusing on the family centered care.' It's everybody from the docs to the dieticians to social work, nurses, everyone. Yeah, even the kitchen staff. Like, I think A to $\mathrm{Z}$ everybody covers it, yeah. And it's not even 100 percent; it would be a 200 percent from out point. (FCC-15; p.5).

\section{The ACH Does Not Support FCC}

Some of the participants $(20 \%, \mathrm{n}=4)$ stated that the ACH did not support FCC as well as it could or should. They recognized that the ACH identified FCC as important in caring for children and their families, but did not ensure that it was carried out to full completion. These participants observed that the ACH "talked" about FCC, but did not ensure that nursing care was structured around it. They also noted that the ACH fell short on providing adequate support and education for nurses to learn about FCC.

I think we talk about family centered care and in pediatrics we are very open about the idea that it is the child and the family, but I don't always think that - I guess I'm thinking of different things but I don't think we always go there because we often we don't always seem to respect parent's ideas and wishes. We seem to complain more than we support variation and different ideas. And I don't know if this quite fits 
but I struggle with the nursing assignments in our in-patient setting because you see a change in nurses very frequently. To me, family centered care means that you should have a good idea of who the child and the family is, the supporting units are, so when you're constantly changing like that I don't know that you can have that good idea of who you're working with as you're constantly trying to meet a new family and get to know them. So I think that lets us down in trying to do a family centered care. So I don't know, I think in ped's sometimes we talk well about it but I don't know that we always do it as well as we should... So I think we talk about it and in talking about it we are thinking we're being supportive of it in the institution, but I don't know that the structures are always there to allow us or enable us to do it. (FCC-07; p.3).

Yeah. I don't think that they really show it in a way. Like, I think that we talked about it in the general nursing orientation but I can't even remember. So I'm sure they support it but there's not - like I said, no requirements for education so there's obviously not enough funding for it for us nurses to dive right into it. So I think yes, but not show it. (FCC-12; p.2).

I think that overall I think that yes, they do in the way that maybe people don't get a good understanding of what family centered care is, you know, maybe we don't get enough opportunities to learn about it in a theoretical sense but from what I know about family centered care and what I feel we provide I think that we don't have the supports. (FCC-02; p.3).

Summary. The majority of participants (80\%) stated that the ACH supported FCC because the $\mathrm{ACH}$ has made it a priority. For example, FCC is written into the mission statement and the new hospital was built in a way that accommodates the patient-family. A few participants stated that the ACH did not support FCC because the ACH had no measures in place to ensure that FCC was being delivered to the patient-family directly.

\section{How Participants Defined FCC}

At the beginning of each interview participants were asked to describe what FCC meant to them. Every participant $(100 \%, \mathrm{~N}=20)$ stated that FCC meant including and caring for the whole family, and most of the participants indicated it was important to let the family identify who constituted their members. 


\section{Encompassing the Whole Family}

All of the participants confirmed that when they provided FCC they included the whole family. They recognized that the child was surrounded by family, therefore it made sense to include the family in the care they provided because the family was affected by the child's illness.

For me more so it's just encompassing the whole family as a patient than just the patient. So getting the whole family involved in the care, and that's just pretty much what it is to me. (FCC-12; p.1).

So, basically working in the area of oncology family centered care basically is exactly what it says: including the whole family when a child's diagnosed. Particularly in the pediatric world it's really important that it's not only the child that's diagnosed but it's the entire family. Uh, yeah, and I think that because the parents are the primary caregivers we just have to keep in mind that it's not just the illness; it's outside affects, you know, work, support, things like that. (FCC-10; p.1).

When providing FCC, the participants noted that they cared for the children in the context of their families. This was because the children were acutely ill, unable to care for themselves and dependent on their families to attend to their needs. Therefore, as a family unit, what happened to the child had an impact on the whole family.

\section{The Family Identified who Constituted their Members}

The participants identified that an important part of FCC was to determine who the child regarded or deemed to be his/her family. Families were not always traditional, i.e., a mother and father. Family was often constituted by grandparents, aunts, best friends and step parents. Whoever the family considered to be important in their lives at the time of hospitalization became a part of the child's life.

But really I guess overall it's considering the entire family, whatever the family determines the family to be, in the care of the child. (FCC-09; p.1). 
Is the family is who it says it is. So who do they consider their family to be? Cause sometimes it's maybe somebody who's not related to them, so finding out those members. (FCC-16; p.12).

Summary. All of the participants stated that FCC meant including the whole family in the care they provided to children with cancer. The children were dependent on their family; therefore, it was impossible to provide care to the children without involving their family. The patient-family determined who the members of their family were, not the health care professionals.

\section{Establishing FCC}

Participants identified specific skills and approaches they used to establish basic FCC within their practice. These included: Collaborating with the Patient-Family and Respecting Culture and Beliefs. The basic principles participants used to establish baseline FCC within their practice is discussed in this following section.

Collaborating with the Patient-Family: The Three Legged Race

\section{Patient-Family are Part of the Care Team}

Teamwork was an important feature of establishing FCC. Participants stated that it was essential to cooperate with the patient-family in a coordinated effort. The notion of teamwork and the family's place on that team fostered collaboration.

So it's all about collaboration and working together. I know that there's the multidisciplinary team but the family is included in that I think when you're making decisions. Decisions are the parent's decisions anyways so everyone has to be included and informed. (FCC-12; p.9).

Within teamwork, the idea of working with family member towards a common goal was identified as a vital aspect of care. The following participant spoke to a stressful situation where a patient had trouble with his bowel routine. The multidisciplinary 
medical team worked together with the mother to find a solution to the problem. Both the multidisciplinary medical team and the mother provided valuable input and worked together as a team towards a common goal; they helped the patient find a comfortable bowel routine.

The mother was very distressed. And just working together with her on what he needs and what the issues are, and then just bringing the resources in that I could like Child Life and the social worker and just working together as a team, including the mother. So we had meetings including the mother to decide what would be best for this child and how often meetings should occur with him between the social and him. And actually he's doing much better now, so that's a success story. (FCC-03; p.14-15).

Another participant explained teamwork within the pediatric oncology setting through the analogy of a three legged race. Her description completely summarized all of the participants' explanations of teamwork. Also, it illustrated how the nurse and patientfamily are partnered together and must work as a team towards a common goal.

I mean, the only way you're going to walk together is - like, for me children when cancer and this whole work together is kind of like when you're in a three - where it's called like a wheelbarrow race. You know where you tie your legs together in the middle? Like, your right leg and my left leg would be tied together - three-legged race. This to me is like a three-legged race. We're independent but yet we have to be attached to work together because what I can offer a family is what is sometimes beyond what they can do for themselves, whether it's they don't have the ability to absorb the information, they don't have the desire, they don't have the resources to accept the teaching and to care for something at home so we do it here. And you carry them and support them sometimes through this. And it goes the other way too. Like, they can absorb so much and be willing to take on so much, and at times guide us to say, "This is the best way to do something for my child." Right? So we are totally a three-legged race. Like, we're joined, we have to be joined in order to work together whether you like it or not. Like, sometimes you're tied and you'll put your arms around each other because you really like each other, and other times you're tied and you're like, "Dangit, I would so not want to be tied to this person but I don't have a choice. This is the family I have to work with, and they have to work with me. And let's figure out how to do it." And then it's about the communication. Who's going to move what leg when and how are we going to move the common part together? (FCC-20; p.12).

In order to work as a team the participants noted that they had to value and appreciate suggestions and input made by the patient-family. They also had to be willing 
to incorporate such suggestions and input into the care they provided. In fact, parents were identified as experts because they provided care to their child within the home setting. For example, parents mastered individual dressing change techniques at home and demonstrated such techniques within the hospital setting for nursing staff. Nurses who practiced FCC were "open" to individual modifications and did not rigidly adhere to policy and procedures as long as certain principles were met and the practice was safe, i.e., aseptic technique.

No, I think for dressings, especially when the parents have gone and done these dressings at home for a few weeks and they come back and they tell you, 'This is how I change a dressing,' then we work together with them. I mean, as long as it's safe, as long as they're adhering to policy then we'll collaborate with families and what they do and we won't dictate those types of things. (FCC-03; p.15).

\section{Offering Choices and Fostering Control}

All of the participants noted that it was important to offer the patient-family opportunities to make choices with respect to their care. Doing so enhanced their participation as decision-makers, and moreover fostered a sense of control for a diagnosis and treatment that often defied control.

I think that we all really try to, and because we get to know families so well we do make a lot of - I don't know how to say it - like for appointment times we let families, if they can have a choice we give them a choice. Like, we do - and we always try to include them in their care. The physicians I feel are really good at letting families know news from scans or where they are in their therapy. (FCC-03; p.3).

On occasion, the patient-family was not knowledgeable about the options or choices available to them. Thus, it was important for the nurse to inform them of their options and choices.

But sometimes you have kids who they're taking liquid medication or something and they want to try pills, or visa-versa. So working with the child if they want to try and change the way they're taking their medication. It would be the same medication; it 
would just be, you know, how they're taking it. And then giving them choices in terms of if you want to try pills if you want to try crushing them, if you want to try putting them in gel-caps. If there's - our Child Life Specialist is able to help with how to swallow pills, so getting that person involved. (FCC-01; p.5).

Yeah, so presenting options. Like, if there's a change that's coming up in a treatment plan which requires the families to do something I try my best to give them those choices and then let them make their decisions how they would as a family. So if the parents like to defer to the child to make the decision then that's what - I wouldn't intervene, and visa-versa as long as it's a safe thing that is happening. (FCC-01; p.5).

Choices included small things such as letting a child decide which arm was used

for a blood pressure reading. Such "little choices" were viewed by the participants as important to the patient-family. In combination, the smaller scale and larger scale choices fostered the nurses' relationships with the patient-family.

Uh, well even just a little thing. I have a little three-year-old girl as a patient today and, you know, like letting her pick the arm for her blood pressure cause I know that sometimes kids at that age really hate blood pressure. So hold it up and, 'Which arm would you like?' Like that's just a little choice for her that she can make and feel in control somewhat. I don't know. I think even going back to the building of the relationship thing, I think that the choice thing has a lot to do with that because parents are here and sometimes this is their life for months. This is their, you know, and so they need to feel like they have some sort of normalcy. And so if you let them have choices as to, you know, as much freedom as they can as to when things happen whether that be dressing changes, tasks, or baths, or going down for a test or things like that, if they have the choice of when that can happen then it makes things a lot easier for the relationship between staff and family, that's for sure. That just makes things better for them I think. (FCC-17; p.6).

Participants noted that they were not able to provide options or allow the patient-

family to make choices when situations became unsafe: certain situations restricted

choices. The following participant shed light on this type of situation:

It depends what the choice is or what the choices can be. An example would be a child comes in with fever and neutropenia, can we go home? No, and explain why and explain what the - so it depends on the situation, and of course they have choice. So you have to work within the situation itself, knowing what - it's also important to be honest with families and not presume to promise them things that you can't deliver, or try to pacify the situation if it isn't serious. If it is serious I think honesty in a caring way with support I believe in being honest with the children, the families all the time as best you can. That's just a given. So choices are always there. It might be 
a little one around this scenario and it might be, you know, you could bring this from home, but it - yeah, I think you have to tailor to the situation because you're not doing anybody a - I think it's also important not to be giving permission for everything everywhere, all the time between children needing guidance and discipline through a process like this. (FCC-04; p9).

A result of allowing the patient-family to make choices was that it gave them a sense of control. The participants noted that when a child was diagnosed with cancer the patient-families lives were completely uprooted and filled with uncertainty. One way that the participants made such situations more bearable was to allow the patient-family to have control over certain things. This gave the patient-family a sense of stability.

There's certain things that families and patients can have, take that control, cause they lose that control or most control when they come into the hospital. So there are decisions that if you don't want to have a bath at 8:00 what time works for you? And even something as simple as that that the nurses when they come in they're like, 'No, we've got to have a bath, we've got to get you up for the day.' But then when you actually talk to families they go, 'You know what? Our child takes a bath at night right before they go to bed. They sleep better,' and just knowing those little things really helps them out because then they can say, 'Okay, no you decided to do this,' so we just need to make sure that it's done at that time, and the parents are usually good at that. (FCC-08; p.8).

If that's the position that they want to be in then I think absolutely. Especially in a situation like this when they have kids that are sick with cancer because I think that's one of the biggest things is they lose control. Like, they're not in charge of everything anymore. It's not just about how they want to run things. Really the disease becomes the focus of everything and I know a lot of times they feel like they lose so much control over raising their children... I think the little things that we can put back in their hands and let them have as normal an opportunity as possible, just give them that control because absolutely I think they just feel such a loss of control over their child who's supposed to be their full responsibility and they come here and they totally lose that. So I think it's so important for us to try and give them whatever control we can. (FCC-19; p.8).

It was important to foster a sense of control for the patient-family because it gave them the determination to continue on. At times, the patient-family became overwhelmed with the cancer diagnosis and its treatment. They felt helpless. Offering the patient-family choices, thereby augmenting some sense of control, encouraged and offered hope to them. 
But that sense of control is what gets them through the next couple of days. Anything that they can kind of grasp onto, and if a child thinks, 'Well I choose to have my dressing change done in the morning and not in the afternoon,' that one thing that they're choosing can get - like, that's an instant rapport right there. It's like, 'Well you're not coming in, you're not going to bully me and say no, we need to do it right now.' And I think often times even just preparing them sometimes - it doesn't always work depending on the age of the child. You go in and say, 'Hey, you're going to get your poke at 2:00," and it's only 9:00, they're thinking about it all day. Whereas if you do have that flexibility where you can get that IM at 2:00 or you can get it before you go to bed, or what do you think?' Anything - I kind of say, 'Okay, let's just ask the child and family whether or not - do you want to try to give that med now or do you want to wait until after supper or' it's little tiny things that make a difference though because then at least the families know that, okay, I'm included in the care and I do have some say in the care. (FCC-10; p.14).

\section{Parent as the Expert}

Participants recognized that the family had a knowing and understanding of the

child that no one else had. They accorded expertise to the family because the family was

the expert who had both experience and skillful knowledge about the child.

A lot of times, I mean obviously the families know their child best and what works. I think always parents have a good sense of when their child's well and when their child isn't well. So they're the greatest asset that we can have so just always including them in everything that we do as much as we can. (FCC-01; p.13).

Since the family was the expert about the child, the participants noted that it was important to include them in the care because they are a valuable resource that guides the care of the child. The family helped the participants save time by enabling them to provide effective and efficient care to the child.

And figure out - cause a lot of times they know way better than we do if their kid should get blood while they're having a nap because it's easier, or if - you know, they know all that stuff and so I think if we don't ask them how to fit our care in with their lives, I mean, we're at a total disadvantage because they have such good perspective and an idea of how it all works. (FCC-19; p.7).

In the following excerpt, the participant described a situation where a parent knew something was wrong with her child even though the medical team's assessment showed 
that the child was fine. It was important for the medical team to recognize that the mother was the expert on her child because in the end the child was suffering life-threatening side effects of chemotherapy. If the team did not acknowledge that the mother knew her child better than anyone else and did not take action, the child would likely have died.

So I think mothers have just a built in intuition. I'm not a mom myself but just from my own mother and seeing different mothers care for their children. They just have a sense of, "My child's not right." I can remember one example. It was right before a child reacted to - we don't know what he reacted to but his lips blew up really big and even before that happened she said, "Something's not right. Something is a little off." And he reacted to - we're not sure what it was but he had a reaction and his lips blew up. So she obviously sensed that something was going to happen and it did. So just trusting that intuition or really knowing their child. I think knowing their child more than we do and, 'This behavior is not right. There's something coming like a fever or a reaction or something.'(FCC-03; p.1).

\section{Active Listening}

Active listening was identified as being important within the philosophy of FCC because it helped the participants to create supportive relationships with the patientfamily. Participants indicated that being attentive to what the patient-family said enabled them to have a more trusting relationship. When the participants listened to the patientfamily's concerns, the patient-family became more comfortable and confident voicing their opinions and concerns. This then helped them to cope better with their cancer journey.

I think probably the biggest thing is listening. Being an active listener, you know. If the family tells you something, even if - just at least acknowledging it and trying whatever their request may be, even if it's something ludicrous as listening to them and telling them you'll look into it or you'll find out. Even if I don't have the answer right away and getting back to them. Even if I have to come back to them and say, 'You know what? This is just not possible,' at least I've given them the respect by coming back and telling them that I looked into it. I think it's really important in just communicating as much as is needed so that they understand why I couldn't or whatever the situation may be. (FCC-01; p.5). 
Yeah, it gave her more - by listening to her concerns and validating them I think that gave her more confidence to be able to express her concerns to other people, you know? Cause sometimes you don't know. You think, well maybe I'm just being maybe I just am scared or I'm - maybe I just need to be braver or just figure it out on my own. But sometimes if someone listens to you and says, 'You know what? Actually that sounds really reasonable,' then you feel kind of braver to say it. (FCC02; p.7).

Participants noted there were at least two benefits arising from active listening.

First, it helped to avoid and/or manage conflict because it permitted people to voice their opinions and concerns before they escalated. And second, listening helped the participants recognize the patient-family's emotional and psychological suffering and provide holistic care.

I think for anything that comes through hospitals that listening can cut out so many problems because sometimes the way we express things and the way people understand what you've said can be different. So I think it's important if you explain something is to listen to a) their concerns cause you might be telling them something that they can't practically do for whatever reason, but getting them to feed back what you've said and listen to them. Also if you listen to people, I mean, I think - I don't think it's just family centered care, I think life, if you listen to people you discover that there's a lot more going on than what you see on the surface. And also in this environment sometimes people get so wrapped up - and I'm talking about parents, patients, and staff - get so wrapped up with the disease they forget about there's other things going on in these people's lives so you can actually sit down - if you'd spend some time speaking to people you discover that there's other stuff going on, and some of it they just need to talk about. You don't need to do anything active but it can just make their life so much better. (FCC-15; p.5).

\section{Respecting Culture and Beliefs}

The participants indicated that it was important to accept and not judge the various cultures of the patient-families that they cared for. This was done by not judging the patient-family and learning about their specific cultures and beliefs.

\section{Not Judging the Patient-Family}

Participants noted that they worked with patient-families who were from various cultural and ethnic backgrounds. As a result, it was important for them to be accepting of 
each patient-family's culture, values and beliefs. In order to provide FCC among their

patient population, the participants had to keep an open mind and not judge the patient-

families individual belief and value system.

By not being judgmental and just have an open mind I think more so than anything. I think that if you're going to come in and stereotype families because of certain beliefs and what not, like, I just don't think that nursing would be for you because you have - everyone comes from a different backgrounds, different beliefs, different religions, ethnic backgrounds. So I think that you just have to have an open mind. (FCC-12; p.3).

I think just to be open-minded. Like, I'm from a different background, different belief. Like, people sometimes might not even know where I'm from, what my background is, and for everybody to kind of like open-minded and to just not question but to support that just the need that they might have or the feeling of relief that they've done this for themselves and their son or daughter, their child, is a huge kind of support. And that's what - we just need to be supportive regardless of what our own belief system might be, regardless if we agree with it or not because not everybody's different. Everybody's different. You might think of something versus what I - you know? Like, we don't have to be on the same pattern. As far as I know that I can provide that support to them and be there for them I think that's what you need, to just approach it with an open mind and just make sure that you - you can't judge anybody. Yeah, that's one of the things with nursing I think is because we see so many different backgrounds from different ethnicity and different beliefs and whatnot, you have to have that open kind of an outlook where it's like, 'Okay, this is fine by me.' As far as the patient's safe and they're okay with it and they know it's their choice. (FCC-15; p.7).

Such patient-families made decisions and carried out activities that were different than what the participants typically encountered. However, the participants recognized that they had to respect the patient-families way of doing things so that they could continue to provide FCC.

Uh, yeah. Well, this example that I was just kind of elaborating on really. Not that it clashed with my values and beliefs but - so this family was from India and they how do I put this - so they were diagnosed, the child was diagnosed. We met with the family, went through what her diagnosis was, what the treatment option was going to be, what it entailed, and her diagnosis wasn't great; there were some things that were going to have to done surgery-wise. The family wanted a second opinion and they wanted the second opinion from a physician from India. Uh, fair enough. You know, like that's their support and they don't know us from a hole in the ground. So that was 
challenging because that delayed treatment and they did consent to certain things but not to have a central line put in, not to - and we can't go ahead with therapy if we don't have a central line, so we have to respect that and we have to, you know, 'Okay, well when you're ready come back, and if you decide then we'll put the line in and we'll start therapy.' But they wanted another opinion from a physician who was from India and although the oncologist that kind of went through what the therapy was going to be and what her diagnosis was did tell them that, you know, 'I don't want to rush you guys. I would love to say take all the time you need but I don't recommend you take too, too long just because from what we know of this disease the longer we wait the harder it's going to be to treat it and she may metastasize somewhere else.' So he did - and we were all in there. We were very supportive. (FCC-10; p.12).

\section{Learning about Specific Cultures and Beliefs}

The participants had to actively engage with the patient-family in order to learn about their specific culture and beliefs. They noted it was important not to make assumptions about the family's culture, nor make assumptions based on skin color (race). Also, the participants understood that there were cultures within cultures. For example, within the East Indian culture each person can have their individual set of beliefs and values.

Participants learned about the each individual patient-family's culture and beliefs by engaging in a conversation and creating a relationship with them. By acquiring knowledge directly from the patient-family rather than from other sources, i.e., textbooks and cultural material, the participants were better informed and equipped to provide care that was culturally appropriate.

I think that's another thing we do a really good job of. I think we don't necessarily sit down - like, they have that silly form. You know, you admit them and it says, 'What are your beliefs?' We don't sort of - I don't typically - that's not when I get to know my families and that's not when they're about to divulge all their beliefs and culture. It's through the relationship that you build with them, so again, asking the right questions at the right time and just be engaging the families, learning more about the families. And it's not just about different ethnicities. You can have Caucasian people with all sorts of culture and tradition and things, or beliefs and stuff. So I think that we respect that by the fact that we do engage our families and we do get to know them. (FCC-16; p.9). 
I think that if I'm not aware, like I'm not used to - what it's called - Hutterites. I'm not really that aware of their culture or of Native Canadians. I'm not sure of - and that's not so much a culture for the Native Canadians but I'm not aware of what rights cause they have different rights from other people, so I would try and find out either by just speaking to family cause I don't think most people are offended if you're quite open and say, 'I'm not from here. Can you explain?' So I would ask them to explain if there's anything that they feel that I could do to make it better for them. I find out if they're on a special diet, that type of thing, for some people that males are not to go in a room unless - because the mother has got to wear some sort of head covering. You know, to knock and make sure that there's a sign available. If it was something to do - so it depends what it is, so I would try and listen and find out when I'm doing an admission or in the role that I'm in find out what the values are so I can pass them on. (FCC-11; p.6).

The following participant expressed the idea of not making assumptions about culture based on a textbook definition; that one must ask the family directly in order to acquire such knowledge.

I think the biggest thing is just to be sensitive to that individual family cause when I was an educator it was hard to teach - cause there are resources out there that this culture does this or values this or has beliefs on this, but if you don't actually talk to the family then you're not respecting them as a family. Cause often times we see families and we're like, “Oh, well they're whatever religion, or they're from whatever background. They must be exactly like the family we had last week." And if we don't talk to them often times we miss that, well no the family we had next week just immigrated here so they have different values. This family has lived here all their lives, like they were born in Canada so their values, beliefs are different. Or they're from different regions. And I think sometimes we clump people together and just assume so I think it's very important to talk to each individual family just to try to be sensitive to what is most important to them and not assume. So I think that's the biggest thing. (FCC-08; p.7).

Summary. As discussed, the participants identified basic or fundamental methods that enabled them to incorporate FCC into their practice. Collaborating with the patientfamily was vital to the provision of FCC because it was important to work as a team with all parties when providing care to children with cancer. It was beneficial to include the patient-family in the decision making because they were the expert on the child. A majority of the patient-families came from various cultural and ethnic backgrounds; 
therefore, accepting and learning about their individual culture and beliefs was fundamental when providing FCC.

\section{Enhancing FCC}

All of the participants were aware of the importance of FCC and recognized that it was a part of their everyday nursing practice. They noted techniques and approaches that enhanced FCC within their specialty that went above and beyond merely establishing it within their practice. Teamwork with colleagues, the nurse patient-family relationship, letting parents cope in their own way, encouraging the cancer community, having the attitude that there is always an opportunity, being flexible rather than dictating and controlling, caring for the parent and educating the patient-family were all ways that the participants enhanced the provision of exceptional FCC within their practice. These techniques helped the participants go above and beyond basic FCC; to strive for excellence in practice.

\section{Teamwork with Colleagues}

\section{Importance of Teamwork with Colleagues}

Working as a team with various disciplines enhanced FCC. Participants relied on and embraced various disciplines because they knew that they could not do everything on their own. When the participants interlinked their care with other disciplines, their workload was lighter and they were able to provide better care to each patient-family in their assignment. They recognized that persons from different disciplines encompassed valuable strengths that they brought to the table.

Yeah, absolutely. And I think we're really lucky to have such a comprehensive multidisciplinary team here and that we have all those different disciplines and we can talk 
to them and consult them and work side-by-side so that the families get the best care that reflects their needs. (FCC-19; p.6).

Well, and I mean that's half the battle. Like, I mean without a good team and without people that are, like, okay, like this is what we're here for, this is my job and we all have different job descriptions but we all interlink with each other to make it go better. So yeah, I think having a team that you know supports you and has your back is half the battle. I mean, it just makes your life so much easier. (FCC-10; p.8).

Each discipline within the team recognized they were working towards a common goal. Therefore, members of the team were willing to help and support each other. When one person felt overwhelmed, members of the team stepped in to lighten the load. This helped the participants accomplish their work and enhance FCC within their practice.

And I mean, you do rely a lot on your co-workers, right? Like, I mean we relieve each other, there's three primary's for the general oncology population, and I mean the dayroom girls are great. Like, we're all here, again, for the same reasons. So often times if - I mean, I'm only one person and I wish I could somehow - some days I just want to cut myself in six pieces so I can just be a part of everything, but you know we're all here together. So often times, you know, if I know that my colleague is sinking and is overwhelmed then I'll go out and talk to one of the families... But that's the thing is you just kind of - you delegate a little bit and the dayroom nurses are great. If they can answer the questions for them they do it and then they update me. And if they know that I can't come out then they have my back and they're like, 'You know something? This is what's going on with the family,' and if they can answer their questions and if they feel like, 'Okay, yeah, they seemed pretty confident when they left, I think they're good.' If they don't then they'll page me and say, '*****, you know something? I think you really need to come down here and see this family.' (FCC-10; p.7).

\section{Social Work, Child Life, Physicians and Spiritual Care}

Child life specialists, social work, physicians and spiritual care were recognized as particular team members that were valuable in the provision of FCC.

Child life specialists. Participants recognized the importance of offering the children opportunities to play and have fun. Unfortunately, the participants did not have the time to play with the children because their nursing care took precedence. Therefore, child life specialists were valuable to the participants because they were able to play with 
and entertain the children when the participants were too busy. They had a special skill set and knowledge that helped the children escape the hospital and enter a world of fun and play, which was beneficial to their overall well-being.

Uh, I think Child Life is really good at doing that kind of stuff. Like, there is - again, the same boy that was palliative, Child Life came in and got to do painting handprints and stuff and his brothers got involved and - I thought that was so cool just - it's nice to have resources like that that can come help even when we're really busy and they can come help and do those little extras that we don't always have time to do. Even though I want to be a part of that part too [laugh] but yeah, I think they're a good resource to have for sure. (FCC-18; p.5).

Social workers. Social workers were noted to be an important members of the team. Social workers provided support for the patient-families and worked in conjunction with the child life specialists.

And with social workers we include the siblings or the grandparents, and Child Life is really good at including the siblings even cause that's tricky, you know. If you have a younger brother or sister or an older brother or sister who all of a sudden they're diagnosed with cancer they are a priority, and the little kids, the siblings, get put on the back burner. But Child Life is kind of always there. And social workers are great with, you know, story books or toys to go through with them. (FCC-10; p.4).

Physicians. There were a group of physicians who worked primarily in pediatric oncology at the ACH that participants had a good working relationship with. Participants were the eyes and ears for the physicians, and it was obvious that the professions worked well as a team to provide FCC to patient-families. The participant below explained a situation in which the nurses and physicians worked well as a team.

I can't tell you how many times that this does happen where a parent will just say, or a child will say, 'I don't know exactly what's going on, just something seems wrong.' They can't put their finger on it and I'll call our bedside physicians and I'll say, 'Everything's stable on paper but we just, either nursing gut or family gut, we think something is not right,' and the physicians don't question. They just come right on down and do an assessment and we start looking deeper into things. They might start doing blood work or things that we wouldn't typically do at that time and then low 
and behold we find something that's going wrong. So it's really interesting. (FCC-16; p.9).

Spiritual care. Spiritual care providers were noted by the participants as important members of their team. They were accessible, available and open to various religions that the patient-family practiced. Also, they were accessible for the participants themselves if they had any religious or spiritual questions. Spiritual care providers were found to be a valuable part of the team because the participants noted that spirituality was an important factor in oncology nursing.

Especially the spiritual care services that we have here, they're so easy-going and they're so - they're almost very open that I think that they don't push themselves on anybody but I think they are very helpful to certain families. And if a family doesn't feel they need them or want them they don't bother them. But a lot of families, no matter what their religion is, I think that they can rely on a lot on spiritual care. And so I think it does promote family centered care to have that service available because there are a lot of people with spirituality, and when you're in times of distress which being in the hospital is very stressing and, then a lot of people might really need that and we can offer that. And I think nurses feel very comfortable just to pick up the phone and call them because they're very friendly people and they actually are very present. They come down all the time just to play songs for the nurses on their guitar or - you know, people know their names and so you can call them up on the phone and say, 'I think this family would like to see you.' (FCC-02; p.12).

Overall, the participants stated that the disciplines within pediatric oncology at the $\mathrm{ACH}$ worked well together, and as a result, FCC was enhanced.

\section{Nurse Patient-Family Relationship}

The nurse patient-family relationship was an important aspect of FCC. This special connection allowed FCC to be effectively implemented into the participants' nursing practice. In contrast, when the nurse patient-family relationship was absent or limited, it became difficult to implement FCC. 


\section{Fostering Comfort and Tailoring Nursing Care}

It was important to ensure that the patient-family felt comfortable during their stay in the hospital. Participants tried to promote an environment that was comfortable and enabled the patient-family to feel "at home." They accomplished this by being approachable and showing the patient-family around the unit. In addition, the participants recognized that the hospital was the patient-family's home during their cancer journey. Therefore, they adapted and altered their care to make the hospital comfortable for the patient-family. A specific approach used by the participants was to be considerate and tailor the care to the patient-family's needs and wants.

Like, we have the nursing station kind of central, the hallways coming off of it, and I know families feel really comfortable approaching the station and every always does what they can so that people feel comfortable to do that. We really encourage on the unit for families to - we show them where things are in the kitchen and where certain supplies are, where they can go and get them to encourage helping themselves and feeling at home. (FCC-03; p.8).

It's like a mom said to use, it's that making sure a pump doesn't beep during the night. How many times do I hear that in the morning after a nightshift. Oh, the nurse was great last night, she didn't let the pump beep all night. Well, this is their home, this is their home away from home, and we have a family on our unit that said they feel this is their home when they leave here. That's huge. (FCC-06; p.5).

Like I said before, you know, most often on mornings I'll go in the room and just be like, "Alright, what's the plan for the day?" You know, like, "This is what I need to do. What do you -" cause maybe I don't know that the kid hasn't had a bath in three days and that's what mom really wants to get done or whatever. So that's kind of what it looks like for me. I try to - for me it would be outlining kind of a plan with the families at the start of the shift. Or if something else comes up trying to give them an opportunity to time things or schedule things. I think that's a big thing, you know, cause the families feel like their time is so kind of taken from them already with having to be in the hospital. So if they can try to have things scheduled, you know, then they really appreciate that. (FCC-17; p.11).

Fostering comfort and tailoring care enhanced the nurse patient-family relationship because it demonstrated that the participants truly cared for the patient- 
family. Also, it illustrated that the participants wanted to provide comfort care given the hardship and suffering encountered by the patient-family on their cancer journey.

The participants indicated that it was important to get to know each patient-family individually. It was valuable to pay attention to personal details, such as their preferred hobbies and favorite activities. By doing so, the participants were able to include such things into their care and provide care that was individualized and tailored to each patient-family. With an understanding of the patient-family's uniqueness, occasionally the participants were able to make special events happen.

I think just getting to know your patient and your family, like what do they like to do as a family on the weekend? Do they have pets or is grandma and grandpa involved? Are they sports family? Do they like traveling or did they in the past? What did they do as a family unit? Maybe mom and dad aren't together so how does that work for when they come into clinic or when they come into the unit? Who's who and who's actually taking - who's got that active role in their life? And peers and their friends that's huge. And just asking them about school. I mean, you have to remember they might be in a hospital bed or a clinic bed but at the end of the day they're still like an eight-year-old patient who has a life outside of our clinic. You want them to have that life outside of clinic and outside of being a patient. Maybe bringing little bits of that in with them. When they have a special event sometimes they'll bring in photos and things like that. That's important I think. (FCC-14; p.9).

One of our patients was in palliative care and he really, really, really wanted to go to Toys R Us and we knew that he only had a couple days probably and so I kind of got that - like, mom was kind of talking about it and it's like, 'Oh, how can we get him to Toys R Us,' you know, and trying to figure out how we can make that - so I talked to Child Life and Social Work and stuff like that and then we kind of ended up kind of making Toys R Us in his hospital room cause he couldn't get there. (FCC-17; p.1).

Enabling special events and outings to occur made a positive difference in the well-being of the children. It permitted them to play and be children; which was frequently lost while hospitalized. Special events and outings had a positive effect on the children's emotional well-being, which in turn impacted their overall health.

And if I can bump things around for them or antibiotics every day so we can have a longer pass, because that's important. Like, you don't think passes are important but I 
can't even count the number of times - just last week a kid comes back from a pass. 'How was the pass?' and the mom says, 'This is a different kid. He's not even the same kid.' Well, what affect does that have on the rest of the body and treatment and healing and how that kid's going to tolerate the rest of the science stuff we give, right? That psychosocial piece is just as important as far as overall health. So we bump those antibiotics a little bit this way and a little bit this way to make that window for a pass bigger if we can, right? So yeah. (FCC-16; p.1).

\section{Small Things make a Big Difference}

The participants noted that even though they were busy, there were small things that they could do that made a big difference for the patient-family. Doing small things, like preventing an IV pump from beeping and shutting the door when they left a room, helped the patient-family to get a better night's sleep.

Or like when the parents are really tired and they just ask you to be quiet that night, so I'll set my pump - like, I'll go in before they're - like, the pumps beep so that they don't have to they don't have to wake up 100 times during the night and call. Or, like, shutting the door when you go in just so that they don't have to hear all the bells outside in the middle of the night and everything else. Sometimes, yeah, it's just nice for them I guess. (FCC-13; p.4).

Getting a warm blanket and turning down the lights helped to make the patient-

family more comfortable during their hospital stay.

So, to turn to mom and say, 'Would you like me to get a warm blanket for her? Would you like me to get a pillow for her? Let's dim the lights a little bit too until the doctor comes in so that she can be more comfortable and her head doesn't hurt as much.' And that's - like again, you're taking care of the family because mom wants so hard to comfort her and she hasn't got the tools that she needs to do it. So I can then bring that in and I don't need to be any kind of hero at all but to give it to mom and say, 'Here you go,' so that then she can snuggle her little girl and she can put the pillow under her head and she can put the warm blanket on. (FCC-20; p.2).

The participants noted that doing small things made a big difference to the patient-family. They stated that sometimes they were too busy to do what they would ideally like too, but there was always the opportunity to do small things, which in the end truly made a big difference. 


\section{Nursing Presence and According Personhood to Patient-Families}

Participants noted that being present was comforting and healing for the patientfamily. It allowed the patient-family to open up, cry and be vulnerable. As a result of nursing presence, they never felt alone in their grief and sorrow and knew that they had another person to lean on for support. The participants shared that being present was like giving the patient-family a gift. Being present helped the patient-family feel valued and important, which fostered a relationship between them.

There was one little boy we had, I think it was back in January, and he actually passed away at the end of January but at the beginning when things were noticeably starting to go downhill a little bit he'd gone down for a test and I walked into his just to check in and see how his mom was doing and she was doing a puzzle and she was just bawling. And so it was - yeah, it was good to just have some time while he wasn't there because she could be really honest about how scared she was and how it was - yeah, I think it was a free time for her to just really share because he wasn't there, he wasn't even in the room or anything. And it was hard to sit there and listen to that because I - I still don't know what I would have said. I haven't thought of the right words but I think just sitting there with her was okay and crying with her I think, yeah. (FCC-18; p.5).

I think just to kind of - sometimes it's just the silent communication too. Just them knowing that you're there. (FCC-15; p.7).

I think it all goes back to that thing I said, that it's a gift. That being able to..okay like it's running in your mind, you got IV, you got blood, you got.. I just keep saying the same things.. but you got that going on in your mind, I've got these things to do, but if your like, they can wait because they can, because they can, and you can be really present, especially when they are telling you a story about their kid, you know they just want you to listen and laugh, and you know, and if you're not, they can tell when you're not real, you know. (FCC-06; p.5).

View the Patient-Family as Human Beings

In order to further a relationship with the patient-family the participants noted that they had to view the recipient of their care as a human being and not just a patient. 
Like, for me I guess it just comes really naturally and I think that that's the whole key is just they're people; they're not just patients I guess is the way I think of it, you know. (FCC-17; p.3).

If we don't get involved and we don't see it as humans, then we're just doing tasks and we are just obeying orders and we're not practicing our registering nursing, you know, the whole part of our role. (FCC-06; p.4).

By viewing the patient as a human being, the participants were able to care for their emotional, spiritual and mental domains. This permitted them to understand how patient-family was truly doing and coping with their situation. Also, it solidified their presence with the patient-family.

I think it is important when you start your shift or when you're in clinic and reassessing someone that it's not just the physical assessments that you have. It's also taking into account the emotional needs, the emotional assessment of the family of the patients, the spiritual needs and things like that so that if you physically think everything's fine that's not complete, that's not enough. You need to look and see the rest of the picture, like mentally how are they doing? Are they managing? Are they coping? And if not being the one to identify where they need help and hopefully contacting the right other member of the health care team to support them. (FCC-14; p.3).

A holistic view of the patient-family enabled the participants to engage in FCC. Science and research were no longer the participants only focus when they viewed the patient-family holistically; the patient-family's psychosocial well-being was included in the care. Related to this holistic view, the participants recognized that enduring childhood cancer was stressful and emotionally taxing on the patient-family. Caring for the patient-family's emotional well-being necessitate that the participants had to consider the human/holistic aspect of the patient-family and not just the physical.

Uh - well just, yeah, it just really is important and we just - I'm a science person, I come from a science background. And then doctors, nurses, respiratory, we all come from that science background and so we put a lot of validity and truth to the numbers and the data that we collect. But there is this other piece, the mystery of what we do and the mystery of the outcomes so what influences that? So then there's this other piece in the human, the psychosocial, the belief, mood, you know, all those other 
things do impact health. And research does show it but we don't talk about it as much, do you know what I mean? So it is just important. It's just harder to tease out that cause and effect but I do believe there is a cause and effect. So I think that is in part part of the reason why this - exactly what we're talking about actually, that family centered care is important because it not only helps with compliance of the treatments for sure but it also helps with that psychosocial [inaudible - pos 6.30] how the child is tolerating therapy, how is the family tolerating therapy, and their extended support network, how is everybody sort of doing, you know. (FCC-16; p.2).

I think a lot of it has to do with taking care of more than just the physical part too and making sure that they're doing okay emotionally, cause I think especially in a center like this they're going to be - emotions are pretty on edge I think. Yeah, making sure that you're taking care of that part, not just taking care of their child's physical health but kind of seeing the whole picture. (FCC-18; p.1).

Viewing the patient as a human being enabled the participants to provide FCC that was holistic in nature; focusing on the patient-family's emotional, mental and spiritual well-being comprised this holistic approach to caring.

And finally, "time" factored into the nurse patient-family relationship. As a result of spending extended periods of time together, the nurse and the patient-family established a relationship. Because they journeyed through difficult times together, strong emotional connections and bonds were made.

Because of the nature of our setting we develop such strong relationships with families because we deal with them for sometimes three years on treatment. (FCC-04; p.15).

As with our families we see them from diagnosis to discharge, which could be two years from discharge, from active treatment. And then sometimes they come back for relapses, so it just never - and even with our population we talked about - I think to the security officer, he couldn't figure out why people do this but we were telling him that bereaved parents come back because it's closure, they want - because we're such a big part of their family, they actually call us part of their family, that they come back to the hospital to visit or for coffee or for pizza night, and nobody else in the hospital can figure out why parents would come back. But when you spend two years with people caring for your child then that bond forms and it's just hard. So we have bereaved parents coming in, we have parents that are being discharged. So I think it's the whole emotion that causes the most stress on the unit. (FCC-08; p.8). 


\section{Outcomes Associated with the Nurse Patient-Family Relationship}

When participants entered into a relationship with the patient-family they experienced satisfaction within their role. Being in relationship made them feel as though they were of value to the patient-family. When participants made a difference in the lives of the patient-family, they not only felt appreciated but they enjoyed their profession/role more.

So those are the kinds - when you see a relationship building and to me you're - as a family they're also telling you that they feel like you've got a role with them in this process, and to me that's all I need to keep going and to have the strength and more or less the courage to carry on when things are difficult. (FCC-04; p.3).

And that's, I think, to me - it was the greatest feeling to be able to know that not only have I, as a nurse, made a difference but as a human being kind of accomplished that level of comfort for somebody for them to be able to trust me so well with their child and with them that it was one of my best experiences I think. (FCC-15; p.2).

The participants indicated that their job was stressful at times. However, when they knew that they made a difference in the lives of patient-family they gained strength to continue on.

But I think, for myself, like there's days that I hated my job, that I felt like I couldn't do anymore for a family or for a child. But then the next day a patient comes in that you haven't seen for 10 years and is doing great and getting married and gives you a hug, and you're like, 'Yeah, that's why I'm here.'(FCC-08; p.4).

\section{Letting Parents Cope in their Own Way}

The participants identified that each family coped with their child's illness differently and that it was important to let them cope in their own specific way. Some families tried to do everything they could to keep their child alive, and the participants stated that it was important to just let them do what they needed to do to deal with their situation and find peace. Since each family coped with their situation differently, the 
participants observed that it was important for the nurse to identify where the family "was at" and support them appropriately on their journey.

You have to respect their wishes. Like, if that's what they want and that's what they want. Uh, I have my own beliefs about patients dying, like kids dying. I have my own way of looking at it, but I can't put that on the parents. Like I can't be like, 'yeah, your kid's dying.' No. if they think that this naturopath medicine's going to save their life then they can do that but it doesn't hinder my care. Like, they're the ones mixing it into his feeds. I'm the one that's going to do the rest of the care and do his blood work and therefore do his vitals. So yeah, I can't - I just respect it. You can't bestow anthing else on them, you can't tell them a way to think or a way to do. (FCC-12; p.6).

Or like, when a kid is dying just respecting that the parents need time to kind of cope with it and just like, there's all those stages of grieving and their spirituality is part of that. So just respecting what stage they're in and helping them cope with that is a big part of it. (FCC-13; p.8).

Occasionally, the participants did not agree with the decisions that the family

made for their child. However, they recognized that at times the family's decisions were ways in which they coped with their situation. Even though the participants did not agree with the decisions, it was important for them to let the parents handle the situation in their own way to help them cope.

But I think parents genuinely want to do good for their child and I've been in situations where parents have just said, you know, 'My child's been through this, I know he's relapsed but no more treatment.' Like, no more pain, no more - which is hard for the medical profession to digest just because we're like, 'Well, but there's this study and this study. You might be able to have a couple more months or whatever.' Right? Like, we're always kind of going for - but I think it's very - you have to respect what the families want and really, I think, respect them as families to actually identify that you know what, we don't want him to suffer anymore. (FCC-08; p.15).

\section{Encouraging the Cancer Community}

The participants noted that an important part of FCC was enabling the patientfamilies to form a community of networking and support among themselves. The cancer 
community was a source of strength and encouragement that the patient-families used

because they shared common interests, understanding and experiences.

Because that's another definition of family too is all of these families of moms and dads and kids are a family of cancer culture or community and they share experiences and they support each other. (FCC-20; p.3).

Specific activities that brought the families together, such as pizza night and cancer camps, enhanced FCC because the cancer community became an extended family. These families shared a special bond, and spending time with each other helped to make each of their journeys more bearable.

Well, I guess we have a group of parents that come on Wednesday nights and have a pizza night for our families, which I think helps to provide family centered care in a way that they get to meet parents of kids that are currently in the hospital meet families that know their child has been through treatment and they've come back to try and give back. So they meet families that way. And I think seeing other families that have gone through, it helps them. (FCC-01; p.2).

And if they want to stay in the city, if they feel more comfortable staying in the city we give them that choice. If a child is ready -and often times we kind of hit this where they get comfortable at Ronald McDonald House. I mean, that facility is fantastic and it's like their own little community and their own little family over there, and often times their child may be well enough to go home. Like, 'You can go home to Lethbridge,' and they kind of just, 'Well no, I'm okay staying here. My little family and I'm a minute from you guys and - ' And if they can stay, you know, if you know they're coming in in a day, okay, sure. 'You're coming in in two days? Okay, fine, you can stay.' (FCC-19; p.14).

\section{Having the Attitude that there is Always an Opportunity}

The participants stated that it was important to have the mindset or belief that there is always an opportunity to provide FCC. It was a matter of having your eyes and ears open for the chance.

So I guess it's important to kind of know I think there's always an opportunity to attempt family centered care but you've got to gear your queues both for timing, where the family is at, how they see you. (FCC-04; p.2). 
The participants indicated that no matter how busy they were, there was always a chance to do something small to include and consider the family. It was vital to have the attitude that there was always a chance to provide some degree of FCC.

Uh, well the only thing I can think of is time, that time is always an issue. But then on the other hand I think there's always time for family centered care. Like, we talked about the degrees before. Even just a quick phone call to a family at home or whatever just to keep them up to date on things, which takes two minutes, and - like, I think it can be done but I think time is used as an excuse a lot. So I don't think there should be anything that hinders some degree of family centered care anyways. (FCC08; p.5).

The participants noted that pediatric oncology nursing was a specialty where they had the opportunity to provide excellent FCC because of the long term relationships they formed with the patient-family. Therefore, some participants felt that there was no excuse for not providing some level of FCC in their practice. Recognizing this idea was a way to enhance the provision of FCC.

I think pediatric oncology nurses have a really good opportunity to provide family centered care, just the way it's set up. And I think that they have opportunity to be really good at it if they want to. (FCC-02; p.18).

\section{Being Flexible Rather than Dictating and Controlling}

Being flexible was a valuable way to enhance FCC. The participants indicated that it was beneficial to take a caring approach that was adaptable and subject to change rather than being rigid and inflexible. Flexibility included being open to negotiate what was best for the patient-family and a having a willingness to compromise when events would happen. By doing so, the patient-family was more satisfied with care, which in return made the nurse's job easier and more enjoyable.

It doesn't make a difference to us really, if we take our break half an hour later with the dressing change, why not.. why not. I'm not talking about sacrificing your break 
time, we need that. But I am saying why can't we be flexible, because that is family centered care, flexibility. (FCC-06; p. 5).

If it's something that needs to be done then I will try and compromise the best way. I would explain why it needed to be done the way it was being done; I would try to find some sort of compromise. If it was something that didn't need to be done then I'm not going to force - I would never force - or if it's something that can wait until later and it's. (FCC-11; p.5).

The participant below explained how she incorporated flexibility in her practice as a way to enhance FCC.

Uh, so for an outpatient let's say, often times if they have scans and things the same day as their physical exam we try to coordinate that. So if a family says, you know, if they're trying to still go to work and try to cut costs with sitters and things like that we do try to accommodate. Like, okay, if you can only come in on one day we do our very best to make all the scans booked the same day and then have their physical exam in the afternoon. If that can't be done for some reason then we ask them, 'Okay, you can come back for your physical exam in a couple of weeks if that works okay with you as long as the kid is okay and no symptoms are worrisome.' But for the most part, yeah, we kind of - we go with how they're feeling. And if for some reason they are having a hard time making certain appointments cause often times it's difficult to fit everything in in one day, but we actually write them on the requisition if they're from out of town. And if you can try to coordinate a GFR, CT, a bone scan all in the same day that would be greatly appreciated. And often times because our oncologists have certain days, so if each day there's a different oncologist that's a primary for each day of the week, so if a family can't come on their primary oncologist's day which we strongly recommend that they do. And if it's not a good reason, and when I say that it's not a good, 'Oh just because Friday's work better because of school,' we actually - if it's a really good reason and if they are living from out of town then we actually call the primary oncologist and they have coordinated and they're aware and they actually make time at the end of the day to come to not necessarily their clinic but to come to assess their patient if it makes it easier for them. So yeah. (FCC-10; p.1-2).

\section{Caring for the Parent}

The participants indicated that FCC included the responsibility of ensuring that the whole family was cared for. At times, parents became so focused on their child that they forgot to care for themselves. Parents were at risk of health concerns or other various problems when they did not care for themselves. For example, a mother became 
exhausted, dehydrated and malnourished because she did not eat, sleep or care for herself;

she only cared for her child. Participants voiced that it was the nurse's responsibility to

ensure that the family members were taken care.

I think by the time he passed away she was so exhausted herself that I think potentially she may have regretted how she was. But that's just me, I don't know that. Yeah, it was really, really challenging to watch her trying to be with him when really she just really wasn't there because she wasn't taking care of herself at all. (FCC-01; p.7).

I think at times again we get focused on the patient in the bed but if you don't take care of the whole family then that's going to impact the patient in the bed too. So if the mom's really stressed or anything that's going to impact how she cares or interacts with the child or the nurses on the unit and how care is going to be given. So I think it's very important to talk with parents, to make sure they're sleeping, make sure if they're not sleeping arrange a volunteer to come in in the daytime so that they can go take a nap, make sure they're eating, make sure they have the resources. Sometimes parents come in and nobody's working and there are resources available for food cards, for accommodation, for things like that. And asking, like asking about siblings cause often times those are the ones that they're worried about. They're worried about their child in the hospital but their child has doctors, nurses, social workers - everyone looking after them. But then they have other kids that are out there in school and trying to deal with things being maybe left from home and living with an aunt or an uncle to care for them. (FCC-08; p.6).

If the parents were healthy and cared for, then they were able to care for their child and be valuable members of the care team. As a result, the health and well-being of the child was enhanced and the participant's work load was lighter.

Well, yeah, and like if the parents are happy the kids are happy and the parents are less stressed then the less stress and - it makes our job easier too cause if the kid is crying and the parents are freaking out because they've had no food and no sleep and no water all day or whatever, then it makes us busier because we have to come in and kind of do that extra support. Whereas if the parents are happy and fed and - I don't know, to me being fed makes a big difference. (FCC-13; p.8). 


\section{Educating the Patient-Family}

It was recognized that within the specialty of pediatric oncology, the patientfamily must learn about the diagnosis and monitoring and treatment of the disease. Therefore, educating the patient-family about such things was vital to enhance FCC.

\section{Different Stages and Types of Learning}

The participants noted that there were different stages throughout the treatment continuum that required various depths and types of education. It was important for the participants to understand the different points along the treatment continuum and direct the patient-family's education accordingly.

So, depending on where the family is at, cause I think a different - along sort of a cancer treatment continuum they're going to be at different stages where they'll want certain information. (FCC-01; p.9).

So if they're the kind of people who just want to sit down and chat about it then I'm happy to do that. If they're the kind of people who like to read and gain information I just make sure that they go to places that they're getting good information - again, listening to them and taking queues from them to what would be the best way for them to get information and how I can be of help. (FCC-01; p.9).

Each patient-family had a different style of learning. Some wanted nurses to sit down and explain everything; some wanted nurses to provide educational handouts; and some wanted directions to Internet websites. It was important for the participants to understand how each patient-family learned so that they could appropriately inform them accordingly.

Some families wanted to know everything, where as some families did not care as much and just wanted the nurses to focus their energy on providing care. Therefore, an important part of educating the patient-family was learning what their priority was and adjusting the education accordingly. 
And so it would really be strategizing with the family at that point to say, 'What is it that you need?' And from that you would get a whole range of things from, 'Tell me everything,' to, 'Tell me noting. Tell it to my friend. She's going to keep track of the details.' Sometimes the families would just look at you with a blank stare saying, 'I don't know what I need.' They just start completely blank, and so that's when I would maybe get a little more suggestive in terms of what I've found helpful in the past with other families is, 'Have a really close friend or a family member in the room with you for the meetings with the doctor to write everything down so that they can be the note taker and you can be the listener. Would that be something that would work for you?' Using those kinds of strategies. But really, I'd say that's where the art of nursing comes in is to find out what approach works best for each family in that time. (FCC-09; p.12).

\section{Education Enables and Empowers}

The participants stated that education was essential in enhancing FCC because it empowered the patient-family. When the patient-family was well educated they were able to be involved in care and decision making, and were better equipped to ask questions of the medical staff. Education gave them the ability to be more independent and not rely solely on the medical staff.

And I think education is like number one because if they are educated then they can ask the questions and have the knowledge to be involved. Whereas if they know nothing they're just going to kind of sit back and keep them dumb and then they don't know. (FCC-02; p.15).

I think it's all about education in your family centered care. If you are making sure that they are - education is power. As much as they know, as much as we inform them is what they're going to go home with when they are going home. And when they are on their own that's what's going to come to them. We're not always going to be here. So that's, I think, giving that tool to the family is huge on our part or huge in the family centered care, which plays - I think it impacts the whole system whereas if we didn't give that education we're taking basically everything away from them and they're going to feel vulnerable, they're not going to be able to be independent, they're going to feel the need for us to be a 24-hour kind of assistance to them, which I think we encourage to get away from that. So to inform them with everything, good or bad, and to make sure that the family's comfortable I think plays a huge role in family centered care. (FCC-15; p.9).

The patient-family eventually went home from the hospital and had to care for the child without medical assistance. Without education, going home was not safe because 
they were vulnerable. Education was seen as a tool that the patient-family utilized to be an independent unit and care for themselves.

Summary. Teamwork with colleagues and fostering the nurse patient-family relationship were methods that participants used to enhance FCC within their practice. These approaches enabled them to provide holistic care. Creating a relationship with the patient-family enhanced FCC because it ensured that the patient-family was comfortable and the care provided was tailored to meet their individual needs. Education also enhanced FCC because it allowed the patient-family to become more knowledgeable and empowered them to be more involved in the care.

\section{Barriers and Challenges to Providing FCC}

During the interviews, the participants discussed and described barriers that hindered them from providing FCC to the patient-family. Factors that undermined the participants' ability to provide FCC have been grouped into the following subthemes: not understanding patient and family's culture; contradictions and tensions between nurses and families; lack of time and increasing acuity of patients; limited space and lack of resources; lack of education about FCC; difficult working relationships; brave face; and not passing along information. These themes are discussed further below.

\section{Not Understanding Patient-Family's Culture}

The most commonly mentioned barrier to providing FCC was the lack of understanding and knowledge regarding the patient-family’s culture. Many patients within the oncology department had different cultures than the participants. Calgary is a growing, multicultural city. Therefore, many patient-families had cultures that were unfamiliar to the participants. When the participants did not understand the culture of the 
patient-family, they were unable to fully provide FCC as they hoped. The following quotes illustrate this point:

I think with the amount of ethnic groups that we have coming through Calgary and coming through clinic I don't have a good appreciation of what their definition of family is. I don't have a good idea of what their culture and faith is that defines their own community and family, so I don't know necessarily how to support them if I don't know what their traditional roles are as mother and father or husband and wife. (FCC-20; p.4).

But I think it's hard because I don't have a lot of knowledge of different religions and ethnic backgrounds and I don't know a lot of their ways around things. (FCC-12; p.4).

The participants shared that occasionally patient-families had cultures that encompassed certain religious customs and rituals. Often times, the participants were unaware and oblivious to such customs and rituals. For example, one participant shared that while a patient was dying, she was in the room trying to comfort the family. She felt that her presence was valuable and comforting to the family while they were going through such a difficult time. Little did she know that according to their religious belief the position in which she was standing was actually hindering the patient's dying process.

I would have said they were maybe Middle East or Middle Europe, something like that. Anyway, I was standing at the end of the bed and nothing much was going on but trying to be supportive and quiet and calm. And I could realize that people weren't happy and I didn't quite know what was wrong so I'm looking around and finally somebody said to me [inaudible - pos 18.02], so it's just trying to be sensitive cause standing at the end of the bed was important because the spirit leaves out through the bottom of the body, sort of out through the feet, and I was in the way. (FCC-07; p. 7).

All of the participants shared that their primary goal was to care for the patientfamily and make their cancer journey as comfortable as possible. When the participants did not have an understanding or knowledge of the patient-family's specific culture, they observed that they were unable to adequately provide FCC. 
Fear of Precipitating a Negative Event or Outcome when Lacking Cultural

\section{Knowledge}

As a result of not fully knowing and understanding the patient-family's culture, the participants expressed that sometimes they unintentionally made comments or said something that undermined the nurse patient-family relationship. Such comments and statements had the potential to be offensive and even worsen an already difficult situation.

And if you don't know what their culture is and how they interact as a family you may say that - I may say things that would be appropriate in our culture but it wouldn't be for them... So I may think I'm giving family centered care because I'm explaining something to them but really I've done the opposite and I've made things bad for them as a family because I haven't worked in kind of what their framework is for what is supposed to happen in a family. (FCC-20; p5).

I would never say that I've had a trouble with giving care here kind of on a moral or an ethical dilemma; it's more just that I don't fully understand their culture sometimes and so I will have done or said something that in western culture is acceptable to talk about cancer or talk about a tumor or a mass and I said something that for the family was inappropriate. And then very much, completely unknowingly, you know I certainly would never have done that if I had known that that wasn't appropriate for them, but yeah - and you step back and you have big regret. Oh my gosh, like what kind of implications is this going to have now for this family. (FCC20; p9-10).

\section{Language Barriers}

Participants noted that it was important to inform parents about the nursing care provided to their children. At times, the participants identified that they were unable to communicate and educate parents and family members because of a language barrier.

Well, we certainly have lots of families, immigrant families, now and sometimes the biggest challenge isn't necessarily their cultural beliefs but the fact that they don't speak English, and it's really hard to provide family centered care to a family that doesn't understand you. You come to learn how much you rely on language and that form of verbal communication to provide the care that you do. (FCC-09; p.10).

One nurse shared an experience of caring for a patient whose parents were deaf.

She was unable to fully communicate with the parents because of their hearing 
impairment. This nurse expressed that she did not know if the parents fully understood the care she was providing to their child and was unable to fully inform them about the condition of their child. This also limited and hindered the parents' ability to parent and participate in the care of their child.

I did have a patient whose parents were both deaf and so sometimes - they had a special piece of equipment where they could type in things if it was hooked up to a phone or something. I can't remember, it was years ago. So I always sometimes felt like, do they know what I'm really doing? But in saying that I think they were really smart and they were really good at reading lips. But I just never - I just was kind of worried, like have I missed something and they're not - yeah. (FCC-14; p.6).

\section{Non-Traditional Family}

Participants observed that they often cared for patients with parents who had 'family-issues,' e.g., they were divorced. In many of these situations, parents did not get along and there was tension between them when they were together with their child. Participants observed that the care they provided to such families was segregated, because they were unable to speak to both parents at the same time about their child's care. As a result, different information was shared with each parent and there was the possibility that both parents did not have complete understanding of their child's disease or treatment.

And also when there are family issues in families like divorced parents or - then it's very segregated care with the parents so one parent will know more than the other. (FCC-12; p.1).

When the parents were separated and could not be in the same room, the nurse had to educate and provide information on two different occasions. This was time consuming and meant that each parent may not have received the same quality of information regarding their child's situation. 
And if you get a set of parents who have gone through an awful separation or awful divorce and they can't be in the same room at the same time, how do you give family centered care in a timely way because you have to do the exact same thing twice and you have to go through all the information with one parent and then all the information with the other. And when you do it twice do you jip one parent perhaps because they're now the second parent to be seen and you're more conscientious of time? Do you go through the information in a shorter fashion, do you now have as much patients with them for questions? Do you shortcut and skip some things? It's possible. You try not to but it could be possible cause you may not know, oh it wasn't with this parent that I discussed it; it was with the other parent. But in your mind you had discussed it with the parent in from of you. So just the fact that the families aren't intact anymore, that is a big barrier to family centered care too. (FCC-20; p.5).

Occasionally, when parents underwent a divorce or separation the child was caught in a legal war. In situations like this, step parents became involved in the care, and often did not have legal custody or authority to offer consent for treatments. This placed additional stress and work on the participants. He or she had to understand the legal implications regarding the step parents, custodial and non-custodial parents, and shared custody parental arrangements. A participant shared such an experience below:

And then the other too is just with the traditional definition of family changing now there's always extended family like step-parents. There are lots of divorces; there are lots of step-parents. You don't know what the legality behind it is sometimes when you get parents who play off each other to say, "I'm the only one entitled to this information. I have legal recourse if you give information to the other parent." And you have to do a lot of background work to know if that's really true or not. So you can get - yeah, you can't give full family centered care until you know what they're at for legal pieces who you can give and can't give information to and who the caregivers are in the family. (FCC-20; p.5).

\section{Contradictions and Tensions between Nurses and Families}

While providing care for the patients and their families, participants identified that there were situations in which they did not agree with the decisions that the families made on behalf of the patients. There were also times when the participants did not agree with the families' beliefs or were unable to honor a request that families had made. 


\section{Disagreeing with Decisions Made by Patient-Families}

The participants shared that it was difficult to provide FCC when they did not agree with the patient-family's decision regarding treatment plans.

We had one little girl a while ago I guess now, but anyways when she was diagnosed her family decided - she was 10 and she was very, very smart - her family decided they didn't want to tell her that she had cancer. And so we weren't allowed anywhere around the child or around the room to use the cancer word. She got chemo and knew she was getting chemo, and her hair fell out, and she was sick, and there was all these issues and, you know, we just weren't allowed to talk about it and you went along. And they just told her that her liver was sick and she needed to get medicine to help her. And I mean, that's their choice and that's their child and I'm not in that situation but it just felt like, I mean, she's - it's not like she's two years old. I mean, she's 10 years old and she knows something's wrong. It just felt like a huge conflict of interest to go in there and, you know, we try or at least I try very hard to, you know, talk to people and figure out what they're going through because, I mean, it is an uncomfortable topic a lot of the times, you know, to find out how somebody feels about having cancer. But I think it makes a huge difference then to have to tiptoe around the subject and pretend like everything's good, she's not really sick, just needs a little bit of medicine. It was really, really hard. And then I found it even harder as nursing staff to not judge them for that decision because, like, personally that is just not something that I would ever do. Like, that's just not a decision that I would make. But that's their decision and it was super hard. I think we - a lot of people got into a habit of, you know, thinking maybe they're bad parents or something because they're making this decision that seems a little bit crazy to us. So that was really hard. (FCC$19 ;$ p. 6-7).

Within pediatric oncology nursing the patient-family is a part of the treatment plan decision making. This can negatively impact the nurse, as well as the family, if the nurse does not agree with the decision that was made.

In the following example a nurse explored a situation in which she cared for a patient who had cancer of the bone in one of her legs. In order for the physicians to be confident the cancer would not re-occur, they strongly suggested that this patient have her leg amputated. This culturally diverse family highly valued female beauty as a way to 
become eligible for marriage. As a result, the patient-family decided not to have the leg amputated in order that she would be eligible for marriage when she got older.

An East Indian girl who has come through clinic now and had cancer of her leg, in order to make her into kind of a more cured position they would like to amputate her leg and in Indian culture if a female has any of their body kind of manipulated it doesn't make them as profitable for marriage. So I struggle with thinking if we don't amputate her leg she may not live to get to a marrying age, so why think about how profitable she'll be for marriage if she won't even be alive for marriage?.. so yeah, I mean there's always little struggles but it's hard to provide care for that family when you're limited in what you can say in front of their child and in speaking with them and you put out all of the information to try and give them their knowledge of power - they come at their choice from a different angle. And do I have the right to say to them, "have you considered that if she doesn't have a leg amputation you may not get to marriage?" You don't know if that's acceptable in their culture either because things can be very, very hush-hush. (FCC-21; p. 10).

As demonstrated above, parents and family can make care decisions that the nurses know could harm the patient. This nurse was not able to voice what she believed, but had to be silent and continue to provide care for the patient-family. As a result, the participant felt that she was unable to fully educate the patient and family regarding their treatment outcomes, which also made her uncomfortable. The participant did not feel that she was helping the patient-family, because in such instance there was a treatment option that had much better outcomes.

Participants shared instances where the parents tried to protect their child by not telling her/him about a cancer diagnosis or that the care had transitioned to palliative. Such situations were morally confusing and distressing for the participants because they did not agree with the parent's decision to withhold information from the child. This placed the participants in a difficult position because they conducted procedures on these patients and gave them chemotherapy all the while the patients did not understand what was happening. FCC involves keeping the patient-family fully informed. When the 
participants were unable to tell the child that she/he had cancer or was palliative, they entered into a moral and ethical dilemma. As a consequence, participants stated that they were unable to fully provide FCC.

Morally, I am like, come on, your kid knows, he is 13 years old, he knows he is passing. Can we just talk about it, it's just this huge elephant in the room, he even knows what the palliative suite is and he's in there. Can we just get rid of the elephant, do you know what I mean, that is so hard. (FCC-06; p.13).

Another barrier to FCC described during the interviews was when the parents did not accept that their child was dying and insisted on continuing with various treatment regimens.

I mean, situations where a family just can't let us stop or won't let us stop, and I don't know that that's culture necessarily but it's sure hard to understand for us when we know that it's time to just allow the child to die. (FCC-07; p.8).

The nurses who cared for these children knew from experience that they were going to die, but the parents were not willing to give up on treatment. Parents pushed to have their children receive treatments and encouraged their children to keep fighting to live. This was difficult for the nurses caring for these children because they saw the children suffering from the side effects of chemotherapy rather than peacefully passing away.

One participant described a situation where a mother her child to be discharged from the hospital against the physicians' recommendations. The mother strongly believed that she could manage at home and provide the same care that her child would receive in the hospital. This child had very low hematology counts which put her at a high risk of contracting an infection. Therefore, the medical team disagreed with this mother's belief that she could care for her child at home. 
The part where I think it was difficult was because a lot of us didn't necessarily agree with some of the mom's beliefs and what she wanted, and so us as a team not believing what she believes. I think we made it difficult for her and we weren't able to accommodate a lot of things, although we were able to accommodate some things. (FCC-02; p.2).

When nurses practice within the FCC framework, they do what they can to make the parents feel comfortable and involved in decision making. In this situation the opposite occurred, which had a negative impact on the mother and prevented FCC from being practiced.

\section{Disagreement with Families' Spiritual and Religious Beliefs}

Participants noted that a barrier to providing FCC was the spiritual and religious beliefs of the patient-family. More specifically, some participants felt that their personal beliefs clashed and conflicted with those of the patient-family. More specifically, some participants struggled with the Jehovah Witness religion.

Jehovah Witness was identified as a specific religion that was a barrier to FCC among pediatric oncology because of their belief against receiving blood products. When a child is diagnosed with cance they receive high doses of chemotherapy that drastically decrease their hematology blood counts. In order to literally keep these children alive, they must receive multiple blood product transfusions to increase their hematology blood counts.

One instance was described by multiple participants where a teenage girl within the Jehovah Witness faith refused to receive blood products. As a result, this case went to the courts and she was taken under the custody of the state (province). The medical team administered blood products to this patient and had to restrain her hands and feet when 
doing so. Many of the nurses greatly struggled with having to force treatments upon the

patient.

But we did have a patient who was on the unit of a different faith background from the Jehovah Witness background, and the struggle was in place both by what we had to do for her because we did give her blood products against the family's will. And I was never present to do that because it happened on the unit and I'm here in the clinic, but I did hear of the nurses who struggled with the actual pieces of I mean physically restraining her to give her a blood product, you know, having her actually - they actually did restrain her wrists and her ankles to the bed so that there wasn't - she was 16 and she could be very, very strong. So to avoid her being able to move they actually did restrain her. I mean, this is all third-hand information; I didn't see it, I didn't experience it but I do know that that has happened and I struggled with that just hearing about it. To know that there was a family who did not support blood products, here was a 16-year-old child who did not support blood products and so from both perspectives they didn't want something to be given and we gave it. And the medical part of me said if she doesn't get this blood product she could die. She's got low platelets or she'd got low hemoglobin. I mean, this could threaten her life.

And to me, because I don't have an adversity for blood products, it seems so wasteful for lack of a better word, that someone would put their own life in jeopardy because of that, something that to me seemed so solvable. If you have a blood product you won't have your life in jeopardy. But I don't have their faith background. So, you know, I have to step out of it and say that's not my life that I'm making a decision on. It's their life and they have the right to make the decision about their life, not me. So I would really have struggled if I had to be a part of that to walk in the room and hang a blood product on someone who believes that part of your soul resides in your blood. (FCC-20; p.9).

Part of the struggle that the participants had in caring for this patient was not being able to provide adequate care. This child was suffering and not receiving life saving treatment because of her and her family's religious belief. And when treatment was provided, the child was physically restrained and the full weight of the law forced the blood products upon her. Obviously, these nurses had a difficult time establishing a relationship with the patient-family. Therefore, they felt that they were unable to provide FCC in this situation.

It was hard because you knew there was a standard of care that we could provide and yet there was a barrier to it. (FCC-14; p.6). 
Some participants shared instances when they had to care for patient-families with the same faith, but they had deviations of beliefs within that faith. This occurred particularly within the Christian faith. This was difficult for the nurses who practiced the Christian faith because it challenged their own personal Christian beliefs.

I had a really difficult time with one of our patients who passed away. His family was really, as far as the faith perspective, really what I felt gave God limitation. Like, we're just only praying for that miracle for their son to be healed, which is totally possible in what I believe, but it was like there was no other option. And I had a - and I cared for them a lot in the time before he died and I just - it was a huge struggle for me because I was just so worried that they were going to completely lose their faith and be completely shattered when he did die and that miracle didn't happen. So in all of that my way of caring form them was just to be there and be supportive and for them to know that I was praying and that did have hope to you know, because I didn't want that hope to be crushed. (FCC-17; p.5).

Participants who had different religious beliefs than the patient-family also struggled in providing FCC. The following participant explained how she struggled when the mother and father had very different beliefs than her own.

But it did clash with my beliefs and it makes you uncomfortable inside. (FCC-02; p.10).

This particular nurse struggled with the fact that the parents had strong beliefs that did not allow the family to understand or realize that their child was dying. It was challenging for this nurse because she knew from experience that the results of this child's illness was not going to be what the parents hoped for. This type of situation caused participants to struggle because they could not fully and openly discuss the parents' beliefs as they felt uncomfortable doing so.

One participant shared how she found it difficult to discuss things with parents who had extremely different "cancer causation" beliefs than hers. She suggested that the parents' beliefs were a barrier to providing FCC. 
Uh, we had one kid who died just before Christmas and their family was super religious. Like, really, really, really over the top fundamentalist Christian and they believed that he got cancer because they sinned. And they believe that the reason he was dying was it was their fault and it was God punishing them for doing bad things or whatever. It was very - I did not believe what they were saying cause the science part of me is like, no, he got cancer because of this. It's not anything that you did and it's - you could tell them that but really what is the point? They don't - that's not my place to tell them, "Actually no, this is why he got cancer." It was just really hard because every time you go into the room and he'd be getting sick they would be like you could just tell they were feeling so guilty and it made me so sad that that's the way they were coping with it. Like, it was so not their fault at all and it just - I don't know, it just made me really sad. But there wasn't really anything we could say or do for them kind of thing. That's just the way that they were coping with it. (FCC$13 ;$ p.9)

\section{Unable to Fulfill Parents' Requests and Wishes}

Often times, pediatric oncology nurses must conduct procedures that go against the parents' wishes. This can be very difficult for the nurses because they know that going against the wishes of the parents can negatively impact them. It was also frustrating for the participants because they knew that the parents may not have been medically informed and simply did not understand the rationale for conducting such procedures.

I think the biggest thing for me is I can't always do what they ask me to do because either medically it would be unsafe, legally it's not in my scope, or there's somebody who's better able to do what they're asking to be done, whether it's a different discipline or a different nurse whose got a different skill set. (FCC-20; p.7).

One participant described in great detail how she had to admit a patient against the parent's wishes because he [son] had a severe allergic reaction to a blood product and admission was not optional. There was simply no negotiating with the parent the decision to admit the patient because this child's well-being was at stake. Part of FCC is being able to meet the parent's requests and involve them in care decisions. In this situation, this participant had to dictate the care plan to the parent. This made her feel distant and cold, which prevented her from providing FCC. 
We had to admit him and I - there was no negotiating on that and she [mother] just didn't want - I think she - well, she felt that she could handle him and all that but it wasn't - and it was done with the oncologist and all that - but I tended to be the one who bore the brunt of her anger, and I understood that. It's not that it doesn't kind of affect you at the time; it did affect me very much because I tend to work very well with this family and such but it happens. And I knew I could presume somewhat that it was partly her managing things and something unexpected was very hard for her to sort of turn around for family commitments and all that. (FCC-04; p.10).

Parents of children with cancer are under great amounts of stress, and are searching and desperate to do anything that will keep their child alive and comfortable.

Therefore, it was difficult for the participants when they must say "no" to something that parents ask because they know that it makes the family feel alone and helpless.

\section{Lack of Time and Increasing Acuity of Patients}

The unit and clinic in which the participants worked were very busy and demanding. Patients within this specialty had complex oncology diseases and were acutely ill in comparison to other pediatric settings. Barriers to FCC that were identified by every participant involved in this study included: not enough time, high acuity of patients, and staffing.

\section{Time}

This specialty involves many different treatments and procedures that must be completed within time constraints. Therefore, the participants noted their practice was often tasked focused.

As I say, we can get very focused I think sometimes on the tasks. It's a very technically challenging setting so I don't know that we can always have the time that we should to do - yeah, to focus in that way. (FCC-07; p.3).

I think in our setting particularly we spend a lot of time on the technical pieces of care right now is the sense I have. (FCC-07; p.3-4).

Given the care demands associated with these tasks and interventions, the participants noted that they did not have much time to focus on the psychosocial or 
educational needs of the patient-family. All of the participants in this study stated that lack of time was a barrier to providing FCC.

Boy. I think barriers and challenges is always not enough time in the day. We're a busy unit. Transplant is increasing so patient load and acuity - everyone's running around trying to meet the basic needs, and so often times you don't have the time to sit with a family and just listen to what they have to say or to spend the time like you'd like to. (FCC-01; p.3).

Participants concluded that they did not provide FCC when they were unable to take the time to talk with the families. The following textual excerpts illustrate and support this observation:

Just if it would be really busy and under-staffed then you can't. You just have to make up your schedule for the day and you don't have time to talk to families, so you're just providing the basic nursing care. Yeah, I don't feel like that's family centered. (FCC-03; p.9).

And then just one other thing too, sorry, is also just not having the time to be able to really be present sometimes with families.... And it's like there's eight other patients out there and you're expected just to go, go, go, go, go and a physical exam should be no more than five minutes, and get in and do your vitals and your height and your weight and your meds and get out. So that can be really challenging too. (FCC-20; p.4).

Participants identified that an important part of FCC was taking time to educate the patient-family about the diagnosis and treatment. When nurses were too busy to do so, they felt that they were not providing FCC.

On busy days totally, because you're throwing in this chemo and the parents are like, "Wait, what is that?" And you're like, "Shoot! I didn't even tell you what it is," and half the time you're giving it and you know what it is but in your own words, so like - and you've really got to I guess dumb things up for parents. And I don't mean that in a bad way but not medical jargon obviously. So, yeah.” (FCC-12; p.1).

It took time to create relationships with the patient-family. When participants were task focused and had limited time, they were unable to create therapeutic relationships with the patient-family. The following participant expressed this concern: 
I think how busy things get sometimes, cause that's one of my favorite parts of the job is getting to build those relationships with the families and stuff like that, and so I think, yeah, when things are really, really busy you don't have time to do that as much. You just have to get in and get your tasks done and leave. And so I'd say that would be a definite barrier is just how busy things are. Yeah, I guess that's kind of the main one. (FCC-17; p.2).

Another element of FCC was taking time to play with the child and make him or her feel safe and comfortable with the medical staff. When the nurses were pressed for time, they were unable to foster therapeutic relationships with the children.

Yeah, I hate when it's just rush and say, "Hey." I really don't like when - yeah, when you're so focused on all the things that need to get done to or for the patient. You kind of miss out on a bit of the connecting with their parents and - yeah, when it's so task focused it's so - for myself I find it really hard cause I love to just sit down and chat or have a bit more - even just play time with the child so that there's that part of their therapy too, not just the medications and tasks that need to be done. Like, I think a lot of the play and fun is really beneficial to their getting better too. (FCC-18; p.4).

This example revealed the positive outcome that can occur if nurses have time to spend with the patient-family. It was obvious during the interviews that each of the participants knew what type of care they wanted to provide for patient-families, but time constraints undermined this desired and ideal nursing care.

One nurse shared an experience from when she was a student that demonstrated the possibilities of what pediatric oncology nurses can accomplish with families, time permitting. As a student, she had extra time to spend with a particular family. She expressed that she went and sat with the parents during her spare time. Upon doing so, they opened up with her because she was present and gave them the time to express their concerns and feelings.

I mean, I woke up their child when she was asleep and she was having trouble sleeping. And just a few of their, I guess, things that had bothered them. And I just had time because I was a student and I was just looking for something to do so that 
was just something to do. It wasn't anyone else's fault or not; everyone was really busy, this was a really busy time on the unit. It was quite under-staffed at that time. But I just had the time and I don't think anyone had - I don't think this family had had a chance to say a few of these things. And then just listening to them and a few of their concerns, a few of them we could change. Like, we could do the blood work at 6:00 in the morning and just - I can't remember what the other things were but we could negotiate a little bit. And just doing that for them, that just gave them some control. Like, "Wow, this isn't maybe as horrible as it needs to be." We can actually change a few of the things that they found really difficult. (FCC-03; p.10).

\section{Patient Acuity}

Participants revealed that they provided care to many acutely ill patients. These patients required complex treatments, frequent monitoring and atypical procedures. This was stressful for the participants because increased patient acuity translated into increased time spent with such patients. Also, the more acutely ill the patients were, the more task focused the participants became as a means to keep the patients alive.

Therefore, patient acuity was described as a barrier to FCC.

I think time constraints sometimes can do it based on the acuity and our bed census. And that's not intentional but I think it's just there's such a focus sometimes on what you need to get done as far as tasks go. (FCC-14; p.3).

Interviewer: Can you describe an experience where you were unable to provide family centered care?

Participant: Uh, I guess probably in those moments when a patient's acutely ill. Thinking back, you know, you're so task orientated or focused and maybe not providing enough information of what was going on at that point in time. (FCC-14; p.1).

When the nurses had a patient who was more acutely ill than their other patients, the majority of their time was spent with the acutely ill patient. As a result, their other patients suffered because they did not have the time to provide FCC.

I think sometimes even the acuity of the patient can definitely be a barrier, or the patient load itself. Like, if you've got one really sick kid and two that are okay sometimes it's hard cause you're spending most of your time with the sickest child 
and then you're kind of missing out on a bit of the involvement with the other kids. You're kind of just running in and out doing tasks other than, yeah, focusing on really therapeutic relationships with all of them because you're so busy in one certain part. I think that's definitely the - if the patient load was lighter I think it's easier to provide more holistic care. But at the moment I don't think there's a lot they can do about that just with how many nurses they can get to work. But I would say that's probably the biggest barrier. Or if there's just a lot to do task-wise it's hard to fit that all in, plus then there's often the guilty feelings on top of that so it just kind of wrecks your day. (FCC-18; p.4).

\section{Inadequate Staffing}

Given such patient acuity, an obvious solution would entail an increase in the staff compliment. This was usually not feasible because of the current nursing staff shortage and budget constraints that Alberta Health Services is experiencing. Therefore, a barrier to FCC that the participants pointed out was not having time to provide FCC to their patients because they had a heavy case load due to being short staffed.

Also, the participants indicated that a barrier to FCC was staffing issues such as working without the required baseline number of nurses. Staffing issues caused the participants to take extra patients in their case load, which meant that they had less time to spend with each patient-family. During such times, the participants had to focus on getting their tasks done because patient safety came first. As a result, they were unable to incorporate the family into the care and provide FCC. The following quotes illustrate this finding:

Maybe resources in terms of staff, like, if there were more people taking care of the patients. You know, your patient load sometimes dictates how busy you are, so if you weren't looking after five or six kids then you might have more time. So that would be something that prevents me. Uh, that's probably the biggest one that I can think of. (FCC-01; p.4).

Just if it would be really busy and under-staffed then you can't. You just have to make up your schedule for the day and you don't have time to talk to families, so 
you're just providing the basic nursing care. Yeah, I don't feel like that's family centered. (FCC-03; p.9).

New Nurse

Participants identified that a challenge to providing FCC was being a new nurse. Pediatric oncology nursing is a complex specialty that requires nurses to be skilled and knowledgeable of intricate nursing tasks. When the nurse is comfortable conducting such tasks, she/he is able to communicate and provide FCC while conducting the tasks: ideally the tasks become a means to interact with the family and provide FCC.

Currently, a majority of the nursing staff on the inpatient unit are relatively new nurses. The participants expressed that when nurses are new to this specialty they do not have the specific skills and knowledge needed to provide FCC because these are acquired over time with experience. As a result, the new nurses were not able to provide FCC while conducting tasks because they were too focused on performing the tasks properly and safely.

I think sometimes being a new nurse I still get worried a little bit about the tasks cause they're new and they're sometimes more difficult here. (FCC-18; p.5).

The participants noted that part of being able to provide FCC was having the ability to look at the big picture and prioritize. It was noted that new nurses' focus was on learning how to perform the tasks in a timely and safe manner. The new nurses struggled with determining how much time to spend being present and communicating with the patient-family and yet have adequate time to accomplish their nursing tasks.

And I think balancing that, tasks with family centered care, becomes easier the more experience you have and the longer that you've been a nurse with pediatric oncology. And I don't want to say that it's a negotiation but I think an experienced nurse is just better able to judge how best to handle what needs absolutely must need to get done with what can wait and what's best for the patient. (FCC-14; p.3). 
Yeah, I think so. I think it's hard because there are some families that you could just talk to for a whole day kind of thing, and you go in the room and they just talk, talk, talk, talk, talk and it's bad because there's so much other stuff that I'm thinking about doing. I'm talking to them but then I'm actually thinking, oh, I need to go give this med and I need to get this med and I need to go get this person's weight and do all of this. I don't feel like sometimes I'm completely engaging in the conversation just because I'm like, “Okay, yeah. I know, I know. I know." and then it's like, "Okay, see you later." And like, I could see maybe some parents thinking that man, that ****, she's not really interested in talking to us because I'm like, thinking about 100 other things while I'm still talking to them at the same time. (FCC-13; p.3).

As the new nurses became more confident and comfortable with the tasks, they

were then able to provide FCC while performing the tasks.

Interviewer: So in a way when nurses get more comfortable and confident in tasks they're able to incorporate family centered care a bit better or -

Participant: I think so and I think also some of it is just confidence level in their own abilities and what they're comfortable doing and not doing. (FCC-14; p.3).

Yeah, absolutely. And I think with me I found that now that I've been here for a little while and I'm more comfortable with the medical side of things and the skills and I know that I have the ability to get it done in a day and I know what to do, I'm able to focus more on the other stuff: the family centered care, the psychosocial, all that other stuff. But when you're new there's so much information and the learning curve is so steep, and it's intimidating because these families know so much that you just want to get in and do it properly and, you know, not embarrass yourself or not know the answer. And so, yeah, you don't even - I wouldn't have even my first six months considered anything besides come in, here's the medical picture, do this, give the drugs, and you just - you can't. It's too overwhelming. There's too much to learn. (FCC-19; p.5).

\section{Budget and Resources}

The participants stated that there was an increase in number of transplants

occurring and that the program was increasing in size. Participants alluded to the fact that even though the program was growing and there was an increase in number of transplants performed, no increase in nursing staff coincided with this.

But then on the other side of it we're a growing program and our numbers are just climbing and I don't think that we have all of the resources that we need to accommodate the increasing numbers. (FCC-03; p.3). 
Participants indicated that they experienced high levels of stress and were busier than before in light of the expanding program without hiring more nursing staff. This was related to the department budget and was observed as a barrier to providing FCC.

So I think resources, probably financially, finances. (FCC-01; p.3).

One nurse shared that as a result of the growing program without increased staffing necessitated the creation of a new policy to accommodate the large numbers of patients. This policy insisted that the patient-family came in the day before their clinic date to have blood work done. This was put in place to decrease wait time for blood results, and thus move more patients through the clinic faster. Such policies upset some of the families and prevented nurses from providing FCC.

We changed our policy probably just when I started, so that would have been four months ago or five months ago when I started my primary nurse role where we're asking kids to get their blood work done the day before chemotherapy to see if their counts are ready. And most families, they understood why and it was fine. One family refused and I couldn't just listen to their concerns and say, 'Well those are good reasons why you're not going to,' because it was really management driven and so I had to have a meeting with management and the family and be really forceful with that. So that was a situation where we just dictated. And if there's no room for flexibility because management has maybe dictated that, then you just have to say, 'This is the policy and I'm just following the policy.' (FCC-03; p.11).

\section{Limited Space and Lack of Resources}

The participants expressed the limited space and lack of resources for the patientfamilies while in the hospital constituted a barrier to FCC. They stated that they needed adequate space to perform their tasks and concentrate on the patients. At times, they had to limit the number of friends and family members at the bedside because of safety concerns. One nurse expressed how she felt that limiting the number of friends and family members at the bedside was a barrier to FCC. 
Uh, you know, and it goes the opposite way too when - so we work in these small conditions, right, and there's ways to try and maximize it the most we can but there's also - it's just sheer physical space; we can't accommodate everybody here unfortunately. If we say, 'Hey, come on in with everyone,' we wouldn't have the space around the stretchers to do the work that we need to do. We wouldn't be able to do it safely because we need noise to be somewhat at a quiet roar instead of a circle chaos, which it normally is with kids. But, you know, we're doing chemotherapy drugs, we're doing procedures, we're giving blood products, we're monitoring [inaudible - pos 8.10]. Like, you just have to have all of your ears and your eyes open at all times and you have to have that intuition that's at its peak. And if you have every physical space filled with every family member you run out of space and you run out of that ability to then be safe. So in that way it's hard to work for family centered care because you want the family to be together through such a long process, like I mean, these kids could be six months for treatment, it could be three years for treatment so you want them to experience that all together if that's what they choose, but sometimes you have to put a limit on how many people can come. And that's really hard because then I don't feel like we give family centered care. (FCC-20; p.3)

The participants expressed that part of FCC was enabling the family to be together during the patient's cancer journey. When they had to limit the number of family members and friends present they were unable to fully practice FCC.

The patient-family is confined to the room during their hospital stay, and to a cot while in the clinic. Participants described situations when they were unable to help the patient-family be comfortable and content while in the hospital because the patientfamily was expected to stay in the room or seated around the cot. There were limited activities that the patient-family could do since they did not have the choice to move around. The following nurses clearly spoke to this issue:

So our families are.. honestly, when our families are here where are they are going to go? They don't have anywhere. A lot of them had said I wish they would have thought of maybe putting in like a gym even for kids to be active, they want to be. A lot of teams want to be. A lot of times I just get sick of them sitting in bed watching TV because they are sick of it too. So I think that way I think the hospital could have. I just feel like we are so excluded from that whole zone, you know removed from everyone. (FCC-06; p.7). 
I mean they try to have movie night on the unit, but it is just for them to get out to society, you know.. and they can't even to go the cafeteria. They have to pretty much have to go from our unit off. There is just things like that, that I think the children's hospital, they claim to be so family centered, but they have really excluded the immunosuppressed kids. (FCC-06; p.7).

\section{Lack of Education about FCC for Nurses and Health Care Providers}

The participants expressed that a barrier to providing FCC was a lack of understanding about the philosophy itself. They would hear the word mentioned in their workplace and knew that the $\mathrm{ACH}$ was a family-centered facility, but the majority of the participants never received any formal education or training about FCC. They stated that it was difficult for them to implement FCC into their practice when they did not have an understanding of the philosophy itself.

People don't get a good understanding of what family centered care is, you know, maybe we don't get enough opportunities to learn about it in a theoretical sense. (FCC-02; p.3).

Participants indicated that learning about FCC within this program was not a priority because it is science focused and research based. Theories and philosophies that are not science based were not as readily embraced. Consequently, the participants noted that there was a lack of understanding among the medical staff on how to implement FCC into their practice and the positive implications of such philosophy regarding care.

I think a lot of people don't understand family centered care. They just think it's a fluffy word. You say something with a drug in it and everyone is involved. It's just that medical knowledge sometimes, but I think people think it is all fluffy but I don't think then they really understand it, they might practice it, but they are unwilling to kind of put a label on it because they just think it is... do you know what I mean? (FCC-06; p.8).

Although there is research and theory development around FCC, only three of the participants shared or expressed that they had read or been exposed to such literature. There was no formal education or training opportunities for nursing staff to learn about 
FCC at the ACH except for a half an hour presentation they received during their staff orientation.

Yeah. I don't think that they $(\mathrm{ACH})$ really show it in a way. Like, I think we talked about it in the general nursing orientation but I can't even remember. So I'm sure they support it but there's not - like I said, no requirement for education so there's obviously not enough funding for it for us nurses to dive right into it. (FCC-12; p.2).

One participant expressed her frustrations concerning how she was expected to understand FCC and implement it into her practice without receiving formal education or training:

Uh, education, like on our part as the nurses. Like, you kind of get thrown into it without really knowing what it is. Likes, it's not a requirement to take a course. And also there's family issues in families like... but more just education and just not knowing even what it is like. (FCC-12; p.1).

Every participant recognized that FCC was an important and valuable part of their practice. A majority of the participants, however, expressed that they lacked education and training on the philosophy of FCC and stated that this was an area that needed improvement. Over $80 \%$ of participants stated that they would appreciate more education and training on the philosophy of FCC.

So I think I've had a few opportunities like that, but certainly there's a lot of room for more. (FCC-02; p.4).

\section{Difficult Working Relationships}

Participants indicated that certain attributes and characteristics of the patientfamily working relationship was a barrier to providing FCC. These included: non trusting families and difficult families.

\section{Non Trusting Families}

Participants identified that a barrier to FCC was when the patient-family did not trust the medical staff. This type of patient-family frequently questioned what the nurse 
or physician did, which made it difficult for the participants to provide FCC because they always felt on edge.

And not every family is easy to work with, we know that, but I try to put those things aside because we all get tired and we all sort of - it is harder to deal with families that are more confrontational or less trusting. I always find it difficult in a way of saying I guess difficult in the way I always find it - it disappoints me when a family doesn't have trust in the team and I try to work at little pieces of that because we all come with our different experiences and that's going to happen. (FCC-04; p.3).

It was discouraging for the participants when the family did not trust the medical staff because they were unable to get all parties (i.e., patient-family and members of the health care team) on the same page. The participants found that they clashed and experienced conflicts with the non trusting patient-family.

Patient-family's who did not trust the nursing staff were confrontational. This was stressful for the nursing staff because they had to use their time and energy to work out issues with the family rather than focus on caring for the patient.

I think was before your time but a very challenging situation of a little girl who failed three transplants and having her fourth was when she actually died with those complications. But mom was very challenging, very much in your face physically. Like, we'd be standing and - so you were trying very hard to work with the child. There was a grandmother, the mother and father, and a sibling and a baby, which doesn't count, but a sister that was a little bit older. And you're trying so hard. And I must say, at times I felt very discouraged in that situation cause I just couldn't seem to bring us always to the table together. I didn't start with them so you come into situations that have developed and progressed but it's - still you would hope that you could help to bring things to a better level that as much as we tried to even institute a primary core it was just a challenge. So I do feel discouraged there that it was hard to get us all feeling like we're playing as a part of the team. You always felt like you were on the defensive and trying to fix and to - you never got to a point where you were working together or anticipating. It was just always this responsive, defensive kind of - so that - disappointing probably, that's probably a good example of a disappointing situation. Yeah. (FCC-07; p.2).

When the patient-family did not trust the nursing staff they never truly became part of the team. A collaborative working relationship was not created, and therefore, FCC was not fully incorporated into nursing practice in such situations. 


\section{Difficult Families}

A majority of the participants mentioned that a barrier to providing FCC was 'difficult families.' These were families that the participants found to be difficult to please and provide care for. This was usually because of the personalities of the family, since they were not cooperative or flexible. Such families made it difficult for the nurse to provide FCC and moreover, the health care team often distanced themselves from such difficult care situations.

But I think there were a lot of things that because we didn't agree with her or necessarily even enjoy her company that much, you know, she was very difficult to deal with, I think we almost united as a team almost against her in a way. So I think that was an example of maybe not good family centered. (FCC-02; p.2).

Families who were difficult to work with were not a priority because they required more time on the nurses part when interacting with them. As a result, difficult families received less time with the medical staff and limited FCC.

I think sometimes other times we, depending on a family and maybe the relationship we have with them, we'll call them sooner than others cause - and this is probably really terrible and wrong but if a family is a difficult family to deal with then you'll wait to make that phone call. That's probably wrong [inaudible - pos 15.50] happens." (FCC-03; p.4).

Difficult families made frequent unreasonable requests of the nursing staff. This was overwhelming for nurses because they were already pressed for time while caring for multiple and acutely ill patients.

For example, I guess there's one family that when - they came here from Edmonton and they were here - they've been here forever, and when they first came it was like they didn't like us, they couldn't get the hang of us and they just didn't know how to deal with us, and I think we just didn't know how to deal with them and they just have really specific needs and really specific requests. (FCC-13; p.4). 


\section{Brave Face}

The participants stated that they knew the patient's test results before the family was informed by their respective physician. This was very difficult for the participants because sometimes the test results showed that the patient had relapsed and the participants were not able to inform the family. The family was aware that blood work was done, thus, they constantly asked the nurses for the results. However, the nurses had to lie or avoid the situation and put on a "brave face" when they knew the results were "bad". They had to act "happy" or in a normal fashion as a means to not let the parents assume that the test results were "bad". This was a struggle for the participants.

I think it is awkward for nursing sometimes because we do know the results of the bone marrow before a family, before the physician might have time to present it to them, so that's hard. Or you might know the result of a CT or an MRI before the family does. If it's good news fine, you don't usually have that internal struggle but if it's bad news it's hard, cause there could be a day or two that goes by on the unit when you know that there's blast in the marrow or something and the family isn't aware yet cause we're waiting for the - slow and stuff. And that's hard. It's an awkward position to be in and I think a lot of nurses struggle with that. Like, how to carry on that brave face that you don't know when truly you do. (FCC-14; p.7).

When this happened, the participants stated that they still had to go into the room with a smile on their face and pretend as if nothing was wrong. The participants knew that when the patient-family found out the results they were going to be devastated. The participants stated that this was a barrier to FCC because they had to lie and not be authentic in relationship with the patient-family; they were not able to be truthful and honest in the moment.

For example we'll get their blood work in the morning and it'll show that they've relapsed and we still have to go and nurse until the doctors go in there and tell them and you know that the outcome's going to be horrible and that this one day is going to change their life forever. But we still have to go in in the morning and have a smile on our face and take their vitals like you don't know anything and - that's the worst, 
the worst, absolutely worst part of the job is having to be fake and - yeah, just knowing that that's going to be the worst day of their life and there's nothing you can do about it. (FCC-13; p.3-4).

Another participant stated that she felt like she was betraying a patient's mother when she withheld the information from her. She had to lie and tell the parent that she did not know the results. This placed this nurse in a difficult situation (morally and ethically) because as a pediatric oncology nurse, she tried to create a therapeutic relationship with the patient-family. When she had to lie she felt as though this undermined the relationship.

I was in a time where a kid who had relapsed and I knew but the family didn't know yet and I had them all day and mom was asking me what are his counts and all this stuff and I didn't feel I was in the place to say that because that was the doctor's role and we knew that a family meeting was going to take place eventually and stuff and I felt in a totally hard spot because in a way I wanted to tell her but in a way I really didn't want to tell the mom because I - yeah, it didn't feel like my information to share but yet I totally felt like I was hiding something from her all day until the doctors finally got around to talking to her, and I felt that was really hard cause I didn't want her to feel like I was betraying, like not telling or hiding something from her. (FCC-17; p.7-8).

\section{Not Passing Along Information}

\section{From Nurse to Nurse}

The participants indicated that an important aspect of FCC occurred when nurses passed along information about the patient-family from nurse to nurse. When this did not happen, the nurse coming on shift was not privileged with valuable information. This became frustrating for the parents because they were asked repeatedly for the same information. As a result, the parents did not trust the nurses; they felt that they had to keep their eyes and ears open to ensure the nurses did not make a mistake.

I think that's more communication between nurses and I think that sometimes that does lack, but I think that lacks no matter where you work. So yeah, I think that that's 
also a really hard part of family centered care is things don't get passed on. And it's frustrating on our part when the parents are like, 'We've told them this so many times. He needs his meds warmed up before you give them through his NG [naso-gastric tube]. We've told them that so many times and you guys aren't passing it on.' So it is frustrating... So I think, yeah, that totally breaks FCC but I think it's our lack of communication between nurses. I think it's like our report to each other and just also after 12 hours of work you just want to go home. (FCC-12; p.11).

In the following remarkable excerpt, a nurse called the unit to share valuable information regarding a patient's diet. This nurse indicated that it was extremely valuable to have specific knowledge about patient-families because then nurses can tailor their care accordingly. When nurses do not have such information they are not able to tailor the care, and therefore, may not provide quality nursing care.

There's one nurse that really she's incredible, she phoned on her day off one day, not that I think it is our responsibility because we have to make a separation, but she phones and she's like, oh so and so in this room, they don't have parents with her at all, so she you know what I just got thinking I'm eating my breakfast, and I'm just thinking nobody knows what she likes and I finally found out what she likes and so she told me and I was like ah.. I love you for saying that, you know that makes my day, I can make this child eat. (FCC-06; p.9).

\section{From Health Care Professional to Patient-Family}

Participants noted that a barrier to FCC occurred when the medical staff did not provide information to the patient-family about the condition of the patient. Participants indicated that the patient-family felt out of the loop when the medical staff did not communicate openly with them in order to keep them informed as to what was going on.

That is a complaint that you hear, that they don't know what's going on and they don't ever know what the plan is. And that has to do with two faults; bad communication on the part of the nurses in what's going on, and bad communication from our physicians. And them not asking probably because they don't know what to ask or they're nervous to ask the physician so they ask the nurse, but then the nurse doesn't pass it on so there's lots of holes in communication that can make them feel non-involved. And we do hear that a lot. So if they were present in rounds they would hear it at least. (FCC-06; p.16). 
An important part of FCC is keeping the family informed about test results, treatment plans and treatment options. When the patient was acutely sick, the nurse focused on skill based interventions and did not have time to provide information to the patient-family. Participants indicated that this was a barrier to FCC.

I guess probably in those moments when a patient's acutely ill. Thinking back now, you know, you're so task oriented or focused and maybe not providing enough information of what was going on at that point in time. (FCC-14; p.1).

Summary. As discussed, there are many multi-faceted challenges for pediatric oncology nurses while providing FCC to the patient-family. When the participants did not have an understanding of the patient-family's culture and religious belief system they were not able to fully provide FCC. There were instances when the participants did not agree with the patient-family's decision or the participants were unable to meet the patient-family's request; this prevented them from being able to enter into a therapeutic relationship with the patient-family. Lack of time and increased acuity were barriers that every participant stated decreased their ability to provide FCC. Within the dataset, comments about the barriers and challenges to FCC were the most dense compared to other topics covered in the interviews. Therefore, pediatric oncology nurses face very real barriers and challenges when providing FCC to patient-families.

\section{Conclusion}

In this chapter, themes arising from 20 person centered interviews were presented. The majority (80\%) of the participants felt that the ACH supported FCC, while $20 \%$ stated otherwise. The participants noted that FCC involved encompassing the whole family in the care provided to the child. There were practical and functional ways the participants established FCC in their practice. More specifically, there were approaches 
that the participants used to provide exceptional FCC that went above and beyond baseline FCC. Unfortunately, providing FCC to children with cancer and their families did not come without a challenge; there were very real barriers that prevented them from doing so. In the next chapter, I discuss how the findings from this study compare with previous research. 


\section{CHAPTER FIVE: DISCUSSION}

In this chapter, I discuss the findings in relation to the current literature on FCC. First, a brief overview of the literature review, framework, study method and findings are presented. I also engage in an evaluation of the theoretical framework that was used to guide this study. In terms of the findings, major strategies that promote the implementation of FCC in nursing practice as well as barriers to FCC are presented. Furthermore, FCC is understood and presented within the context different perspectives. These include: Systems-Based, Nursing Care, and Both Nurse and Patient-Family. Barriers and challenges to FCC are addressed in depth, and recommendations about FCC in the midst of such barriers are presented. Limitations associated with this study are reviewed at the end of the chapter.

Overview of the Study

FCC is at the core of pediatric nursing. Understanding and acknowledging that parents are the experts in their child's health is central to providing FCC. Unfortunately, not all pediatric nurses incorporate FCC into their practice because there are barriers and challenges that prevent them from doing so. Such barriers and challenges include; the prevalence of the traditional medical model, the nurse's lack of understanding about FCC, time pressures on nurses, intrusive medical technology, staff shortages, an increase in the cultural diversity of the patient-family, and increased job stress.

Children with cancer and their families experience tremendous stress as a result of the various treatment regimens and the plethora of tests they must undergo. Caring for such families can be complicated because they require specific skills and expertise. FCC 
among pediatric oncology nurses is germane because the families are extremely involved in the care that their children receive.

This study used person-centered interviewing and was guided by the conceptual framework of FCC as described by the Institute of Family-Centered Care (2008). The population of interest was registered nurses and nurse practitioners from the Alberta Children's Hospital (ACH) in Calgary Alberta who practiced within the pediatric oncology program; consisting of the inpatient unit and outpatient clinic. The study included a sample of 20 nurses; 3 nurse practitioners and 17 registered nurses. Data saturation was attained after the 14 th as no new themes were distinguished. However, the subsequent 6 interviews contributed to the validation of the established themes and added more texture to them.

The participants moved from the respondent role to the informant role as described by Levy and Hollan (1998). In the respondent role the participant's spoke to their experiences practicing within the FCC framework. In the informant role, the participants answered questions about FCC; such as, provided definitions.

Data for this study were collected directly from the nurses during the 2009 academic year. This sample was a convenience sample, meaning the participants who met the inclusion criteria were recruited based on their availability (Polit \& Hungler, 1999). See Table 4.2 for demographic information of the participants.

A poster (see Appendix A) with pamphlets (see Appendix B) was placed in the break room, report room and staff room on the in-patient oncology unit and the outpatient oncology clinic. The pamphlets offered a brief description of the study and inclusion criteria, as well as my name and contact information. Nurses who were interested in 
participating in the study contacted me directly. The first 20 nurses who responded were added to the list of interviewees. The interviews took place on the unit in the Nurse Resource Room and the Team Meeting Room while the participants were on shift.

The nurses on the in-patient unit and the out-patient clinic were very supportive and responsive to this study. There was no difficulty in finding participants to enroll in this study. They called or e-mailed me to set up a time for the interview, and were enthusiastic to share their experiences about FCC. More than 20 nurses offered to participate, but I had to turn down willing participants as the set sample size for this study was achieved. Moreover, saturation was deemed to have occurred after the 14th interview. Within the oncology program at the $\mathrm{ACH}$, much time and energy is spent on medical and scientific research within this program. The nurses appeared excited to participate in research that was nursing focused because this was a rare occurrence within this program.

The participants were engaged in semi-structured, person-centered interviews. Each interview lasted approximately one hour. Informed consent (see Appendix C) and demographic data (see Appendix D) were obtained before the interviews began. The participants were asked a set of open-ended questions and were encouraged to share their experiences and explain the extent to which they practiced FCC. An interview outline (semi-structured guide) was used to provide the interview with some structure (see Appendix E). The interview guide was created from the core concepts of FCC arising from the Institute of Family-Centered Care (2008). I was attentive in allowing the interviews move in directions not expected. The interviews were digitally recorded and transcribed verbatim. 
Thematic analysis was conducted to analyze the data for this study (Polit \& Hungler, 1999). The transcripts were read carefully with an eye to identify concepts and clusters of concepts (Polit \& Hungler, 1999). The related concepts were grouped together to constitute themes and subthemes (Polit \& Hungler, 1999). The data were then re-read for content and their fittingness to the themes was reviewed.

The themes that were generated from the interview data were grouped into five major domains: How does the ACH support FCC?, Participant's Definition of FCC, Establishing FCC, Enhancing FCC, and the Barriers and Challenges to Providing FCC (see Table 4.1). The clear majority of participants (80\%) identified that the ACH supported FCC, while $20 \%$ stated otherwise.

The participants stated that FCC involved encompassing the whole family in the care provided to the child. There were practical and functional ways the participants established FCC in their practice. More specifically, there were approaches that the participants used to provide exceptional FCC. Unfortunately, providing FCC to children with cancer and their families did not come without a price; there were very real barriers and challenges that prevented them from doing so.

\section{Usefulness of the Theoretical Framework}

The conceptual framework used to guide the interviews was created from the core concepts of FCC that arose from the Institute of Family-Centered Care (2008) (see Chart 2.1). This framework was useful in providing direction to the interviews and was utilized to formulate questions to ask of the participants. As discussed, there were many challenges and barriers to providing FCC that were not mentioned in the framework. For example, lack of time and not understanding the patient-family's specific culture. 
Furthermore, the theoretical framework from the Institute of Family-Centered Care (2008) did not address or provide solutions for health care personal on how to overcome such barriers and challenges.

The framework offered a general theoretical perspective regarding FCC and nurses' contributions to it. FCC, according to the Institute of Family-Centered Care (2008), is an ideal worth striving towards. Participants were well aware of the main concepts and agreed with the principles of this framework. However, there were definite barriers that prevented or hindered the nurses from providing FCC to patients and their families. As previously mentioned, such barriers and challenges are missing from the framework. Based on the findings of this study, the framework is an ideal that offers basic direction on how to provide FCC.

Implementation of FCC; Strategies and Barriers

The participants noted that there were approaches they used that aided them in providing FCC. However, there were also very real barriers in their workplace that prevented them from providing FCC. The strategies and barriers to FCC as reported by the participants are outlined in the table below (see Table 5.0). 
Table 5.0

Strategies and Barriers to FCC Among Pediatric Oncology Nurses

\begin{tabular}{|l|l|}
\hline \multicolumn{1}{|c|}{ Strategies } & \multicolumn{1}{c|}{ Barriers } \\
\hline Encompass the Whole Family & Do not Understand Patient-Family Culture \\
\hline Collaborate with the Patient-Family & Tensions between Nurse and Families \\
\hline View Parents as the Expert & Lack of Time and High Patient Acuity \\
\hline Active Listening & Limited Space and Lack of Resources \\
\hline Respect Culture and Beliefs & Lack of Education about FCC \\
\hline Teamwork with Colleagues & Difficult Working Relationships \\
\hline Nurse Patient-Family Relationship & Brave Face \\
\hline Encouraging the Cancer Community & Not Passing Along Information \\
\hline $\begin{array}{l}\text { Having the Attitude that there is Always an } \\
\text { Opportunity }\end{array}$ & \\
\hline Be Flexible: Not Dictating & \\
\hline Caring for the Parent & \\
\hline Educating the Patient-Family & \\
\hline
\end{tabular}

\section{FCC from a Systems-Based Perspective}

The system referred to in this section is the Alberta Children's Hospital (ACH) and includes managers and administrators. This perspective is important to address because it consists of personal who make executive decisions regarding the care provided to children and their families at the $\mathrm{ACH}$. Therefore, $\mathrm{FCC}$ will be discussed in regards to how the system of the ACH impacts such care.

\section{ACH Support}

Bruce and Ritchie (1997) conducted an exploratory and descriptive study of nurses' perceptions and practices of FCC. They found that their institution did not support FCC in their practice setting. In contrast, the majority of participants in this current study indicated that the ACH supported FCC within their setting, even though most of the participants noted that there was a lack of education regarding the practical implementation of FCC. 
There were no established guidelines or standards of practice regarding FCC for the nurses to follow at the ACH. This finding was also noted by Bruce and Ritchie (1997) who observed that, "there were no established guidelines or standards for its practice or provisions to provide nurses with education and opportunities to practice family-centered care," and that "simply espousing a philosophy of family-centered care does not ensure that the philosophy will be practiced" (p.220). This is true for the ACH as the importance of FCC is recognized. In fact, the hospital was designed and constructed to support a FCC approach to caring. However, the participants voiced limitations about the required skills and knowledge needed to fully implement FCC into their practice.

Although $80 \%$ of the participants noted that the ACH supported FCC within their practice setting, most of them indicated that there was a lack of education about FCC. Of the participants $(n=20)$, three had attended a family-system nursing conference at the University of Calgary and found it extremely helpful. They stated that it improved their knowledge and ability to implement FCC into their practice. According to Peterson, Cohen and Parsons (2004), from an organizational perspective, it is inadequate to endorse philosophical concepts of FCC and not dedicate resources to ensure the frontline staff have an understanding and enactment of such philosophy.

- ACH might consider initiatives to ensure that nurses are given the opportunity to formally learn about FCC and practical ways to implement it into their practice. Involving Parents in Rounds

Within FCC, health care providers ensure that families are an integral part of the team. One way to accomplish this is to include the patient-family in rounds (Griffin, 2003). The participants from this study also noted that a way to include the patient-family 
in decision making and keep them better informed was to involve them in rounds. Participants in Higman and Shaw's study (2008) indicated that including the patientfamily in rounds was a practical way to implement FCC. Meuthing et al. (2007) implemented the practice of including families of pediatric patients in rounds. They confirmed that by doing so they fostered attitudes of respect and interest in the concerns and opinions of patient-families. Also, they were better able to create relationships and partnerships with patient-families.

When Meuthing et al. (2007) first implemented this practice, there was resistance from the medical staff because they felt it would increase time and disrupt usual workflow. After including the patient-family in rounds for a short while, this resistance disappeared and the medical staff began to praise family-centered rounds because they improved clinical outcomes and fostered patient safety (Meuthing et al., 2007).

- Including the patient-family in rounds within this department would likely enhance FCC and strengthen relationships between care providers and families.

A barrier to FCC occurred when information from the medical team was not shared with the patient-family. Including the patient-family in rounds is a possible solution to this barrier because they would be present when their child's test results are discussed and treatment options are presented; thus, they would be better informed.

\section{Interdisciplinary Approach}

The participants in this study identified that a team approach was important when implementing FCC because it ensured that the patient was receiving the best care possible. Klassen et al. (2008) affirmed that "the delivery of paediatric oncology patient care requires a team approach involving an array of health professionals" (p.16). The 
participants in this study noted that it was important to collaborate with the patient-family, which was also noted by Klassen et al. (2008). Nelson and Polst (2008) implemented an interdisciplinary team approach to FCC within an adult ICU when their staff nurses indicated that they were overwhelmed with patient acuity. Subsequently, they received recognition in 2002 as a high-performing ICU from the National Coalition on Healthcare/Institute for Healthcare Improvement.

The nurses within the oncology program at the ACH have the opportunity to work with many different disciplines when providing nursing care. Study participants indicated that they understood teamwork was important when implementing FCC, but the only team members they identified were spiritual care providers, social workers, child life specialists and physicians. Pharmacists, dieticians, respiratory therapists, psychologists, occupational therapists, and physiotherapists were not mentioned, and few participants identified volunteers as important members of the health care team. Also, some of the participants noticed that they could make better use of social work and spiritual care than they currently do.

- A possible solution to the high stress levels experienced by pediatric oncology nurses entails the incorporation of other disciplines as members of the healthcare team. Relying on other disciplines and including them as part of the care team could decrease the stress on nurses caused by patient acuity and heavy work load.

According to Penkman, Scott-Lane and Pelletier (2006), children with cancer and their parents require emotional support when undergoing cancer treatment. This can be in the form of a psychosocial team comprised of social workers, clinical psychologists and child-life specialists (Penkman, Scott-Lane \& Pelletier, 2006). Such teams help mobilize 
the parent's and children's coping skills and lessen the psychological impact of cancer (Penkman, Scott-Lane and Pelleteir, 2006). The participants in this study identified that social workers and child-life specialists were a valuable part of the medical team in caring for the patient-family's emotional needs. However, they did not mention a psychologist, and there is currently no psychologist working within the program that offers services to the patient-family.

- Having a registered psychologist would be of value to the team in helping care for the patient-family's emotional well-being.

\section{Nursing Care Perspective}

All of the participants in this study stated that FCC was an important part of the care provided to pediatric oncology patients and their families. The findings from this current study align with Bruce and Ritchie's (1997) findings from their exploratory study; nurses in their study agreed that FCC was a necessity in nursing practice. Peterson, Cohen and Parsons (2004) also found that pediatric nurses agreed that elements of FCC are important in the delivery of care to patients and their families.

There were no major differences in the perceptions of FCC between inpatient nurses and outpatient nurses. This may be because all of the outpatient nurses worked as inpatient nurses at the beginning of their careers; therefore, they may have most likely shared similar experiences. No themes emerged from the data whereby the nurse practitioners had a different perspective on FCC compared to the rest of the participants, although it was evident that their roles were different in caring for the patient-family. The nurse practitioners' role included managing and developing care for the patient-family, where as the registered nurses role included providing and implementing care. 
The findings from this current study differ from those of Bruce and Ritchie (1997) because they found that age and nursing position were related to nurses' perceptions of FCC. In contrast, in this current study the participants age and education did not influence their perceptions of FCC. This finding was also established by Peterson, Cohen and Parsons (2004). The education level, clinical experience or pediatric experience of the nurses in Bruce and Ritchie's study (1997) did not influence their perceptions on FCC, and this was confirmed in this current study.

\section{Mentoring the New Nurse}

Participants noted that there was a lack of education available to them regarding the principles and practical ways to implement FCC into their practice. Higman and Shaw (2008) conducted a qualitative study to understand how well nurses felt they were able to provide FCC and the difficulties they experienced. The findings from this current study support those of Higman and Shaw (2008) as their participants stated there was a lack of adequate training in relation to FCC. Bruce and Ritchie (1997) also identified that a lack of understanding about FCC was a barrier to providing FCC among nurses.

It can be difficult for inexperienced nurses to concentrate on caring for a child while answering questions from parents (Griffin, 2003). Benzein et al. (2008) found in their descriptive study that newly graduated RNs viewed the family as a burden because they did not have time to take care of them. They also found that newly graduated RNs focused on the individual patient more than the family. The findings from this current study validate those of Benzein et al. (2008). New nurses in this current study stated that they focused most of their energy on learning and performing clinical nursing tasks and interventions, thus they were unable to focus on caring for the family. 
Benner (1984) described the concepts of novice nurse and expert nurse. The novice nurse is a beginner who has limited experience of situations in which she/he is expected to practice (Benner, 1984), and lack experience on therapeutic relationships (Godkin, 2001). The expert nurse does not rely on analytic principles to connect understanding of a situation to a suitable action; she/he has an intuitive comprehension of each situation and acts without wasteful consideration of unfruitful solutions (Benner, 1984). This was found to be true in this current study. New or novice nurses expressed that they did not have the clinical foundation to provide FCC while completing tasks because they lacked experience. Nurses described a point in time, about two years after starting their nursing careers, where they felt they could provide FCC while conducting tasks: these tasks became a means to provide FCC.

Participants in this current study indicated that the best way to educate new nurses about FCC was to demonstrate it through mentoring. They stated that active involvement and practical examples were the best ways to learn FCC, which also came through experience in interacting with patient-families. These findings confirm Higman and Shaws' findings (2008), as they discovered that the best way to learn FCC was for senior nurses to share their experiences with new nurses, and indicated that it was acquired with experience and over time. Benzein et al. (2006) also found that in order for newly graduated RNs to increase their supportive attitudes towards families, experienced nurses needed to provide them with support and guidance on doing so.

Peterson, Cohen and Parsons (2004) found that FCC education should focus on skill development with educational interventions such as interactive workshops. Such activities provide nurses with an opportunity to enhance their communication skills and 
develop professional relationships. According to Griffin (2003), mentoring, education and role-playing are beneficial ways to help new nurses develop skills on how to provide FCC. Participants in this current study indicated that the best way to 'teach' FCC is to have senior experienced nurses role model and mentor less experienced nurses regarding FCC. Also, the few nurses who did attend an interactive workshop at the University of Calgary stated it was extremely beneficial, thus, it was discovered that education also plays a role it teaching new nurses about FCC.

- It would be beneficial for the ACH to hold interactive workshops on FCC for nursing staff and to initiate mentorship programs for new nurses.

Nurse and Patient-Family Perspective

This section will address FCC from the perspective of the nurse and the patientfamily. I will discuss FCC in terms of what happens when reported care is provided to patients and their families by pediatric oncology nurses.

Not Discussing the Dying Child or the Word Cancer

The participants indicated that it was difficult to provide FCC when the parents would not permit them to talk about the diagnosis in front of their child. Also, certain parents did not want to tell their child that she/he was dying when they transitioned to palliative care. These situations were difficult for the participants because they felt that they were lying and being deceitful to the child. Dunlop (2008) described that when determining if a child is emotionally prepared to know their diagnosis, the nurse can encounter conflict, moral distress and frustration. Participants in this study experienced compounded stress: stress from dealing with parents who did not want to tell their child about her/his diagnosis, and stress from determining if the child was prepared to know 
their diagnosis as described by Dunlop (2008). Such instances were confusing and distressing for the participants in this current study.

The participants indicated that when conflict arose between the parent and nurse on what to tell the child in regards to their diagnosis, they allowed the parents to determine what information to share. It was not evident from the interviews that health care professionals provided the parents with information on how to decide what to share with the child and its implication on the emotional and psychological well-being of the child. According to Wolf (2004), children who endure life-threatening diseases have witnessed and know of other children's deaths, and most children who are dying become aware of their impending death on their own. Muris et al. (1996) noted that if the dying child is not told the truth about his or her situation, the child does not understand or know how to exercise control over their fears of dying. This negatively affects their quality of life. Therefore, it is important that healthcare professionals address the issue of not telling the child about their diagnosis with the family and come to a conclusion in the best interest of the child. Dunlop (2008) asserts that an absolute dogma regarding telling the truth does not exist, but that healthcare professionals must create an atmosphere of support and openness that encourages truthfulness.

- Supportive environments, as described, should be created in the pediatric oncology department at the ACH because the participants deeply struggled with such situations.

\section{Moral Distress}

According to Hamric, Davis and Childress (2006) moral distress is a situation where the nurse: 
knows, or believes she knows, the ethically appropriate action, but feels constrained from acting because of some obstacle inherent in the situation, such as lack of time or supervisory support, institutional or legal constraints, or physician power over nursing and nursing practice (p. 18).

Nurses who are morally distressed feel powerless and as though their 'hands are tied' because they are not able to speak up and are not in charge of the patient care situation; often the physicians exercise this privilege and power (Hamric et al., 2006). As a result, nurses become distressed because they are forced to compromise their integrity by failing to take a stand and their silence comprises the patient's care (Hamric et al., 2006).

The participants in this current study experienced moral distress in situations when they had to put on a "brave face," forced care on a child who followed the Jehovah Witness religion, and were not permitted to tell the child the truth about her/his diagnosis. In such instances, the nurses felt that their actions were not ethical, and obstacles were present that prevented them from doing what they sensed was right.

The findings from this study indicate that pediatric oncology nurses faced moral distress frequently in their practice. This is an issue that should not go unaddressed because it can cause the nurse to withdraw emotionally from patients and their coworkers, have feelings of anger, guilt, and depression, and leave their position or profession (Hamric et al., 2006).

- The nurses did not appear to be aware of any resources or programs in place within their department that would help them to deal and cope with moral distress. Therefore, this is an area that should be addressed within the pediatric oncology department at the ACH so that nursing staff can continue to provide FCC in situations where moral distress is present. 


\section{Care for Whole Family in the Context of the Patient}

Every participant in this study described FCC as being a caring process that involved the whole family. This finding corresponds with Shields, Pratt and Hunter's (2006) definition of FCC; "family-centered care is a way of caring for children and their families within health services which ensures that the care is planned around the whole family, not just the individual child/person, and in which all the family members are recognized as care recipients" (p.1318). The participants in this current study held a basic understanding that FCC meant to include the whole family in the planning and delivery of care to patient-families.

Woodgate (2006) conducted a qualitative interpretive study to understand how adolescents with cancer deal with challenges faced during their treatment. Support from the family was identified as the most helpful relationship experienced throughout their disease journey. What mattered the most to the adolescents was knowing that their families would always be there for them, and it was the support from their families that gave them the strength to carry on (Woodgate, 2006). The participants in this current study recognized the importance of the child's relationship with the family because they all identified the family as playing a supportive role in the child's life. They made it a priority to include the family in the care provided to the child.

Bjork, Nordstrom and Hallstrom (2006) found in their qualitative study that parents had an important role in the life of children with cancer while in the hospital. The child wanted to be physically and emotionally close to his or her parents (Bjork et al., 2006). The findings from this current study parallel those of Bjork et. al, (2006), as participants noted that the family played an important role in the child's life and that it 
was important to include the whole family in the care. Whenever possible, the participants supported and encouraged the family to stay with the child and be a part of the team.

\section{Nursing Presence}

"The art of pediatric oncology nursing practice includes the crucial element of nursing presence" (Cantrell, 2007). Cantrell (2007) argued that in order to maximize the impact of care provided to children with cancer, the art and science of pediatric oncology nursing practice must be evident in the various care activities provided to the patientfamily. Such activities must occur within a therapeutic relationship that is embedded in nursing presence (Cantrell, 2007).

McKivergin and Daubenmire (1994) state:

Opportunities in which to be sensitive to patients' needs are often missed and depth of therapeutic interaction is passed over in lieu of completion of tasks. This yields frustration on the part of other caregiver as well as within the patient. Desires to listen and 'be with' the patient are often overrun by demands to 'do to' the patient (p.65-66).

The participants in this current study noted that there were times when they were too busy and focused on completing tasks and interventions that they were unable to be 'present' with the patient-family. They identified that they had an internal struggle with such situations because it was important to them to create therapeutic relationships with the patient-family and care for their emotional and spiritual well-being.

McKivergin and Daubenmire (1994) noted that when the nurse is present with the patient, she/he is able to provide support and help the patient-family gain more insight into the nature of the disease. Also, the parents are then able to become partners with the medical staff in making life choices, which brings about the highest level of health and 
healing (McKivergin \& Daubenmire, 1994). The findings from this current study parallel those of McKivergin and Daubenmire as the participants indicated that it was important to provide the patient-family support during their disease journey and noted that it was essential to partner with the patient-family in making choices.

Doona, Haggerty and Chase (1997) defined presences as:

An intersubjective encounter between a nurse and a patient in which the nurse encounters the patient as a unique human being in a unique situation and chooses to spend her/himself on the patient's behalf. The antecedents to presence are the nurse's decision to immerse her/himself in the patient's situation and the patient's willingness to let the nurse into that lived experience. As a consequence of nursing presence, both the nurse and the patient are changed. Both are affirmed as unique human beings, and the nurse is affirmed as a professional and the patient as a person in need (p. 12).

The participants in this study did not mention that they were specifically implementing nursing presence into their practice, but through their descriptions of FCC it was evident they were. They all mentioned the importance of viewing each patient individually and as a human being. They all stated that it was important to spend time with the patient-family and that they all valued the connections and relationships that were formed with the patient-family. Some participants indicated that they were better people as a result of their time spent with the patient-family because it caused them to better appreciate their own lives. Also, they all indicated that when they knew they were making a difference in the lives of the patient-family they felt satisfaction within themselves and with their jobs.

Godkin (2001) stated that bedside presence occurs when anyone is determined to establish rapport and make oneself available to another. It involves being present and freely interacting with the patient; it is a gift (Godkin, 2001). This was mentioned specifically by one participant in this study. She stated that making herself available was giving a gift of herself to the patient-family. 
Woodgate (2006) found through "the act of 'being there' that adolescents experienced a sense of presence with the individual in the relationship and made the adolescents realize what really was important in life" (p. 126). The presence of a person being there for the adolescent with cancer was comforting and helped them to cope with their situation. This was identified by the participants in this current study because they recognized the value of being present with patients. The participants felt an internal struggle when they were pressed for time and could not be with the patient-family because they knew that their presence was healing and comforting to the patient-family.

Woodgate (2006) also found that being there to comfort the adolescent was important because it helped them feel better, both physically and mentally. The adolescents in Woodgate's study (2006) stated that the simple act having their hand held was associated with the act of comforting. This current study validates Woodgate's findings (2006) as the participants recognized that the small acts they conducted, such as covering the patient with a warm blanket, made a big difference in the patient's life.

\section{Nurse Patient-Family Relationship}

MacKean, Thurston and Scott (2004) conducted a qualitative study to develop a conceptualization of FCC grounded in the experiences of families and health care providers. The health-care providers and the families in their study identified the relational competencies of the health-care provider as an important contributing factor to the family's experience. The relational competencies that received the most recognition from health-care providers were: being open to discussion and negotiation, communicating in a direct and honest manner, listening, valuing parents' input and being informative (MacKean et al., 2004). Findings from this present study lend support to 
those of MacKean et al. (2004) as participants identified that important aspects of the nurse patient-family relationship were being flexible and open to negotiation, being honest, listening, acknowledging the parent as the expert, including the family in decision making, educating the family and passing along information.

The overall findings from MacKean et al.'s (2004) study support the “importance of the relationship component of family-centered care, specifically the development of true collaborative relationship between families and health-care providers" (p. 81). The findings from this current study parallel MacKean et al.'s (2004) findings in that the participants identified the relationship with the patient-family and collaboration as important aspects in the implementation of FCC.

Hutchfield (1999) found that an important attribute of FCC is the relationship between the family and nurse, and is based on respect and open communication. Similarly, participants in this current study identified the nurse patient-family relationship as beneficial in the implementation of FCC. Also, open communication was deemed important. Hutchfield (1999) found that co-operation, collaboration and negotiation are not always evident in care provided to sick children. Bruce and Ritchie (1997) found that nurses were unlikely to include activities that would increase and facilitate collaboration between nurses and parents. In contrast, participants in this current study noted that it was important for them to collaborate with the patient-family and to negotiate care within the FCC framework.

Johnson and Lindschau (1996) found that nurses had reservations about the parent's ability to conduct complex aspects of their child's care and that they were unwilling to give up control. In this current study, participants recognized the need and 
importance of giving patient-families control and power over their care. The only time that the patient-family was not involved in care was when it became unsafe for the patient. It was not indicated as an unwillingness to give up control by the nurse, rather ensuring safety for the child. The parents were taught how to conduct complex tasks such as central venous catheter care and encouraged to do such care at home unsupervised.

Widger and Wilkins (2004) established that parents need to be involved in the care of their child because it gives them a sense of control. The findings from this current study are aligned with those of Widger and Wilkins (2004). It was noted that the parents lose a sense of control when their child is diagnosed with cancer. Participants also noted the importance of instilling a sense of control in the patient-family as a means to help them cope and live through their tragic situation.

Benzein, Johansson, Arestedt and Saveman (2008) observed that,

Viewing families as a resource in nursing care and as their own resource means valuing the presence of families in nursing care, inviting them to take part in the care of their family member, creating a good family-nurse relationship, and considering family members as cooperative/collaborative partners (p.172).

This observation received support from this current study. In most instances, participants viewed parents as experts, therefore, they were incorporated as a valuable resource and their presence was valued. The participants encouraged the family to make decisions; therefore, they invited them into the care of the child. Teamwork with the patient-family was important to the participants and the patient-family was viewed as a cooperative/collaborative partner. An exception to this occurred if nurse had different values from the patient-family or the family was difficult.

Shields, Pratt and Hunter (2006) conducted a systematic review of qualitative studies on FCC. They found that the freedom of negotiation between the patient-family 
and nursing staff was key to the success of interactions. The participants in this current study noted that it was important to be flexible and open to negotiations when caring for children with cancer. When they gave the patient-family options and the ability to be part of the decision making team, their relationship was more trusting.

Woodgate (2006) discovered that support from the health care team was of great importance to adolescents with cancer because they referred to them as their "second family." They had much love and high esteem for the health care team (Woodgate, 2006). The participants in this current study recognized that they played a supportive role in the patient-family's life when they went through their cancer experience. This recognition led them to realize the value of FCC in pediatric oncology nursing practice and encouraged them to create relationships with the patient-family.

\section{Cultural Diversity of the Patient-Family}

Pergert, Ekblad, Enskar and Bjork (2007) conducted a grounded theory study among pediatric oncology nurses to reveal obstacles to transcultural caring relationships. Linguistic diversity was the primary obstacle to developing a transcultural relationship because the health care provider could not communicate directly with the family, which led to difficulties in sharing information. When the healthcare providers were unable to communicate with the patient-family, misunderstandings and conflicts to daily care of patients occurred (Pergert et al., 2007). The use of an interpreter meant that the health care staff lost control over the information that was given, which resulted in uncertainty if the information was accurate and complete.

The findings from this current study echo those of Pergertet et al. (2007). Participants noted that language barriers undermined their ability to provide FCC because 
they were unable to communicate information to the family about their child's condition. Also, the use of interpreters was difficult because the nurses did not know if the patientfamily fully understood what was being conveyed to them. As demonstrated, conflicts occurred between nurses and the patient-family because of a lack of communication in light of language barriers.

Pergert et al. (2007) also found that the health care provider and the patient-family had different views and practices regarding how to communicate and interact, such as nonverbal communication and when to discuss something (i.e., timing). This current study supports the findings of Pergert et al. Participants noted that given their lack of understanding of certain cultures, they could undermine FCC through their actions. They also described situations when they did not agree with the decision the family made, but were unsure if they could display/demonstrate their disagreement.

Pergert et al.(2007) noted that other obstacles to the transcultural relationship included: differences in emotional expression, truth telling, gender roles, racism and prejudice and inequity in care. None of these obstacles were noted by the participants in this current study, with the exception to truth telling as demonstrated by the theme 'brave face.' It was strongly expressed by the participants that they did not form judgments against patients and their families if they had different values and beliefs, but that it was imperative to treat such families with respect. Participants noted that the nurse needed to ask questions and learn about the family's culture, values and beliefs, and incorporate such knowledge into this care. The participants from this current study appeared to be more willing to provide care to patients from different cultures than the participants in Pergert et al.'s (2007) study. 
In Bruce and Ritchie's study (1997), the nurse's ranked respecting family individuality as the highest element of FCC. This current study also lends support to these findings as participants stated that nurses must learn to respect the specific cultures, values, and beliefs of each individual patient-family. They should not assume or form judgments about patient-families who have values and beliefs different than their own. The participants noted it was important to recognize that there are cultures within a culture, and they must approach each situation with an open mind and not assume a textbook definition of the patient-families culture.

\section{Educating the Patient-Family}

According to Sobo (2004) pediatric cancer treatment involves toxic pharmaceuticals and complex and changing medication regimens; miscommunication between the nurse and patient-family can cause medication errors and negatively impact adherence. The participants in this study addressed this issue when they discussed the importance of education. They recognized it was vital to inform and educate the patientfamily because they were highly involved in the care.

Holm, Patterson and Gurney (2003) conducted a qualitative study to address ways parents participate in the medical care of their child with cancer. Parents identified that it was important to be educated about their child's diagnosis and treatment in order to be advocates. Parents gathered information from the Internet, books, and healthcare professionals. By becoming educated, the parents were able to participate in decision making more effectively (Holm et al., 2003). In this current study the participants noted that educating the parents was a way to empower them to become advocates and participate in the decision making process for their child. 


\section{Barriers at all Levels}

Craft-Rosenberg, Kelley and Schnoll (2006) conducted a qualitative study to describe the views of FCC reported by practitioners serving children. They found obstacles to providing FCC were "difficult" families, ethnic diversity and structural impediments, such as lack of time. All of these barriers were noted in this current study. Difficult families prevented the participants from creating therapeutic relationships with the patient-family. Ethnic diversity of the patient-family hindered the participants from providing FCC because they did not know what was appropriate within the context of patient-family's cultural beliefs. Every participant noted that lack of time was a barrier to FCC because they were not able to spend the time they needed to be present with the patient-family.

Ahmann (2003) stated that not keeping the family well informed can cause them to have feelings of anger, and conflict between the family and nurse can arise. The participants in this current study noted that this was a barrier to FCC because keeping the family fully informed enabled them to be collaborative partners in care. When the family was not informed they became upset and there was tension between the nurse and family.

In times of high acuity, meeting the needs of the family in addition to caring for the patient is difficult (Griffin, 2003). This was noted by the participants in this current study. When the patients were acutely ill, the participants were unable to take time to provide quality FCC because their focus was solely on accomplishing tasks in order to provide safe care. Thus, they did not have time to consider the needs of the family.

"The many cultural and societal variations among families can increase the difficulty in identifying who is a child's legal guardian" (American College of 
Emergency Physicians, 2006, p.633). This was noted to be a barrier to FCC in this current study because in such situations the participants identified that they could not be open and honest with all family members, or freely include all family members in the care of the child. The participants had to focus much of their time and energy on identifying who the legal guardian was and who they could share confidential information with, rather than meeting the holistic needs of the patient-family.

Bruce and Ritchie (1997) found that the hospital system did not plan services that accommodated language differences. In this current study, the participants identified that language was a barrier to providing FCC when the patient-family spoke a different language than the nurse. They did not mention that the ACH's translation services helped in overcoming this barrier.

- This is an area that warrants attention. Services should be created or enhanced to help healthcare professionals communicate with patient-families who speak diverse languages.

The $20 \%$ of the participants who stated the ACH did not support FCC within their setting based their perspective on three main reasons. First, there was no formal education that equipped the frontline staff with practical knowledge of FCC. Second, there was an inconsistency in staff from shift to shift who cared for the patient-family, which prevented nurses from creating therapeutic relationships. Third, there were budget constraints that prevented the unit from having adequate staff to care for the acutely ill patients. These three explanations were discussed and presented as barriers to providing FCC in this practice setting by all participants. Therefore, even though $80 \%$ of the 
participants stated that the ACH does support FCC, they noted that barriers still existed to the implementation of FCC.

Is FCC the Solution? The Ideal, Reality and Making Sense of it all

Shields, Pratt, Davis and Hunter (2007) conducted a Cochrane Review to assess the effects of FCC models for children in hospitals compared to professionally-centered models. No studies were found to meet their inclusion criteria, therefore, it was concluded that no analysis could be undertaken since no quality quantitative research about FCC has been conducted. Thus, no one knows or has evidence if FCC truly works in the pediatric care setting.

Bruce and Ritchies' findings (1997) indicate that FCC exists among pediatric nurses, but is practiced inconsistently. "The nurses reported that their everyday work did not match their perception of the activities necessary to provide family-centered care" (Bruch \& Ritchie, 1997, p.217). Peterson, Cohen and Parsons (2004) found that nurses agreed that elements of FCC are necessary in their care to children and families, but that they were not always reflected in their practice.

After conducting a systematic review, Shields, Pratt and Hunter (2006) concluded that more research is needed to ensure that FCC is being implemented correctly. Findings from Bruce and Ritchie's (1997) study indicated that nursing practice "continues to reflect inconsistencies in the level of desired family-centered care of children and their families" (p.219). Peterson et al. (2004) found that "nurses both understand and believe in the value of family-centered care yet cannot seem to execute the concepts when caring for children and their families" (p. 426). 
As shown, there is no solid evidence that demonstrates FCC works: only evidence that suggests it is not being implemented into pediatric nursing as intended. Pediatric nurses acknowledge that FCC is important, but always seem to come against barriers and challenges when attempting to practice with the framework. This was evident in this present study. The participants recognized the importance of FCC, but the implementation of FCC in their practice setting was hindered by many barriers and challenges. Barriers included: inadequate translation services, lack of understanding regarding the patient-family's culture, lack of time, working short staffed, and the new nurse.

When engaging in the data analysis for this study, the barrier section was the most dense. Thus, FCC was deemed important and valuable within pediatric oncology nursing, but it did not come without considerable barriers and factors that undermined its implementation. Therefore, should we continue to position FCC as the solution to providing quality care to pediatric patients and their families?

Carter (2008) argued this point in her commentary on Shields, Pratt and Hunters systematic review of qualitative studies. She stated that pediatric nurses see FCC as intrinsically good, and it has become a part of the collective psyche. Despite the fact that FCC has been welcomed with open arms by academics, practitioners and educationalists alike, there is little clear evidence that demonstrates it actually works (Carter, 2008). "We all believe that it is good but the studies consistently address and explore the ways in which we are not achieving it" (Carter, 2008, p. 2092). According to Carter (2008), a more honest approach needs to be considered because one size cannot fit all; we should not continue to embrace and engage in an approach that is reported to not be working and 
still requires more research after all these years. FCC is complex, dynamic, relational and subject to the influence of internal and external forces (Carter, 2008).

A working and operational reality of FCC exists among pediatric oncology nurses. This form of FCC is characterized by flexibility, adaptability and recognition of the barriers to FCC. It gives nurses the freedom to provide quality FCC when time permits and nurses are able to be present with acutely ill patients and their families. This working definition of FCC encourages nurses to provide education that meets the specific needs of the patient-family. It is a flexible and adaptable working model that allows nurses to decide as a practitioner what aspects of FCC are important for what patient-families: it does not expect nurses to practice a one size fits all philosophy of FCC.

\section{Recommendations}

Much of the research conducted on FCC, as discussed in Chapter 2, has focused on the lack of its implementation within nursing practice and does not provide practical strategies on how to improve its implementation. Rather than praising nurses for implementing FCC in their practice to the best of their ability given their practice stressors, research has concentrated on findings that demonstrate pediatric nurses lack FCC in their practice. Instead of concluding that the participants in this study need to strive harder to implement FCC into their practice, it has been recognized that they all value FCC and do their best implement it.

Therefore, it is recommended to embrace a practical philosophy of FCC that is adaptable and can accommodate each caring situation, patient-family and nurse. A philosophy that takes into account and embraces the strengths of each nurse and patientfamily with flexibility in the delivery and adaptability in its evaluation. 
The current philosophy of FCC is rigid and inflexible. It has a set of key concepts that are expected to be implemented perfectly in every care situation. As noted by the participants in this study, FCC is valuable to pediatric oncology nursing. Its philosophy has a set of ideals that provide general direction for nursing care. However, it does not come without challenges and barriers, which can be distressing for nursing staff. Therefore, it is recommended that a more flexible and working approach to FCC be implemented. One that works like a rubber band; where it can be bent and shaped to fit the situation at hand.

Upon reading over the transcripts I found that there were situations where the participants lacked FCC in their practice due to barriers and challenges. I realized that many of the barriers and challenges are not going to dissipate anytime in the near future. The barrier to FCC that was reported by all participants and was the most disabling was lack of time and being too busy. Given the current situation of our health care budget, there is no end in sight in regards to this barrier. As well, there is no money in the budget to create and hold education sessions on FCC. Therefore, in order to implement FCC in the midst of these barriers, we must allow FCC to be functional in an operational capacity. To recognize its strengths, be aware of the barriers and challenges, and allow it to fail and succeed where possible.

Thus, it is recommended to embrace a philosophy of FCC as stated by the Instituted of Family-Centered Care (2008) with the following additions. Each nurse is allowed and encouraged to focus on different aspects of FCC as their strengths, values and abilities equip them to do so. As a result of the barrier lack of time and acutely ill patients, it is noted and accepted that there will be situations where it is not possible to 
fully implement FCC. Due to the various personalities of the nurse, patient and families, there will be certain nurses and families that get along well, and some that do not. New nurses will not be able to practice FCC successfully at first, because they will have to become comfortable with basic nursing tasks before they can focus on the nurse-patient relationship.

The data analysis demonstrated that the participants in this study have a very good understanding FCC and highly value it in their practice. They indicated that they use a variety of strategies in their practice to implement FCC. Given that they are imbedded in this practice environment; I believe that they hold the best understanding and knowledge on how to implement FCC in pediatric oncology nursing practice. The strategies that they use include: including the whole family, collaborating, viewing the parent as the expert, active listening, respecting culture and beliefs, teamwork with colleagues, nurse patientfamily relationship, encouraging the cancer community, always looking for an opportunity, being flexible, caring for the parent and educating the patient-family. Therefore, it is recommended for pediatric oncology nurses to embrace such strategies in order to practice under the philosophy of FCC given the current challenges and barriers.

\section{Practice Administrative Recommendations}

Basic recommendations include creating a system for the physicians to share the results from various procedures and tests in a timely manner to prevent the nurses from having to put on a 'brave face.' It is advised to create and implement interventions, based on current research and literature, in regards to situations when the family does not want to tell their child that they have cancer. 
Other more basic recommendations include the following. The department of oncology at the $\mathrm{ACH}$ could initiate system changes in regards to incorporating the patient-family in rounds. This would increase patient safety and allow the patient-family to be better informed. Nursing management might give consideration to mentorship programs where junior staff can be mentored by senior staff. Nurse educators could include sessions on FCC in their education days that discuss the strategies on implementing FCC. Open discussions could be held among nursing staff where they can share their experiences of FCC and learn from others. Also, support sessions and services where pediatric oncology nurses can discuss the moral distress they experience at work would be greatly beneficial to the staff.

\section{Limitations}

Although the participants were eager to participant in this study, it does not ensure that the sample is representative of the whole population. It is possible that the participants in this study volunteered to participate because they were passionate about FCC. Therefore, I may have missed out on nurses who were not supportive of FCC. I may hold biases and perspectives on the philosophy of FCC based on my strengths, values and beliefs, which could have altered the data analysis and themes extracted. Also, the patient-families were not included in this study; therefore, their valuable perspective was not included. The method of person-centered interviewing was beneficial in that it aided in providing a well rounded explanation of the phenomenon. However, this method had limitations; such as, I was not able to visually observe the participants providing care, I was unable to fully explain the lived experiences of such caring encounters and I was not able to demonstrate that FCC is actually effective in caring for children with cancer. 
Finally, it should be noted that the themes pulled out from the data are interpretations that I myself made, and therefore, could be interpreted differently among different persons.

\section{Future Research}

It would be valuable to gain a deeper understanding of FCC by conducting further research among the patients and their families. Research regarding strategies to work with parents in openly telling their children that they have cancer would be beneficial. Research into mentorship programs for newly graduated pediatric oncology nurses would be of assistance for newly graduated nurses. Also, it would be advantageous to evaluate the process of including parents in team rounds. 


\section{References}

Ahmann, E. (2003). Facing challenges to family-centered care II: anger in the clinical setting. Pediatric Nursing, 29(3), 212-214.

Allen, R. I., \& Petr, C. G. (1996). Towards developing standards and measurements for family-centered practice in family support programs. In Singer, G., Powers, L., \& Olson, A. (Eds.) Family support policy and american's caregiving families: innovations in public private partnership (p. 57-86). Baltimore, MD: Brookes.

Alsop-Shields, L., \& Mohay, H. (2001). Issues and innovations in nursing practice. John bowlby and james robertson: theorists, scientists and crusaders for improvements in the care of children in hospital. Journal of Advanced Nursing, 35(1), 50-58.

American Academy of Pediatrics. (2003). Family Pediatrics Report of Task Force on the Family. Pediatrics, 111(6), 1541-1571.

American College of Emergency Physicians. (2006). Policy Statement. Annals of Emergency Medicine, 48(5), 643-645.

Baker, S. (1995). Family centered care: a theory practice dilemma. Paediatric Nursing, $7(6), 17-19$

Begun, A. L., (1996). Family systems and family-centered care. In, Rosin. P., Whitehead, A., Tuchman, L., Jesien, G., Begun, A., \& Irwin, L. (1996). Partnerships in family-centered care a guide to collaborative early intervention. Baltimore, MA:

Paul H. Brookes Publishing Co., Inc.

Benner, P. (1984). From novice to expert: Power and excellence in clinical nursing practice. Reading, MA: Addison-Wesley. 
Benzein, E., Johansson, P., Arestedt, K. F., \& Saveman, B. (2008). Nurses attitudes about the importance of families in nursing care. Journal of Family Nursing, 14(2), 162180.

Björk, M., Nordström, B., Hallström, I. (2006). Needs of young children with cancer during their initial hospitalization: an observational study. Journal of Pediatric Oncology Nursing, 23(4), 210-219.

Björk, M., Wiebe, T., \& Hallström, I. (2005). Striving to survive: families’ lived experiences when a child is diagnosed with cancer. Journal of Pediatric Oncology Nursing, 22(5), 265-275.

Bradshaw, M., Coleman, V., \& Smith, L. (2003). Interprofessional learning and familycentered care. Paediatric Nursing, 15(7), 30-33.

Bricher, G. (1999). Paediatric nurses, children and the development of trust. Journal of Clinical Nursing, 8(4), 451-458.

Brown, J., \& Ritchie, J. A. (1990). Nurses' perceptions of parent and nurse roles in caring for hospitalized children. Children's Health Care, 19, 28-36.

Brown, W., Pearl, L. F., \& Carrasco, N. (1991). Evolving models of family-centered services in neonatal intensive care. Children's Health Care, 20, 50-55.

Bruce, B., \& Ritchie, J. (1997). Nurses’ practices and perceptions of family-centered care. Journal of Pediatric Nursing, 12(4), 214-222.

Campbell, C. (2003). Anti-oppressive theory and practice as the organizing theme for social work education: the case in favour. Canadian Social Work Review, 20, 121125.

Canadian Cancer Society. (2008). Canadian Cancer Statistics 2008. Retrieved on August 
10, 2008 from http://www.ncic.cancer.ca/vgn/images/portal/ cit_86751114/14/35/195991821ncic_stats2004_en.pdf

Cantrell, M. A. (2007). The art of pediatric oncology nursing practice. Journal of Pediatric Oncology Nursing, 24(3), 132-138.

Carter, B. (2008). Commentary on shields 1. pratt j \& hunter j (2006) family centered care: a review of qualitative studies. Journal of clinical nursing 5, 1317-23. Journal of Clinical Nursing, 17(15), 2091-2093.

Chan, N., \& Kennedy, L. (2008). Pediatric Inpatient Unit 1 Alberta Children's Hospital Patient Family Feedback Survey Roport July 2008. Alberta Children's Hospital Decision Support Research Team.

Clarke, J. N., \& Fletcher, P. (2003). Communication issues faced by parents who have a child diagnosed with cancer. Journal of Pediatric Oncology Nursing, 20(4), 175191.

Craft-Rosenber, M., Kelley, P., \& Schnoll, L. (2006). Family-centered care: practice and preparation. Families in Society, 87(1), 17-25.

Coyne, L. (2006). Children's experiences of hospitalization. Journal of Child Health Care, 10(4), 326-336.

Creswell, J. W. (1998). Qualitative inquiry and research design: Choosing among five traditions. Thousand Oaks, CA: Sage.

Deatrick, J. A. (2006). Family Partnership in Nursing Care. In Craft-Rosenberg, M., \& Karjicek, M. J. (2006). Nursing Excellence for Children \& Families. (pp. 41-55). New York: Springer Publishing Company.

Denboba, D., McPherson, M. G., Kenney, M. K., Strickland, B., \& Newacheck, P. W. 
(2006). Achieving family and provider partnership for children with special health care needs. Pediatrics, 118(4), 1607-1615.

Dokken, D., \& Ahmann, E. (2006). The many roles of family members in "familycentered care" - part I. Pediatric Nursing, 32(6), 563-565.

Doona, M. E., Chase, S. K., \& Haggerty, L. A. (2007). Nursing presence as real as a milky way bar. . Journal of Holistic Nursing, 17(1),54-70.

Doona, M. E., Haggerty, L. A., \& Chase, S. K. (1997). Nursing presence: An existential exploration of the concept. Scholarly Inquiry for Nursing Practice: An International Journal, 11, 3-16.

Dunlop, S. (2008). The dying child: should we tell the truth? Paediatric Nursing, 20(6), $28-31$.

Dunst, C. J., Trivette, C. M., \& Deal, A. G. (Eds.). (1994). Enabling \& empowering families: principles \& guidelines for practice. Cambridge, MA: Brookline Books.

Epps, S., \& Jackson, B. J. (2000). Empowered families, successful children: Early intervention programs that work. Washington, DC: American Psychological Association.

Fochtman, D. (2006). The concept of suffering in children and adolescents with cancer. Journal of Pediatric Oncology Nursing, 23(2), 92-102.

Friedman, M. M., Bowden, V. R., \& Jones, E. G. (2003). Family nursing, research, theory, and practice. Upper Saddle River, NJ: Prentice Hall.

Galvin, E., Boyers, L., Schwartz, P., Jones, M., Mooney, P., Warwick, J., \& Davis, J. (2000). Challenging the precepts of family-centered care: testing a philosophy, Pediatric Nursing, 26(6), 625-636. 
Gibson, F., Mulhal, A. B., Richardson, Al, Edwards, J. L., Ream, E., \& Sepion, B. J. (2005). A phenomenologic study of fatigue in adolescents receiving treatment for cancer. Oncology Nursing Forum, 32(3), 651-660.

Godkin, J. (2001). Healing Presence. Journal of Holistic Nursing, 19(1), 5-21.

Griffin, T. (2003). Centered care II: anger in the clinical setting. Pediatric Nursing, 29(3), 212-214.

Guba, E. G. \& Lincoln, Y. S. (2004). Competing paradigms in qualitative research: Theories and issues. In S. Nagy Hesse-Biber \& Leavy (Eds.), Approaches to qualitative research: A reader on theory and practice (p 17-38). New York, NY: Oxford University Press.

Hamric, A. B., Davis, W. S., \& Childress, M. D. (2006). Moral distress in health care professionals: what is it and what can we do about it? The Pharos, Winter.

Harbaugh, B., Tomlinson, P. S., \& Kirschbaum, M. (2004). Parents' perceptions of nurses' caregiving behaviors in the pediatric intensive care unit. Issues in Comprehensive Pediatric Nursing, 27, 163-178.

Hart, A., Freeman, M. (2004). Health 'care’ interventions: making health inequalities worse, not better? Journal of Advanced Nursing, 49(5), 502-512.

Hedstrom, M., Haglund, D., Skolin, I., \& von Essen, L. (2003). Distressing events for children and adolescents with cancer: child, parent and nurse perceptions. Journal of Pediatric Oncology Nursing, 20(3), 120-132.

Hemmelgarn, A.L., Glisson, C., \& Dukes, D. (2001). Emergency room culture and the emotional support component of family-centered care. Children's Health Care, 30(2), 93-110. 
Hesse-Biber, S. N., \& Leavy, P. (2004). Approaches to qualitative research: a reader on theory and practice. Oxford, NY: Oxford University Press

Higman, W., \& Shaw, K. (2008). Nurses' understanding about the delivery of family centered care in the neonatal unit. Journal of Neonatal Nursing, 14, 193-198.

Holm, K. E., Patterson, J. M., \& Gurney, J. G. (2003). Parental involvement and familycentered care in the diagnostic and treatment phases of childhood cancer: results from a qualitative study. Journal of Pediatric Oncology Nursing, 20(6), 301-313.

Hopton, J. (1997). Towards anti-oppressive practice in mental health nursing. British Journal of Nursing, (6)15, 874-878.

Humphrey, E. (1991). Searching for life's meaning: A phenomenological and heuristic exploration of the experience of searching for meaning in life. Doctoral dissertation, Union Institute.

Hutchfield, K. (1999). Family-centered care: a concept analysis. Journal of Advanced Nursing, 29(5), 1178-1187.

Institute for Family-Centered Care. (n.d.). Advancing the Practice of Patient- and Family-Centered Care How to Get Started. Retrieved on May 17, 2008 from Www.familycenteredcare.org.

Institute for Family-Centered Care. (2008). Partnering with patients and families to design a patient- and family-centered health care system: recommendations and promising practices. Retrieved on August 12, 2008 from http://www.familycenteredcare.org/pdf/PartneringwithPatientsandFamilies.pdf.

Johnson, A. \& Lindschau, A. (1996). Staff attitudes toward parent participation in the care of children who are hospitalized. Pediatric Nursing, 22(2), 99-102. 
Jolley, J. (2007). Separation and psychological trauma: A paradox examined. Pediatric Nursing, 19, 22-25.

Jolley, J., Shield, L. (2009). The evolution of family-centered care. Journal of Pediatric Nursing, 24(2), 164-170.

Jonkees, L. B. (1955). Doctors, hospitals and children. Br J Phys Med, 18, 11-14.

Kerr, L. M. J., Harrison, M. B., Medves, J., Tranmer, J. E., Fitch, M. I. (2007). Understanding the supportive care needs of parents of children with cancer: an approach to local needs assessment. Journal of Pediatric Oncology Nursing, 24(5), 279-293.

Keyser, J. (2006). From parents to partners building a family-centered early childhood program. St. Paul, MN: Redleaf Press.

Klassen, A.F., Dix, D., Cano, S.J., Papsdorf, M., Sung, L., \& Klaassen, R.J. (2008). Evaluating family-centred service in paediatric oncology with the measure of processes of care. Child: Care, health and development, 35(1), 16-22.

Kristensson-Hallstrom, I., \& Elander, G. (1997). Parents' experience of hospitalization: different strategies for feeling secure. Pediatric Nursing, 23, 361-376.

LeGrow, K., \& Rossen, B. E. (2005). Development of professional practice based on family systems nursing framework: nurses and families experience. Journal of Family Nursing, 11(1), 38-58.

Levy, R. I., \& Hollan, D. W. (1998). Chapter ten: person-centered interviewing and observation. In H. R. Bernard. Handbook of methods in cultural anthropology (pp. 333-364). Walnut Creek: Altamira.

Lincoln, Y., \& Guba, E. (1985). Naturalistic Inquiry. New York: Sage. 
Lundqvist, A., \& Nilstun, T. (2007). Human dignity in paediatrics: the effects of health care. Nursing Ethics, 14(2), 215-228.

MacKean, G. L., Thurston, W. E., \& Scott, C. M. (2005). Bridging the divide between families and health professionals' perspectives on family-centered care. Health Expectations, 8, 74-85.

Martin, G. W., \& Younger, D. (2000). Anti oppressive practice: a route to the empowerment of people with dementia through communication and choice. Journal of Psychiatric and Mental Health Nursing, 7, 59-67.

McDonal, V. (1999/2000). A parent's perspective: providing compassionate and effective care. Hospital Quarterly, Winter, 3(2), 20-24.

McGrath (2001). Building relationships with families in the NICU: exploring the guarded alliance. Journal of Perinatal and Neonatal Nursing, 15(3), 74-83.

McKivergin, M. J., \& Daubenmire, M. J. (1994). The healing process of presence. Journal of Holistic Nursing, 12(1), 65-81.

Meleski, D. (2002). Families with chronically ill children: a literature review examines helping them cope. American Journal of Nursing, 102(5), 47-54.

Meuthing, S. E., Kotagal, U. R., Schoettker, P. J., \& del Rey, J.G. (2007). Familycentered bedside rounds: a new approach to patient care and teaching. Pediatrics, 119(4), 829-832.

Miles, M. S. (2003). Support for parents during a child's hospitalization: a nurses guide to helping parents cope. American Journal of Nursing, 103(2), 62-64.

Mitchel, W., Clarke, S., \& Sloper, P. (2006). Care and support needs of children and young people with cancer and their parents. Psycho-Oncology, 15, 805-816. 
Morse, J. (2000). Determining sample size. Qualitative Health Research, 10(1), 3-5.

Moustakas, C. (1994). Phenomenology research methods. Thousand Oaks, CA: Sage.

Muris, P., Steerneman, P., Merckelbach, H., \& Meesters, C. (1996). Parental modelling and fearfulness in middle childhood. Behaviour Research and Therapy, 34 , 265268.

Neuman, W. L. (2006). Social research methods: Qualitative and quantitative approaches. (6th. e.d.). Boston, MA: Pearson.

Ogle, S. K. (2006). Clinical application of family management styles to families of children with cancer. Journal of Pediatric Oncology Nursing, 23(1), 28-31.

Paliadelis, P., Cruickshank, M., Wainohu, D., Winskill, R., \& Stevens, H. (2005). Implementing family-centred care: an exploration of the beliefs and practices of paediatric nurses. Australian Journal of Advanced Nursing, 23(1), 31-36.

Patterson, J. M., \& Hovey, D. L. (2000). Family-centered care for children with special health needs: rhetoric or reality. Families, Systems, and Health, 18, 237-251.

Penkman, L., Scott-Lane, L., \& Pelletier, W. (2006). A psychosocial program for pediatric oncology patients: A pilot study of "the beaded journey." Journal of Psychosocial Oncology, 24(2), 103-115.

Pergert, P. Ekblad, S., Enskar, K., \& Bjork, O. (2007). Obstacles to transcultural caring relationships: experiences of health care staff in pediatric oncology. Journal of Pediatric Oncology Nursing, 24(6), 314-328.

Petersen, M. F., Cohen, J., \& Parsons, V. (2004). Family-centered care: do we practice 
what we preach? Journal of Obstetric, Gynecologic, \& Neonatal Nursing, 33(4), 421-427.

Polit, D., \& Hungler, B. (1997). Essentials of Nursing Research. Philadelphia, PA: Lippincott.

Polit, D. F., \& Hungler, B. P. (1999). Nursing Research Principles and Methods. (6th. ed.). Philadelphia, PA: Lippincott.

Ponte, P. R., Connor, M., DeMarco, R., \& Price, J. (2004). Linking patient and familycentered care and patient safety: the next leap. Nursing Economics, 22(4), 211215.

Pratt, J. D., \& Chitakis, M. (1998). Affirming best practice in pediatric nursing: report to royal children's hospital foundation. Brisbane: Royal Children’s Hospital Foundation.

Public Health Agency of Canada. (1995). This Battle Which I must Fight: Cancer in Canada's Children and Teenagers. Minister of Supply and Services Canada.

Regan, K. M., Curtin, C. C., \& Vorderer, L. (2006). Paradigm shifts in inpatient psychiatric care of children: approaching child- and family-centered care. Journal of Child \& Adolescent Psychiatric Nursing, 19(1), 29-40.

Ryan, E., \& Steinmiller, E. (2004). Modeling family-centered pediatric nursing care: strategies for shift report. Journal for Specialists in Pediatric Nursing, 9(4), 123134.

Schwartz, K. (1995, July 16). A patient's story. Boston Globe, pp. 15-20, 23-27.

Scott, L. (1998). Perceived needs of parents of critically ill children. Journal of the Society of Pediatric Nurses, 3(1), 4-11. 
Shelton, T. L., \& Stepanek, J. S., (1994). Family-centered care for children needing specialized health and developmental services. (3rd. Ed.). Bethesda, MA: Association for the Care of Children's Health.

Shields, L. (2007). Family-centered care in the perioperative area: an international perspective. ARON Journal, 85(5), 893-902.

Shields, L., Pratt, J., Davis, L., \& Hunter, J. (2007). Family-centered care for children in hospital. Cochrane Database of Systematic Reviews. Issue 1. Art. No.: CD004811. DOI: 10.1002/14651858.CD004811.pub2.

Shields, L., Pratt, J., \& Hunter, J. (2006). Family-centered care: a review of qualitative studies. Journal of Clinical Nursing, 15(10), 1317-1323.

Shields, L. \& Tanner, A. (2004). Pilot study of a tool to investigate perceptions of familycentered care in different settings. Pediatric Nursing, 30(3), 189-197.

Sobo, E. J. (2004). Good communication in pediatric cancer care: a culturally-informed research agenda. Journal of Pediatric Oncology Nursing, 21(3), 150-154.

Streubert, H. J., \& Carpenter, D. R. (1995). Qualitative Research in Nursing (2nd ed.). Philadelphia, PA: Lippincott Williams and Wilkins.

Titone, J., Cross, R., Sileo, M., Martine, G. (2004). Taking family-centered care to a higher level on the heart and kidney unit. Pediatric Nursing, 30(6), 495-497.

Tri-Council Policy Statement: Ethical Conduct for Research Involving Humans. (2005). Canadian Insitutes of Health Research, Natural Sciences and Engineering Research Council of Canada, Social Sciences and Humanities Research Council of Canada. Public Works and Government Services of Canada.

Tomlinson, D. (2004). Paediatric oncology nurse education: the development of a 
national framework. Journal of Clinical Nursing, 13, 646-654.

Trask, P. C., Paterson, A. G., Trask, C. L., Bares, C. B., Birt, J., \& Maan, C. (2003). Parents and adolescent adjustment to pediatric cancer: associations with coping, social support, and family function. Journal of Pediatric Oncology Nursing, 20(1), 36-47.

Turner, M., Tomlinson, P., Harbaugh, B., \& Anderson, K. (1996). The influence of illness severity and family resources on maternal uncertainty during critical pediatric hospitalization. American Journal of Critical Care Nursing, 5(2), 140146.

Van Riper, M. (2001). Family-provider relationships and well-being in families with preterm infants in the NICU. Heart \& Lung: The Journal of Acute \& Critical Care, 30(1), 74-84.

Vincent, J., Alexander, B., \& Patterson, M. (1996). How parents describe caring behaviors of nurses in pediatric intensive care. Maternal Child Nursing, 21, 197201.

Ward-Smith, P., Kirk, S., Hetherington, M., \& Hubble, C. L. (2005). Having a child diagnosed with cancer: an assessment of values from the mother's viewpoint. Journal of Pediatric Oncology Nursing, 22(6), 320-327.

Widger, K. A., \& Wilkins, K. (2004). What are the key components of quality of perinatal and pediatric end-of-life care? a literature review. Journal of Palliative Care, 20(2), 105-112.

Wilkins, K. L., \& Woodgate, R. L. (2005). A review of qualitative research on the 
childhood cancer experience from the perspective of siblings: a need to give them a voice. Journal of Pediatric Oncology Nursing, 22(6), 305-319.

Wolf, L. (2004). Should parents speak with a dying child about impending death? The New England Journal of Medicine. 351, 1251-1253.

Woodgate, R. L. (2006). The importance of being there: perspectives of social support by adolescents with cancer. Journal of Pediatric Oncology Nursing, 123(3), 122-134.

Woodgate, R. L., \& Degner, L. F. (2003). A substantive theory of keeping the spirit alive: the spirit withing children with cancer and their families. Journal of Pediatric Oncology Nursing, 20(3), 103-119.

Yingling, J. (2000). Verbal responses of children and their supportive providers in a pediatric oncology unit. Journal of Health Communications, 5(2), 371-377. 
Appendix A

Poster

Exploring Family-Centered Care Among Pediatric Oncology

Nurses

Information Pamphlets Below: Please Help Yourself! 
Appendix B

Information Sheet (originally in a pamphlet format)

\section{Exploring Family-Centered Care among Pediatric Oncology Nurses}

\section{A Qualitative Study}

Family-centered care is an important aspect of practice among pediatric oncology nurses. However, given the increased demands placed on nurses today, (eg., staff shortages, increased patient load, acuity of patients, increased technology etc.) nurses are challenged to provide family-centered care.

The purpose of this study is to explore pediatric oncology nurses perspectives and experiences in providing family-centered care in light of such challenges. 
Inclusion Criteria: Staff Registered Nurses and/or Registered Nurse Practitioners who work in the oncology in-patient unit or oncology out-patient clinic at the Alberta Children's Hospital.

Should you decide to Participate:

- You will be involved in a one-hour interview at a location of your choosing.

- You will be given the chance to voice and share your experiences about FamilyCentered Care.

- You will be given the opportunity to discuss the extent to which you provide Family-Centered Care.

- You will be able to express and describe the barriers that you feel exist while trying to provide care for patients and their families that is family-centered.

- You will receive a $\$ 5.00$ gift certificate (to Good Earth Cafe) at the end of your interview.

If you are interested in participating in this study, or if you would like further information please contact Lyndsay MacKay. phone: (403) 955-7827 e-mail: lyndsay.mackay@albertahealthservices.ca

- Graduate Student: School of Health Sciences at the University of Lethbridge

- $\quad$ Supervisor: Dr. David Gregory, (403) 329-2432: david.gregory@uleth.ca 
Appendix C

\section{Alberta Health Services}

\section{Alberta Children's Hospital}

\section{Participant Information and Consent Form for Participation in Research}

INFORMATION AND CONSENT FORM FOR PARTICIPATION IN RESEARCH

Study Title:

Exploring Pediatric Oncology Nurses’ Perspectives about Family Centered Care (FCC)

\section{Local Principal Investigator:}

Local Principle Investigator: Dr. Aru Narendaren

Local Co-Investigator: Lyndsay Jerusha MacKay, RN, BN

Other Co-Investigator: Dr. David Gregory

Sponsor: $\quad$ Child Health Research Office at the Alberta Children's Hospital

\section{INTRODUCTION:}

I am asking you to volunteer to participate in a qualitative research study. Before you can decide whether or not to volunteer for this study, you must understand its purpose, how it may affect you, the risks involved, and what is expected of you should you decide to participate. This process is called informed consent.

I am asking you to take part in this qualitative research study because you are a pediatric oncology nurse and work in the Oncology Department at the Alberta Children's Hospital $(\mathrm{ACH})$.

\section{PURPOSE OF THE STUDY:}

The purpose of this study is to explore the perspectives and experiences of familycentered care among pediatric oncology nurses in light of current barriers and challenges. I want to discover the practical skills and activities that you use to provide familycentered care. I want to understand what barriers and challenges exist that hinder you from providing family-centered care. I would like to gain an understanding of your 
experiences in providing FCC. Findings from this study may be used to create in-service education sessions, presentations and education days. As well, the findings may be published in journals and/or presented at public conferences.

\section{NUMBER OF PARTICIPANTS:}

Up to 20 nurses, both registered nurses and registered nurse practitioners will partake in this study at the Alberta Children's Hospital.

\section{DURATION OF PARTICIPATION:}

Although the overall study will take one year to complete, your time commitment is a one-time, one-hour interview.

\section{PROCEDURES:}

The research interview will occur during a time and at a location that is conveniently arranged between the Principle Investigator and yourself. You will be given a gift certificate of appreciation to Good Earth Café, a value of $\$ 5.00$ for taking time out of your schedule to participate.

Basic demographic information about you (for example, your gender, years practicing as a pediatric oncology nurse, etc.) will be collected at the beginning of the interview. You will then be interviewed for approximately one hour. You will be asked various questions about family-centered care and your experiences in providing such care to patients and their families. Also, you will be asked about the barriers and challenges that prevent you from providing family-centered care. The interview will be digitally recorded and transcribed verbatim (word for word) by a professional transcriptionist who will sign an oath of confidentiality in relation to your interview.

\section{RISKS AND SIDE EFFECTS:}

The interview may possibly explore sensitive topics regarding your experiences providing care to pediatric patients with cancer and their families. If you feel you need to discuss these issues further you contact the Employee Family Assistance Program (EFAP) at 1-800-268-5211 to set up a counseling time. If you experience any negative effects, you will not be compensated for such in any way.

\section{POTENTIAL BENEFITS:}

Participating in this study may benefit patients and their families, because a better understanding of family-centered care will be gained and shared with the nurses in the Oncology Department. The nurses will benefit from this study because barriers, frustrations and challenges to providing family-centered care will be identified. The findings may be shared with nurses through publications, educations sessions, in-services and presentations. In the future it is anticipated that activities and actions can be put into 
place to address such barriers and challenges. An executive summary will be shared with you, should this be of interest to you.

\section{VOLUNTARY PARTICIPATION AND WITHDRAWAL:}

Taking part in this study is completely voluntary. You have the right to choose not to be in this study. Your work and/or career in the Oncology Department will not be jeopardized in any way by choosing not to participate or should you decide to withdraw from the study. You can stop your participation in this research study at any time after giving your consent without any negative consequences.

\section{COMPENSATION AND COSTS:}

There is no cost to you for participation. You will receive a \$5.00 gift certificate to Good Earth Cafe for participating in this study whether or not you fully complete the interview.

\section{NEW FINDINGS:}

I will learn new information during this study regarding the nature in which pediatric oncology nurses provide FCC and the challenges and barriers to implementing FCC into nursing practice. These findings will be shared with the participants through an executive summary of the findings that will be provided to you.

\section{CONFIDENTIALITY:}

I will keep the information about you private. To protect your privacy, you will not be identifiable in any study reports; your identification will be kept anonymous to the best of my ability. However, there is potential risk that a small sample of participants in the same institution may know each other and share conversation about being in the study. Your interview and demographic data will be coded with a number so that no personal information or names are present. The data and personal information, including a list of code numbers and names, will be locked in a filing cabinet separate from the interview/demographic data. Only the project researcher and supervisor will have access to the anonymized interview information. Within 7 years after completion of the study, the transcripts will be destroyed completely by shedding the paper, and the audio/digital record will be erased/deleted. All destroyed materials will be disposed of as confidential waste. The transcriber of the transcripts will sign an oath of confidentiality, stating that he/she will not share or talk about the information in the interview with anyone besides Lyndsay MacKay.

\section{SIGNATURES:}

Your signature on this form indicates that you have understood to your satisfaction the information regarding your participation in the research project and agree to participate as a subject. In no way does this waive your legal rights nor release the investigators, or 
involved institutions from their legal responsibilities. You are free to withdraw from the study at any time without any negative consequences. If you have further questions concerning matters related to this research, please contact:

\section{Lyndsay MacKay (403) 955 7827; Lyndsay.Mackay@calgaryhealthregion.ca}

\section{Dr. David Gregory, Graduate Supervisor, (403) 329 2432; David.Gregory@uleth.ca}

If you have any further questions concerning your rights as a possible participant in this research, please contact the Ethics Resource Officer, Internal Awards, Research Services, University of Calgary at (403) 2207990 or the Human Subject Research Coordinator, Research Services, University of Lethbridge, (403) 329-2747.

Participant's Name

Investigator Name
Signature and Date

Signature and Date

By signing below you give Lyndsay MacKay to have the transcripts transcribed verbatim:

Participant's Name
Signature and Date

The University of Calgary Conjoint Health Research Ethics Board and the Research Services at the University of Lethbridge has approved this research study.

A signed copy of this consent form will be given to you to keep for your records and reference.

If you wish to receive an executive summary to you, please fill in the following:

Participant's Name:

Participant's Address: 
Appendix D

Demographic Questionnaire

\section{Participant Demographic Information}

Date:

Name:

Pseudonym:

Phone Number:

E-Mail:

Gender:

Male

Female

Age:

Education:

Nursing Diploma

Nursing Degree

Master's of Nursing/Nurse Practitioner

Other

Number of years working as a nurse:

Number of years working in pediatric oncology:

Completed a course in Family-Centered Care/Family Nursing: Yes / No: if yes, when:

In-patient Nurse / Out-patient Nurse

Registered Nurse / Registered Nurse Practitioner 


\section{Appendix E}

Interview Guide

The Institute for Family-Centered Care has developed four core concepts of familycentered care. They are retrieved from http://www.familycenteredcare.org/faq.html. They will be used to provide guidance to the interview as follows. The participants will be asked to give an example of each.

\section{Opening Questions:}

What does FCC mean to you?

Can you describe one experience in which you feel you provided care that was family-centered?

Can you describe one experience in which you where unable to provide FCC? What happened?

\section{Institutional Support:}

- Do you think the ACH supports FCC in your setting? How so?

- What has ACH done to support and encourage FCC in your setting?

- Do you think that $\mathrm{ACH}$ provides educational opportunities and learning sessions for FCC? Please describe.

- What barriers and challenges are present in your work setting that might hinder you from providing FCC?

- Tasks overtake focus from FCC? Too busy? When busy, experience job satisfaction? Job satisfaction increase FCC?

\section{Dignity and Respect:}

- Can you give me an example of how you successfully listened to a patient or family member which resulted in a positive experience for yourself and the family? Or did not listen?

- How do you honor and respect your patient and their family perspectives? Can you give an example? When is it not possible to do so? Can you give me an example?

- How do you allow your patients and their family to make choices? Can you give an example? When is it not possible to do so? Can you give me an example?

- How do you incorporate the patients and families knowledge, values, beliefs, and cultural background into the planning and delivery of care? Can you give an example when this worked? Can you give me an example when it did not work?

- Can you provide me with an example when you cared for a family with a different culture than your own. Describe what worked and was successful and what did not work and was hindering to the caring process.

\section{Information Sharing:}


- Is it possible to communicate and share complete and unbiased information with patients and their families? Can you provide an example?

- How do you communicate such information in ways that are sensitive, affirming and useful?

- How do you provide information in a timely manner?

- How do you provide information to patients so that they can effectively participate in care and decision making?

\section{Participation:}

- How do you support and encourage patients to participate in care and decision making?

- How do you support and encourage patients to choose the level of participation in their care?

- Can you provide me with an example of when this worked well? Why?

- Can you provide me with an example when this did not work well? Why?

\section{Collaboration:}

- Do you feel it is important to collaborate with patients and their families?

- How do you include patients and families in development of care plans and delivery of care for patients?

- How do you include patients and families in the evaluation of care? 


\section{Appendix F}

\section{Oath of Confidentiality}

In participating in this research project, "Exploring Pediatric Oncology Nurses' Perspectives about Family Centered Care (FCC)" I agree to treat information gained from this study in a confidential manner. I will neither identify, nor will I discuss with anyone, other than Lyndsay Jerusha MacKay, the contents of the interviews.

Name

Date

Signature 


\section{Appendix G}

Timeline and Budget

Table 1

Anticipated Timeline

\begin{tabular}{|l|l|l|}
\hline \multicolumn{1}{|c|}{ Required Tasks } & \multicolumn{1}{|c|}{ Task Objectives } & \multicolumn{1}{c|}{ Anticipated Timeline } \\
\hline Literature Review & $\begin{array}{l}\text { Identify gaps in literature } \\
\text { Summarize literature that acts as basis for } \\
\text { this study }\end{array}$ & Fall 2007 - Summer 2008 \\
\hline $\begin{array}{l}\text { Thesis Proposal } \\
\text { Development }\end{array}$ & $\begin{array}{l}\text { Develop accurate, concise and feasible } \\
\text { proposal with supervisor and other } \\
\text { committee members }\end{array}$ & Summer 2008 - Fall 2008 \\
\hline Ethics Approval & Submit proposal for ethics review & Fall 2008 \\
\hline Participant Identification & $\begin{array}{l}\text { Contact year coordinators, instructors and } \\
\text { students } \\
\text { Schedule data collection procedures }\end{array}$ & Fall 2008 - Winter 2008 \\
\hline Data Collection & Individual interviews of at least 20 nurses & Winter 2008 - Winter 2009 \\
\hline Data Analysis & $\begin{array}{l}\text { Thematic analysis of all data } \\
\text { Interpretations \& Recommendations }\end{array}$ & Spring 2009- Summer 2009 \\
\hline Thesis Writing and Editing & $\begin{array}{l}\text { All chapters complete and edited version } \\
\text { ready for submission }\end{array}$ & $\begin{array}{l}\text { Summer 2009 - Fall 2009 } \\
\text { (First three chapters to be } \\
\text { done by Fall 2007) }\end{array}$ \\
\hline Thesis Defense & Prepared for thesis defense & Fall 2009 \\
\hline
\end{tabular}

Table 2

Anticipated Budget

\begin{tabular}{|l|l|c|}
\hline \multicolumn{1}{|c|}{ Task } & \multicolumn{1}{c|}{ Item cost } & Total Cost \\
\hline Printing and Materials & Printing \$150, Ink \$150 & $\$ 300$ \\
\hline Transcription costs & $201.5 \mathrm{hr}$ transcripts*3hr transcribing*\$20/hr & $\$ 1200$ \\
\hline & \multicolumn{1}{c|}{ Grand Total } & $\$ 1500$ \\
\hline
\end{tabular}

